\title{
Array Processing and Forward Modeling Methods for the Analysis of Stiffened, Fluid-Loaded Cylindrical Shells
}

by

Joseph E. Bondaryk

M.S., Electrical Engineering, M.I.T (1988)

B.S., Electrical Engineering and Computer Science, M.I.T (1987)

Submitted in partial fulfillment of the requirements for the degree of

DOCTOR OF PHILOSOPHY IN OCEANOGRAPHIC ENGINEERING

at the

\section{MASSACHUSETTS INSTITUTE OF TECHNOLOGY}

and the

\section{WOODS HOLE OCEANOGRAPHIC INSTITUTION}

March 1994

(C) Massachusetts Institute of Technology and Woods Hole Oceanographic Institution, 1994

All rights reserved.

Signature of Author

Joint Program in Applied Ocean Science and Engineering Massachusetts Institute of Technology

Woods Hole Oceanographic Institution

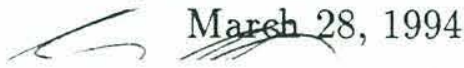

Certified by

Dr. Henrik Schmidt

Professor, Massachusetts Institute of Technology $\bigcap$. Thesis Supervisor

Accepted by

Dr. Arthur BJ Baggeroer

Chairman, Joint Committee for Apratiode Ocean Science and Engineering Massachusetts Institute of Technology $y_{8}$ Woo Hớ Oceanographic Institution 


\title{
Array Processing and Forward Modeling Methods for the
}

\section{Analysis of Stiffened, Fluid-Loaded Cylindrical Shells}

\author{
by \\ Joseph E. Bondaryk \\ Submitted to the Massachusetts Institute of Technology/
Woods Hole Oceanographic Institution
Joint Program in Applied Ocean Science and Engineering
on March 28, 1994, in partial fulfillment of the
requirements for the degree of
Doctor of Philosopy
}

\begin{abstract}
This thesis investigates array processing and forward modeling methods for the analysis of experimental, structural acoustic data to understand wave propagation on fluid-loaded, elastic, cylindrical shells in the mid-frequency range, $2<k a<12$. The transient, acoustic, in-plane, bistatic scattering response to wideband, plane waves at various angles of incidence was collected by a synthetic array for three shells, a finite, air-filled, empty thin shell, a duplicate shell stiffened with four unequally spaced ring-stiffeners and a duplicate ribbed shell augmented by resiliently-mounted, wave-bearing, internal structural elements.
\end{abstract}

Array and signal processing techniques, including source deconvolution, array weighting, conventional focusing and the removal of the geometrically scattered contribution, are used to transform the collected data to a more easily interpreted representation. The resulting waveforms show that part of the transient, dynamic, structural response of the shell surface which is capable of radiating to the far field. Compressional membrane waves are directly observable in this representation and evidence of flexural membrane waves is present. Comparisons between the shells show energy compartmentalized by the ring stiffeners and coupled into the wave-bearing internals. Energy calculations show a decay rate of $30 \mathrm{~dB} / \mathrm{msec}$ due to radiation for the Empty shell but only $10 \mathrm{~dB} / \mathrm{msec}$ for the other shells at bow incidence. The Radon Transform is used to estimate the reflection coefficient of compressional waves at the shell endcap as 0.2 .

The measurement array does not provide enough resolution to allow use of this technique to determine the reflection, transmission and coupling coefficients at the ring stiffeners. Therefore, a forward modeling technique is used to further analyze the $0^{\circ}$ incidence case. This modeling couples a Transmission Line model of the shell with a Simulated Annealing approach to multi-dimensional, parameter estimation. This procedure estimates the compressional wavespeed at $5284 \mathrm{~m} / \mathrm{sec}$ and a compressional decay rate of $49 \mathrm{~dB} / \mathrm{msec}$. Small cross-coupling coefficients between flexural and 
compressional wavetypes at the slope discontinuities on the Empty shell are found to be responsible for most of the radiation later in time. High reflection coefficients at the ring stiffeners on the Ribbed shell are shown to cause energy compartmentalization in the bays between ribs and pressure doubling of incident structural waves at the ribs.

Thesis Supervisor: Dr. Henrik Schmidt

Professor, Massachusetts Institute of Technology 


\section{Acknowledgments}

A thesis like this one is the work of a community supporting one man's individual efforts. By sharing in his failures, the community has earned the right to share in his success. Let him never forget those who made it possible and fool himself into thinking he did it alone.

First and foremost, I would like to thank my advisor, Prof. Henrik Schmidt. You've always been there when I needed you and not there when I didn't need you; this alone made you the perfect advisor for me. A great sense of humor and an incredibly sharp technical sense are just two of your superb qualities. It was a great privilege working for you; I look forward now to working with you.

I also owe a great debt of gratitude to the entire acoustics group for their constant inspiration. Thanks to my thesis committee, Rob Fricke, Josko Catipovic and Yueping Guo, for all your useful comments throughout this lengthy process and for generally keeping me thinking. It is my eventual goal to be a professor somewhere, it is my hope that someday I will be able to teach someone as well as Henrik, Ira Dyer and Art Baggeroer have taught me.

Thanks to the entire acoustics group administrative staff. Sabina, Taci, Isela, Mary, Denise and Marilyn, thank you all for everything, you do an impossible job keeping us all happy.

Support for this thesis was provided by Office of Naval Research, Structural Mechanics and Advanced Vehicle Technology Divisions, it is most gratefully acknowledged. Thanks to John Spanier and Brian Houston at NRL for doing a great job getting us this terrific data.

Without my office-mates I never would have made it through. Special thanks to Matt Conti for singing the Slinky song with me at 2AM many a night, Tarun Kapoor for scaring away all the quiet people, 2nd order Dave Ricks for having too much coffee 
and Chick Corrado for every half-empty cup in the place. Remember, we've played too many dart games together to ever forget one another. Thanks to Dan McCarthy, Brian Sperry, Kevin Lapage and the rest of the 5-435 and 5-007 crew for all the discussions both technical and silly. And who could leave out...Gopal.

I will always fondly recall my summers spent at WHOI, they were all too short. Thanks to all the staff that made it fun, Abbie, Jake, Jim Lynch and especially M.J. Kudos to SEA for a great cruise and the Food Buoy for the great muffins. For all the windsurfing, to the members of Team Bondaryk, Jaime, Hanumant and Fred, "until the wind is up." Special thanks to "Honest" Al Oppenheim, windsurfing salesman extraordinaire. I'll particularly miss those late night beers at the Captain Kidd with Van Gurley and Chris Howell and I hope the wind will again be strong enough for a Single Malt with my old office-mate John Buck.

Thanks to all the gang at Atlantic Aerospace Electronics Corp. for all the fun, deep thought and profit I got out of consulting these many years. I'm particularly grateful to Ted, Victor, Eric, Tami and Sven for the work, I learned as much from you as I did in school. Special thanks to Tony my UNIX answerman and to Paul "\#26 and hold the onion" Baim. Thanks also to Eli Peli at the Schepens Eye Research Institute for showing me what it means to see.

Thanks to all my Masters for giving me the tools to stay sane and enjoy life during this long technical road: Stephan Driscoll my dance master accompanied by Mary Dockham, Michael McLauglin my piano master, Alan Brody my playwriting master and the entire MIT theatrical community.

Ma, I'm done, I promise to be less of a grouch now. Thanks for the donuts, TV guide and all the family news on Sunday mornings for the last 5 years. John, Zita, Bob, Nancy, you're what family should be, supportive and loving, thank you. To all my kids, Melissa, Elizebeth, Michael, Matthew, Susan and Samantha, thanks for being around for me to love. Grow up smart, strong and happy. And to my new family, Julien and Susan, thank you for all the love and support you've given your daughter and now me, I look forward to many fun years ahead with you. 
Finally, for sticking with me through the "dark times," for all the letters, phone calls, flights, drives and e-mail, for all the little things, for being a thesis widow these last few months, for being able to talk with me about absolutely anything, for making me happy every minute of every day, I love you Leslie. I can't wait to start our married life together. 


\section{Dedication}

To my Shmuffin

Leslie 


\section{Contents}

1 Introduction $\quad 16$

1.1 Motivation ......................... 16

1.2 Previous Research . . . . . . . . . . . . . . . 18

1.3 Hybrid Processing Approach . . . . . . . . . . . . . . . . . 20

1.4 Organization of Thesis ..................... 23

2 Array Processing $\quad 26$

2.1 Motivation .......................... 26

2.1.1 Model Designs. . . . . . . . . . . . . . 28

2.1 .2 Experiment . . . . . . . . . . . . . . 30

2.2 Conventional Beamforming . . . . . . . . . . . . 31

2.3 Conventional Focusing . . . . . . . . . . . . . . 35

2.4 MLM Focusing . . . . . . . . . . . . . . . . 38

2.5 Radon Transform . . . . . . . . . . . . . . . . . . 40

3 Array Processing Results $\quad 42$

3.1 Raw Experimental Data . . . . . . . . . . . . . 43

3.1.1 Full Dataset . . . . . . . . . . . . . . . . 43

3.1 .260 Degree Subarray . . . . . . . . . . . . 51

3.2 Deconvolution with Source Pulse . . . . . . . . . . . . . 60

3.2.1 Process .............................. 60

3.2.2 Results....................... 61

3.3 Conventional Focusing Results . . . . . . . . . . . . . . 64 
3.3.1 Rectangular array weighting . . . . . . . . . . . 64

3.3.2 Hamming tapered array weighting . . . . . . . . . . 64

3.4 Removal of Geometrically Scattered Return _ . . . . . . . . . 69

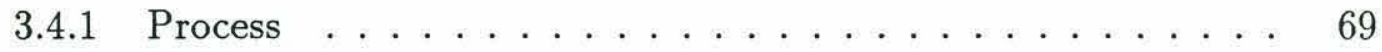

3.4 .2 Results . . . . . . . . . . . . . . . . 70

3.5 Results of Processing Chain for All Data . . . . . . . . . . . 73

3.6 Energy . . . . . . . . . . . . . . . . . . . . 84

3.7 Resolution of Array Processing _. . . . . . . . . . . . . . 91

3.7.1 Results of MLM Beamforming . . . . . . . . . . . . 93

3.7 .2 Cramer-Rao Bounds . . . . . . . . . . . . . . 95

3.8 Radon Transform Processing . . . . . . . . . . . . . . . . 98

4 Forward Modeling $\quad 106$

4.1 Motivation . . . . . . . . . . . . . . . . . 106

4.2 Transmission Line Model . . . . . . . . . . . . . . . . . . . 109

4.2 .1 Motivation . . . . . . . . . . . . . . . . 109

4.2 .2 Model details . . . . . . . . . . . . . . . . . . . 111

4.3 Parameter Estimation $\ldots \ldots \ldots \ldots$

5 Forward Modeling Results $\quad 125$

5.1 Compressional Waves on the Empty Shell . . . . . . . . . . . 126

5.2 Flexural Waves on the Endcaps . . . . . . . . . . . . . . 132

5.3 Flexural Waves on the Empty Shell . . . . . . . . . . . . . . 135

5.4 Ring Stiffeners on the Ribbed Shell . . . . . . . . . . . . . . . 139

6 Conclusion $\quad 145$

6.1 Discussion of Results . . . . . . . . . . . . . . . . . 145

6.2 Suggestions for Further Research . . . . . . . . . . . . . . . 148

A T-line Program Input File $\quad 150$ 


\section{List of Figures}

1-1 Direct analysis approach. . . . . . . . . . . . . 20

1-2 Forward modeling approach . . . . . . . . . . . . . 21

1-3 Hybrid approach . . . . . . . . . . . . . 23

2-1 Empty shell design. . . . . . . . . . . . . . . . . . . 28

$2-2$ Ribbed shell design. . . . . . . . . . . . . . . . . . . . . 29

2-3 Ring and suspended mass system in the Complex shell. . . . . . . . 29

2-4 Wave-bearing system in the Complex shell. . . . . . . . . . . 30

2-5 Experimental geometry. . . . . . . . . . . . . . . 31

$2-6$ General beamforming structure . . . . . . . . . . . . 33

2-7 Intuitive focusing operation $\ldots \ldots \ldots \ldots \ldots \ldots \ldots$

2-8 Synthetic focusing example . . . . . . . . . . . . . . 37

2-9 Radon Transform example . . . . . . . . . . . . . . . . 41

3-1 Raw data from the Complex shell. Incident angle $=90^{\circ}$. Receivers

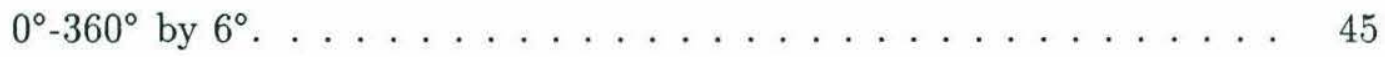

3-2 Raw data from the Complex shell. Incident angle $=90^{\circ}$. Receivers $0^{\circ}-360^{\circ}$ by $6^{\circ}$. Each line scaled $\min$ to $\max . \ldots \ldots \ldots$

3-3 Raw data from the Empty shell. Incident angle $=75^{\circ}$. Receivers $0^{\circ}$ $360^{\circ}$ by $6^{\circ}$. Each line scaled $\min$ to $\max . \ldots \ldots \ldots \ldots$

3-4 Raw data from the Ribbed shell. Incident angle $=75^{\circ}$. Receivers $-105^{\circ}-75^{\circ}$ by $3^{\circ}$. Each line scaled $\min$ to $\max . \ldots \ldots \ldots$

3-5 Raw data from the Complex shell. Incident angle $=75^{\circ}$. Receivers $0^{\circ}-360^{\circ}$ by $6^{\circ}$. Each line scaled min to max. 
3-6 Raw data from the Complex shell. Incident angle $=66^{\circ}$. Receivers $0^{\circ}-360^{\circ}$ by $6^{\circ}$. Each line scaled $\min$ to $\max . \ldots \ldots \ldots \ldots$

3-7 Raw data from the Empty shell. Incident angle $=0^{\circ}$. Receivers $0^{\circ}-180^{\circ}$ by $3^{\circ}$. Each line scaled $\min$ to $\max \ldots \ldots \ldots \ldots \ldots \ldots$

3-8 Raw data from the Ribbed shell. Incident angle $=0^{\circ}$. Receivers $0^{\circ}$ $180^{\circ}$ by $3^{\circ}$. Each line scaled $\min$ to $\max . \ldots \ldots \ldots \ldots$

3-9 Raw data from the Complex shell. Incident angle $=0^{\circ}$. Receivers $0^{\circ}-180^{\circ}$ by $3^{\circ}$. Each line scaled $\min$ to $\max . \ldots \ldots \ldots$

3-10 Raw data from the Empty shell. Incident angle $=5^{\circ}$. Receivers $0^{\circ}-360^{\circ}$ by $6^{\circ}$. Each line scaled $\min$ to $\max . \ldots \ldots \ldots \ldots$

3-11 Raw data from the Complex shell. Incident angle $=5^{\circ}$. Receivers $0^{\circ}-360^{\circ}$ by $6^{\circ}$. Each line scaled $\min$ to $\max . \ldots \ldots \ldots$

3-12 Raw data from $60^{\circ}$ of the array by $1^{\circ}$ around shell beam. Empty shell

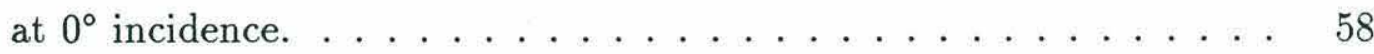

3-13 Raw data from $60^{\circ}$ of the array by $1^{\circ}$ around shell beam. Ribbed shell

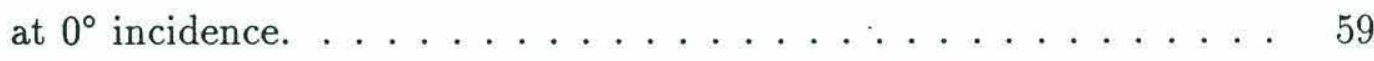

3-14 (a) Source pulse and (b) Source spectrum. . . . . . . . . . . 60

3-15 (a) Hamming source pulse and (b) Hamming source spectrum. . . . . 61

3-16 Raw data from $60^{\circ}$ of the array by $1^{\circ}$ around shell beam, deconvolved by source pulse. Empty shell at $0^{\circ}$ incidence. . . . . . . . . . 62

3-17 Raw data from $60^{\circ}$ of the array by $1^{\circ}$ around shell beam, deconvolved by source pulse. Ribbed shell at $0^{\circ}$ incidence. . . . . . . . . 63

3-18 $60^{\circ}$ of array around shell beam, deconvolved by the source pulse and focused onto shell. Empty shell data at $0^{\circ}$ incidence. . . . . . . . 65

3-19 $60^{\circ}$ of array around shell beam, deconvolved by the source pulse and focused onto shell. Ribbed shell data at $0^{\circ}$ incidence. . . . . . . . 66

3-20 $60^{\circ}$ of array around shell beam, deconvolved by the source pulse, Hamming tapered and focused onto shell. Empty shell data at $0^{\circ}$ incidence. 67

3-21 $60^{\circ}$ of array around shell beam, deconvolved by the source pulse, Hamming tapered and focused onto shell. Ribbed shell data at $0^{\circ}$ incidence. 68 
3-22 Block diagram of process used to remove geometrically scattered return. 69

3-23 Amplitude estimate for the geometrically scattered return from the Empty (bold), Ribbed (plain) and Complex (dotted) shells at $0^{\circ}$ inci-

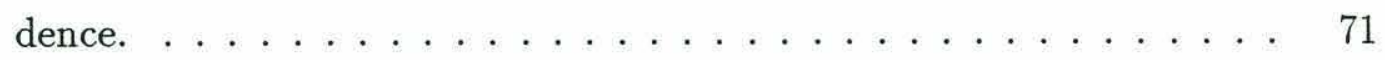

3-24 Amplitude estimate for the geometrically scattered return from the Empty (bold), Ribbed (plain) and Complex (dotted) shells at $75^{\circ}$ incidence. . . . . . . . . . . . . . . . . . . 72

3-25 Block diagram of complete direct analysis processing chain. . . . . . 73

3-26 $60^{\circ}$ of array around shell beam, geometrically scattered return removed, deconvolved by the source pulse, Hamming tapered and focused onto shell. Empty shell data at $0^{\circ}$ incidence. . . . . . . . . . . . 74

3-27 $60^{\circ}$ of array around shell beam, geometrically scattered return removed, deconvolved by the source pulse, Hamming tapered and focused onto shell. Ribbed shell data at $0^{\circ}$ incidence. . . . . . . . . . 75

3-28 $60^{\circ}$ of array around shell beam, geometrically scattered return removed, deconvolved by the source pulse, Hamming tapered and focused onto shell. Complex shell data at $0^{\circ}$ incidence. . . . . . . . . . . . 76

3-29 $60^{\circ}$ of array around shell beam, geometrically scattered return removed, deconvolved by the source pulse, Hamming tapered and focused onto shell. Empty shell data at $5^{\circ}$ incidence. . . . . . . . . . . . . .

3-30 $60^{\circ}$ of array around shell beam, geometrically scattered return removed, deconvolved by the source pulse, Hamming tapered and focused onto shell. Complex shell data at $5^{\circ}$ incidence. . . . . . . . . . . . . .

3-31 $60^{\circ}$ of array around shell beam, geometrically scattered return removed, deconvolved by the source pulse, Hamming tapered and focused onto shell. Empty shell data at $75^{\circ}$ incidence. . . . . . . . . . . . 80

3-32 $60^{\circ}$ of array around shell beam, geometrically scattered return removed, deconvolved by the source pulse, Hamming tapered and focused onto shell. Ribbed shell data at $75^{\circ}$ incidence. . . . . . . . . . . . 
3-33 $60^{\circ}$ of array around shell beam, geometrically scattered return removed, deconvolved by the source pulse, Hamming tapered and focused onto shell. Complex shell data at $75^{\circ}$ incidence. . . . . . . . . . . . 82

3-34 $60^{\circ}$ of array around shell beam, geometrically scattered return removed, deconvolved by the source pulse, Hamming tapered and focused onto shell. Complex shell data at $66^{\circ}$ incidence. . . . . . . . . . . 83

3-35 $60^{\circ}$ of array around shell beam, geometrically scattered return removed, deconvolved by the source pulse, Hamming tapered and focused onto shell. Complex shell data at $90^{\circ}$ incidence. . . . . . . . . . . . . .

3-36 Response of shell surface, energy integrated over space. Empty (bold), Ribbed (plain) and Complex (dotted) shells for $0^{\circ}$ incidence case. . . 87

3-37 Response of shell surface, energy integrated over time. Empty (bold), Ribbed (plain) and Complex (dotted) shells for $0^{\circ}$ incidence case. . . 88

3-38 Response of shell surface, energy integrated over space. Empty (bold), Ribbed (plain) and Complex (dotted) shells for $75^{\circ}$ incidence case. . . 89

3-39 Response of shell surface, energy integrated over time. Empty (bold), Ribbed (plain) and Complex (dotted) shells for $75^{\circ}$ incidence case. . . 90

3-40 Response of shell surface, energy integrated over space. Complex shell at $66^{\circ}$ incidence (bold) and Complex shell at $75^{\circ}$ incidence (plain). . .

3-41 Response of shell surface, energy integrated over time. Complex shell at $66^{\circ}$ incidence (bold) and Complex shell at $75^{\circ}$ incidence (plain). . .

3-42 $60^{\circ}$ of array around shell beam, geometrically scattered return removed, deconvolved by the source pulse, and MLM focused onto shell. Ribbed shell data at $0^{\circ}$ incidence. . . . . . . . . . . . . . . . . . 93

3-43 Response of shell surface, energy integrated over time. Ribbed shell for $0^{\circ}$ incidence case, conventional (bold) and MLM focusing (plain).

3-44 Cramer-Rao bound estimate of resolution of source in presence of sen-

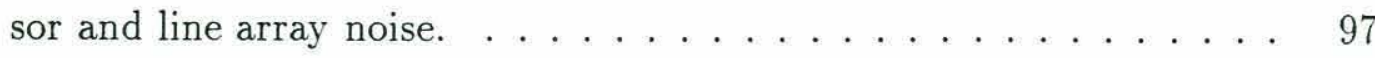

3-45 Radon transform of waves traveling on the Empty shell using $0^{\circ}$ incidence data. . . . . . . . . . . . . . . . . 99 
3-46 Radon transform of waves traveling on the Empty shell using $0^{\circ}$ incidence data. Isolated compressional slownesses. . . . . . . . . . 100

3-47 Magnitude of spectra of first forward and backward compressional waves.101

3-48 Estimate of magnitude of compressional Reflection coefficient at stern endcap of Empty shell from $0^{\circ}$ incidence data. . . . . . . . . . 102

3-49 Radon transform of waves traveling on the Ribbed shell using $0^{\circ}$ incidence data. . . . . . . . . . . . . . . . . . 104

3-50 Radon transform of waves traveling on the Complex shell using $90^{\circ}$ incidence data. . . . . . . . . . . . . . 105

4-1 Forward modeling structure. . . . . . . . . . . . . . . 108

4-2 Example of a transmission line model for the Empty shell. . . . . . . 111

4-3 Incident wave approaching an impedance. . . . . . . . . 115

4-4 Reflected, transmitted and coupled waves leaving an impedance. . . . 115

4-5 Example 1 of transmission line model. . . . . . . . . . . 116

4-6 Example 2 of transmission line model. . . . . . . . . . . . . 117

4-7 Example 3 of transmission line model. . . . . . . . . . . . . . . 118

4-8 Example 4 of transmission line model. . . . . . . . . . . . . 119

5-1 Focused Empty shell data used for match to Transmission line. Center of shell only, time traces de-weighted by average energy. . . . . . . . 127

5-2 Transmission line model for first compressional wave on Empty shell. 128

5-3 Transmission line model of first compressional wave on Empty shell. . 129

5-4 Transmission line model for reflected compressional waves on Empty shell. . . . . . . . . . . . . . . . . . . . . . . . . . . . 129

5-5 Transmission line model of reflected compressional waves on the Empty shell. . . . . . . . . . . . . . . . . . . . . . 131

5-6 Transmission line model for early-time flexural-induced compressional waves on the Empty shell. . . . . . . . . . . . . . . . . . 133

5-7 Transmission line model of early time returns on Empty shell. . . . . 134 
5-8 Transmission line model for late-time flexural-induced compressional waves on the Empty shell. . . . . . . . . . . . . . . . 136

5-9 Transmission line model of early and late time returns on the Empty shell. . . . . . . . . . . . . . . . . . . . . . . 137

5-10 Focused Empty shell data used for match to Transmission line. Center of shell only. . . . . . . . . . . . . . . . . . . . . 138

5-11 Transmission line model for the Ribbed shell. . . . . . . . . . . . . . 139

5-12 Focused Ribbed shell data used for match to Transmission line. Center of shell only. . . . . . . . . . . . . . . . . . . . . . . . . . 140

5-13 Transmission line model of the Ribbed shell. . . . . . . . . . . . . . 141

5-14 response of shell surface, energy integrated over space. Ribbed shell for $0^{\circ}$ incidence case, conventional (bold) and transmission line model (plain) . . . . . . . . . . . . . . . . . . . . . . . 142

5-15 response of shell surface, energy integrated over time. Ribbed shell for $0^{\circ}$ incidence case, conventional (bold) and transmission line model (plain) . . . . . . . . . . . . . . . . . . . . . . . . . 14 


\section{Chapter 1}

\section{Introduction}

\subsection{Motivation}

Avoiding detection by adversaries has always been of great strategic military value. Accurate information about an enemy's position, strength and movement provides the key to the formulation of advantageous defensive and offensive maneuvers. For this reason, preventing the detection of one's own forces hampers an adversary's ability to make winning strategy. It also greatly enhances one's own by adding the element of surprise.

Technology has always played a vital role on both sides of the military long-range detection problem. As one side discovers a new detection technique, the other invents new countermeasures to defeat its advantage. In fact, since military technology among adversaries eventually equalizes through theft, copying, espionage, or purchase, the developer of such a technology also is forced to develop countermeasures to it in the eventuality that it will be used against him. For example, the engineers and scientists who developed Radar during WWII are the same people who later found that it was necessary to develop radar absorbent coatings and low cross section designs for their own aircraft in order to reduce their detectability by others with similar systems. This process continues in a never-ending upward spiral of technology, requiring detection and countermeasure systems of increasing complexity over time.

This same has been true in the world's oceans. It was quickly discovered that 
electro-magnetic radiation is completely absorbed by seawater within a few wavelengths. This renders all radar, IR and visible systems useless for the detection of underwater vehicles. During WWI and II, German U-boats used this stealth capability to wreak havoc on British merchant ship supply lines [1]. Only the development of sonar detection systems was able to bring the threat under control.

The fact that only fairly low frequency sound waves are capable of long range propagation and the presence of many of its own and numerous adversaries' submarines in the worlds oceans have made acoustics of major importance to the US Navy. The drive by the US Navy to make its own vessels quieter was heightened particularly by the cold war with the USSR, when the mission of the ballistic missile submarine became to remain hidden near its target until such time as it might be needed. The fundamental principle which guides submarine design and operational strategy is that the vulnerability of the submarine is directly proportional to its acoustic signature.

And so, there is a great deal of interest in the structural acoustic properties of ship and submarine hulls. To this end the Office of Naval Research is funding many efforts aimed at understanding the fundamental acoustic scattering processes of a submerged hull. Armed with such knowledge, designers can either avoid acoustically problematic structural arrangements or compensate for them.

For several reasons, this interest is currently focused in the mid-frequency range, $\frac{1}{2}<k a<10$, where $k=2 \pi / \lambda, \lambda$ is the acoustic wavelength in the water and $a$ is a characteristic length of the object. First, full scale test facilities exist and have provided empirical information about such structures in the low frequency, $k a<<1$, and high frequency, $k a>>1$, ranges. Second, there is a great deal of theoretical support in these regimes: Raleigh and Born approximations [2] at low frequency and the Geometric Theory of Diffraction [3, 4] at high frequency. Finally, numerical methods are computationally possible in these regimes. Thus, Finite Difference methods have tractable grid sizes at low frequencies and Ray tracing becomes valid at high frequencies. All of this leaves a large gap in the understanding of these structures in the mid-frequency regime.

The hulls of these vessels are essentially submerged, air-filled, finite, elastic cylin- 
drical shells. The shells are thin, with thickness to radius ratios of typically $1 \%$. They are constructed over bulkheads, rigid rib-like structures which support the thin hull membrane. They are further complicated by internal machinery and decks which are always resiliently mounted to the bulkheads. The internal structure to hull mass ratio is significant, on the order of 3 to 5 for typical submarine designs. Therefore, it is hypothesized that these discontinuities and mass loads will affect the acoustic properties of the vessel at mid-frequencies where wavelengths are on the order of the sizes of the the discontinuities.

In order to help the Navy understand the fundamental acoustic wave processes at work in a submarine hull at mid-frequency and to determine the effects of internal loading and hull discontinuities, the MIT Structural Acoustics Group has executed a number of mid-frequency, bistatic, scattering experiments on a set of model, finite, cylindrical shells. The simple shell models used in the experiments are not meant to mimic submarine design, but are designed in an incremental way to represent the fundamental acoustic components of the real structures.

The goal of this thesis is to extract information about these structural acoustic wave processes from the experimental data. The important parameters of these processes include wavespeeds, dispersion relations, decay rates and reflection, transmission and wave coupling coefficients at discontinuities. Measurements in the scattered acoustic field of these models contain much of this information. However, it is buried in the complex interaction occurring between the fluid and structure, including the propagation path to the measurement system. The sound field at the collection array therefore must be decomposed into fundamental, understandable pieces, via array processing, signal processing and forward modeling. This task is complicated by the mid-frequency nature of the data where both time and frequency resolution are poor.

\subsection{Previous Research}

The set of shells described in this paper was first investigated in backscatter from a previous set of monostatic experiments. The interesting incident angles where quasi- 
longitudinal and quasi-shear waves couple onto the shell through phase matching were identified by Corrado [5]. His preliminary studies provided basic knowledge which guided my further investigation of membrane waves on the shell. In addition, he identified helical shear waves as the major contribution to the backscattered field near beam incidence. At the writing of this thesis, another set of experiments is being conducted by MIT to investigate the damping of these waves by sandwiching a visco-elastic layer between two structural layers.

Mackovjac [6] processed the bistatic experimental data with Maximum Likelihood beamforming to identify structural resonances in the data. His processing is limited to the late-time portion of the data and a plane wave approximation. The resolutions he attains can be understood in the light of the Cramer-Rao bounds calculations done in Section 3.7 of this thesis.

Acoustic holography has been done on a much simpler set of Empty shells providing both pressure and velocity measurements on the surface of the shells $[7,8]$. Results from these experiments show the same types of features that are observed in our Empty shell.

The effect of internal loading on the scattering from infinite shells has been studied analytically by Achenbach [9] and Guo [10] and has been found to be significant. In particular, the location of attachment and the nature of the attachment, pinned versus rigid, has been shown to greatly affect the scattered field due to interaction with structural resonances of the shell.

Finally, Ricks [11], also of the MIT Structural Acoustics Group, has a numerical, full-elastic layered, cylinder model which is capable of computing the response of infinite cylinders to ring forces. Ricks is also investigating the effects of including a flat plate bulkhead and may be able to provide a benchmark for the results in this thesis in the near future [12]. 


\subsection{Hybrid Processing Approach}

There are many possible approaches which can be used to analyze a large experimental dataset such as the one described in this thesis. However, most processing tends to fall into one of two broad categories, either direct analysis or forward modeling. Each of these two approaches implies a specific processing structure and a set of advantages and disadvantages.

The structure of what can be called the direct analysis method is shown in Fig. 1-1. In this approach, the experimental data is considered to be the input to the processing chain. Direct analysis is then used to extract estimates of the physical variables of interest, which are the output of the processing chain. Examples of methods which fall into this category include beamforming, spectral analysis and linear filtering.

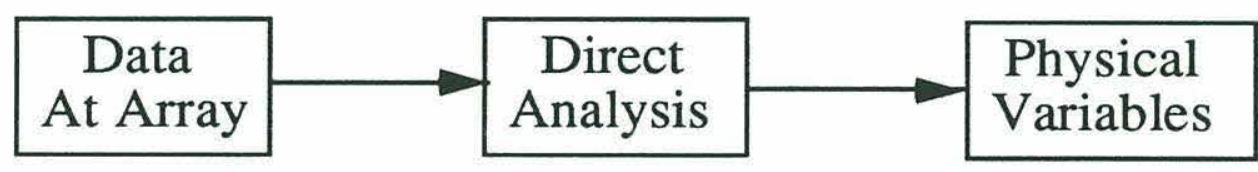

Figure 1-1: Direct analysis approach.

There are many advantages to this type of approach. The analysis is usually simple, analytically tractable and computationally efficient. It is also generally performed only once on a dataset, such as an FFT to determine spectral content. There are a large number of direct analysis methods to chose from, each complete with sizable body of literature.

However, there are several disadvantages to this type of approach. Generally, the accuracy of the physical variable estimates is limited by some parameter of the data collection, such as resolution of the measurement array, the sampling rate, data quantization or the signal to noise ratio (SNR). These methods, usually data transforms, are subject to processing artifacts. Aliasing, sidelobes and other process-introduced signals often contaminate results. And finally, even if these processing concerns are adequately addressed, the results given by such processing usually are not expressed in terms of the physical variables of interest. For example, a spectral analysis of the acoustic field received at a hydrophone array will quantify the energy present 
at a particular frequency, but cannot separate, say, the amplitudes of forward and backward traveling waves.

The structure of the forward modeling or matched-field approach is shown in Fig. 12. In this case, estimates of the physical variables of interest are used as the inputs to a model which outputs synthetic data. Then the estimates and the model are adjusted until the synthetic data matches the collected data. Finite Element, Boundary Element and Wavenumber Integral modeling all fall into this category.



Figure 1-2: Forward modeling approach

One advantage of this approach is that it is not limited by the parameters of the data collection. Since models are defined exactly, the model output can in theory be calculated to essentially "infinite" resolution and precision. Also, unlike direct analysis, by tracing the answers back through the model, it is usually possible to understand the fundamental parameters of interest. In fact, this technique is often used when both the input and the output are known. For example, in long-range ocean propagation problem, the input source characteristics are simple but the ray paths are of interest. If the input is not known, the method is even more valuable. Once a good match is made, the process provides both an estimate of the physical parameters and a model of the system being studied. Once this model is identified, the user can then explore it with new parameters to investigate similar problems. Use of this method is also valid when the actual system is too complex to model completely, the major contributions can be modeled and major features only matched. This allows partial understanding of complicated systems.

This structure too has its limitations. Unlike the direct analysis approach, this process is generally repeated many times either by hand or automatically using a feedback loop to adjust the parameters based on the data match. This can take many iterations or, in the worst case, if the model is not accurate enough, it may not converge at all. Also, although both direct analysis methods and models are 
generally available in computer libraries and usable in very generic forms, forward modeling usually requires a good deal of hand coding to match specific problems. High model complexity is often required to match even the coarsest features of real data. The complexity of the model is often several orders of magnitude greater than the direct analysis approach and these more complicated schemes have a tendency towards numerical instability and other unidentifiable artifacts. In addition, the model required is sometimes too complicated to compute. Current Finite and Boundary Element methods require more computing power than is currently available to compute even canonical problems, much simpler than the complicated geometries like those present in the experiments described in this thesis. Finally, it is often necessary to model many "uninteresting" processes in order to match the data, particularly if the "uninteresting" processes dominate. For example, it would be necessary for a Finite Element code to model the propagation path from the shell to the collection array, even though that path is well understood. This is not an interesting part of the problem, but it must be included in the model for the data to match at all. These kinds of issues greatly increase the model complexity.

Notice that the advantages and disadvantages of these two types of approaches are for the most part non-overlapping. This suggested a hybrid approach to me. Ideally, it would be best to take advantage of only the favorable characteristics of each of these types of methods and avoid the problematic characteristics completely. This is of course impossible in practice. However, it is possible to use one approach for that part of the processing where its strong characteristics produce the most benefit and switch to the other approach when the first approach begins to have problems. In this case, the approach should use low resolution direct analysis to eliminate the "uninteresting" features of the data and then use forward modeling to model only the interesting features at high resolution. A block diagram of the Hybrid Processing Structure which accomplishes this goal is shown in Fig. 1-3.

I have chosen the waves traveling on the shell as the center of the Hybrid Processing Structure. It is the output of both the direct analysis and forward modeling and is therefore common to both legs. I have chosen this space as the central output, 


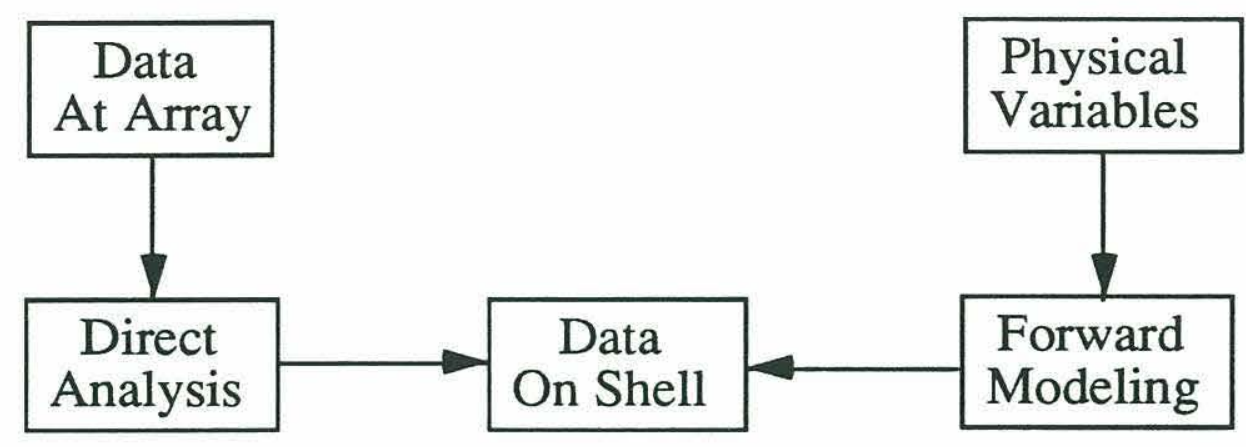

Figure 1-3: Hybrid approach

because it is an intuitive space in which to think about the fundamental physical processes at work on the shell. Waves traveling on the shell can be interpreted qualitatively after the direct analysis. Then the modeling can be used to complete those interpretations and quantify physical variables of interest.

There are several benefits to this processing structure in addition to the benefits listed above for each leg. The model used in the forward modeling leg can be simple and readily computable because the direct analysis eliminates the "uninteresting" features which complicate modeling. The low resolution of the direct analysis now does not limit the estimation of the physical variables of interest as it has been decoupled from the problem by the forward modeling. This hybrid structure removes the major obstacles posed by either approach alone.

This Hybrid Processing Structure is the major contribution of this thesis. Note that this is not a traditional analysis/synthesis approach. That type of approach is embodied in the forward modeling leg alone. The direct analysis leg has supplemented that traditional approach and increased its robustness for solution.

\subsection{Organization of Thesis}

This chapter has provided some motivation for this thesis, background information on the problem and an outline of my basic processing approach. The remainder of this document outlines the details of the approach and presents the results obtained by its application. 
Chapter 2 describes the various array processing methods used to implement the direct analysis leg of the Hybrid Processing Structure. This chapter begins with a description of the three shell designs, followed by a description of the experimental geometry, which will motivate the need for array processing. Section 2.2 continues with the description of the conventional beamformer which lays out the terminology and the theoretical framework for the non-adaptive, conventional focusing method in Section 2.3, the major processing tool of this direct processing step. An adaptive beamformer, the Maximum Likelihood Method, is also briefly formulated in Section 2.4 for later comparison and computation of resolution limitations. Additional direct processing, the Radon Transform, is described in Section 2.5. The Radon transform is a generalized 2-D Fourier transform used in the Seismic community. It is useful in this context for separating wavetypes.

Chapter 3 illustrates the results obtained with the various direct methods described in Chapter 2. The raw data from the shell is presented in Section 3.1 to indicate the problem at hand. The methods of Chapter 2 are applied to the data to yield the components of the dynamic structural response of the shell which are observable at the array. These methods are aided by source pulse deconvolution, array weighting and removal of the geometrically scattered return. These are described in Sections $3.2,3.3$ and 3.4. The resulting processed data clearly shows the radiating waves traveling on the shells. The complete processing chain is applied to all available data and presented in Section 3.5 along with a discussion of the major features of each dataset. The locations of discontinuities and their effects on the partitioning of energy within the shell bays is shown via the integration of energy along the time and spatial axes of the data in Section 3.6. The discontinuities in the shells give rise to reflections and transmissions that can be seen but not quantified due to the limits of the data time and spatial resolution. The resolution limit of the processing is quantified via comparison with the Maximum Likelihood beamformer and the Cramer-Rao bounds of the focusing process in Section 3.7. Comparison of this result with the integrated energy representation shows a discrepancy between actual and theoretical resolution which is later resolved via forward modeling. Finally, a crude 
estimation of the Reflection coefficient for compressional waves at the endcap in the Empty shell is made using the Radon Transform in Section 3.8. The poor quality of this estimate typifies the use of the direct approach alone to solve this type of problem and motivates the need for forward modeling.

Chapter 4 describes the models and parameter estimation methods used to implement the forward modeling leg of the Hybrid Processing Structure and circumvent the resolution limitations. The general structure of the parameter estimation process is discussed in Section 4.1. It mainly consists of a model and a parameter adjustment process. Section 4.2 describes the motivation for and the details of the Transmission Line model used to represent the shell. Several examples of the capabilities of this model are included. Finally, Section 4.3 describes the Simulated Annealing method used to adjust the parameters of the model to match the data.

The transmission line model described in Chapter 4 is used in Chapter 5 to match the focused data computed in Chapter 3 for the $0^{\circ}$ incidence case. This is accomplished through a step-by-step process which slowly increases the complexity of the model. Section 5.1 matches the parameters which pertain to the compressional waves traveling on the Empty shell. Sections 5.2 and 5.3 describes the models used to match the early and late time flexural-induced returns respectively. Finally, Section 5.4 shows the model used to match the data from the Ribbed shell at $0^{\circ}$ incidence. The integrated energy plots for this case clear up the discrepancy between observed and theoretical resolution brought up in Section 3.7.

Chapter 6 provides a summary of the major results of the thesis and recommendations for future work. 


\section{Chapter 2}

\section{Array Processing}

\subsection{Motivation}

Sound is intrinsically four dimensional. The signals that our ears receive vary as we move about space in three dimensions and as time passes. Both the time and spatial structure of a sound field carry a great deal of information. For example, if we were to record the electrical output from a single microphone in a room over time, we could tell from the spectrum of the signal if there were a periodic source present, such as a motor, a speaker, the gender of that speaker, the noise level etc. Spatial processing is equally powerful. This is evidenced by the fact that with only two array elements, our ears, sampling the sound field at only two points, we are able to localize a speaker in a room quite accurately. The same characteristics of sound that allow a person to focus on a speaker's location are the same characteristics that allow array processing to isolate sources of sound to different parts of the shell. This information is basically carried in the arrival time of a signal at an array element.

Array Processing is the spatial equivalent to Digital Signal Processing (DSP) in time as they both involve sampling a continuous process. DSP requires time sampling of signals and provides the mathematical formalism for frequency filtering operations. Array processing requires spatial sampling of the field and provides the mathematical formalism for spatial filtering operations. This formalism is identical for signal and array processing and in fact there would be no difference in their analysis if it were 
not for the physical limitations of arrays. The advent of computers has brought DSP to the forefront of signal analysis [13], since algorithms for processing digital signals only require software rather than an additional hardware investment as do analog algorithms. The hardware for time sampling of analog signals is fairly easy to acquire and once it is set up, an essentially infinite digital time series can be collected. The application of array processing mainly suffers from the constraints of the analog data acquisition part of the problem, since an additional physical sensor in general is required for each additional spatial sample. Because array elements are expensive and hard to place, arrays are often of limited lengths with few samples; often the sensors are not even equally spaced. This is in contrast to time sampling which has a large body of literature dedicated to processing of long, equally spaced digital time records. And finally, array processing is often complicated by the fact that arrays often sample over 2 and 3 spatial dimensions. In most cases, these differences between time and spatial sampling require that novel approaches to array processing be devised.

This chapter describes the array processing techniques used to implement the direct analysis leg of the Hybrid Processing Structure described in Section 1.3. The need for array processing is motivated by the data collected in the bistatic scattering experiments. Section 2.1.1 provides a description of the three model shells used in the experiments and Section 2.1.2 describes the experimental geometry used to measure the acoustic scattered field from these shells.

Section 2.2 describes the conventional plane-wave beamformer, the basic and most general array processing operation, and provides a notational basis for the remainder of the chapter. This general processing structure is then specified to conventional focusing in Section 2.3, which is the major tool of direct analysis used in this thesis. Both the conventional beamforming and focusing are non-adaptive approaches to array processing as neither takes into account any information about the structure of the field to reduce ambiguities. An adaptive approach known as the Maximum Likelihood Method (MLM) which uses the cross correlation of the field between array elements as a priori information to optimize array response, is derived briefly in Section 2.4. This processing is included to derive an analytic resolution limit for the 
direct processing in Chapter 3 using the Cramer-Rao bounds. Finally a description of the Radon Transform is presented in Section 2.5. This will be used to separate forward and backward traveling waves on the shell to estimate reflection coefficients in Chapter 3.

\subsubsection{Model Designs}

The MIT Structural Acoustic Group effort is concentrated on a number of experiments conducted on three, 90:1 scale submarine models designed by Conti and Dyer [14] and built by the Naval Research Laboratory (NRL) to high tolerance. These shells are the source of all the acoustic phenomenon described in this thesis.

The first model is a finite, thin, air-filled, cylindrical shell constructed from a nickel alloy, Ni-200. The ends of the shell are closed with a spherical endcap connected to the shell by a short conical section. A mechanical drawing of this shell is shown to scale in Fig. 2-1. The model has Thickness to Radius Ratio, $t / a=0.96 \%$, Aspect Ratio, $L / 2 a=7.75$ and Ring Frequency, $f_{\text {ring }}=14 \mathrm{kHz}$. This model is designated the Empty shell throughout this thesis.

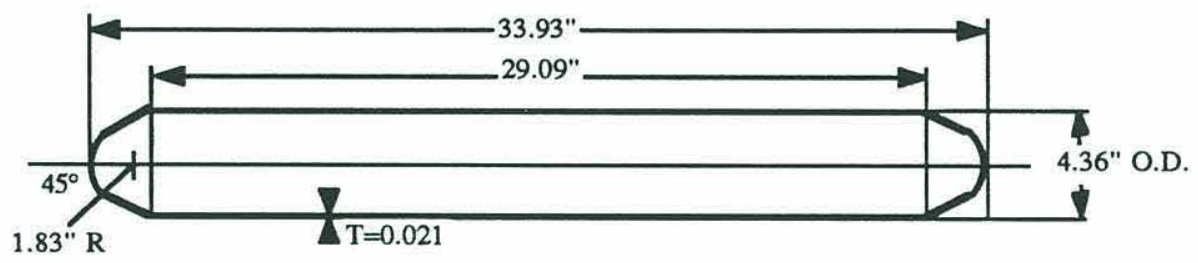

Figure 2-1: Empty shell design.

The second model is identical to the Empty shell except for the presence of four, massive, internal, nickel ring stiffeners welded to the shell. The total mass of the four rings is equal to the mass of the shell. These stiffeners are irregularly spaced and shown to scale in Fig. 2-2. This design was chosen over one with equally spaced ribs to eliminate ambiguities in the sound field due to structural periodicity. This model is designated the Ribbed shell throughout this thesis.

The third model is identical to the Ribbed shell but augmented by quadrant- 


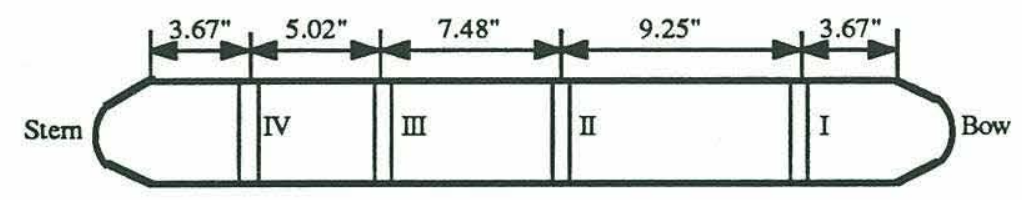

Figure 2-2: Ribbed shell design.

symmetric, resiliently-mounted, wave-bearing, internal structures. Four stainless steel masses are independently mounted to each ring stiffener with triangular blocks of EAR C1002 Isodamp rubber. An epoxy compound connects the rubber to the rings. A detailed drawing of the ring mounting is shown in Fig. 2-3. Corresponding masses

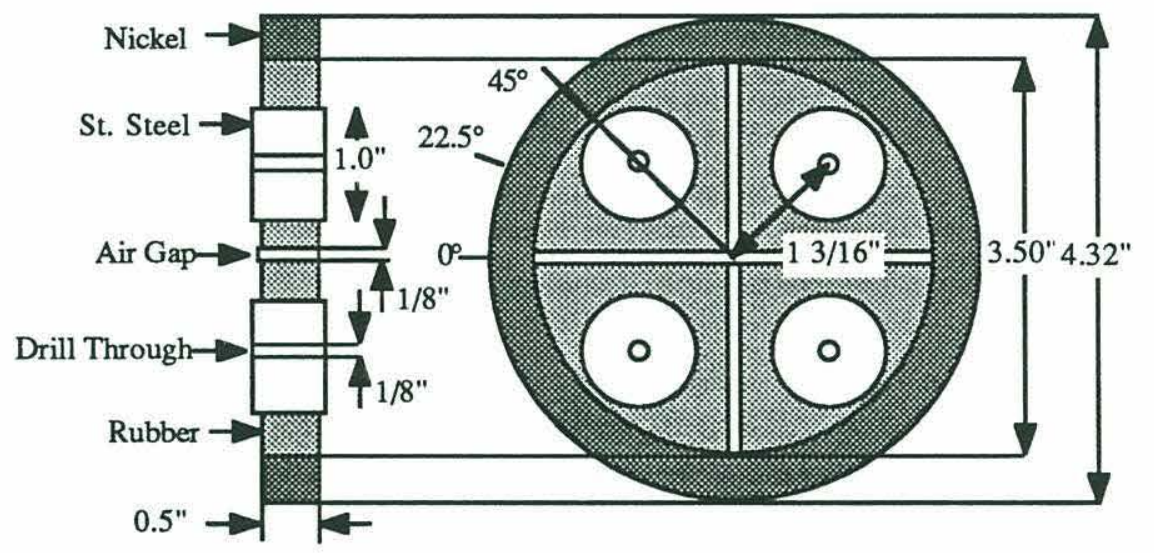

Figure 2-3: Ring and suspended mass system in the Complex shell.

in each ring are connected by Delrin rods, which have a measured quasi-longitudinal wave speed of $c_{l} \approx 1625 \mathrm{~m} / \mathrm{s}$. This arrangement allows waves to travel between the rings internally and is meant to simulate the low wavespeed paths in an actual submarine through machinery and decks. A typical pair of connected ribs is illustrated in Fig. 2-4. The ratio of the total mass of all rings and internals to the mass of the shell is approximately 3 , which is typical for submarine designs. The system of the rubber and the suspended masses were designed to have a natural frequency less than $1 / 3$ the ring frequency of the shell. This design is designated the Complex shell throughout this thesis. Further information on the shells, their construction and material properties can be obtained in Corrado [5]. 




Figure 2-4: Wave-bearing system in the Complex shell.

\subsubsection{Experiment}

The NRL Building 71 Scattering Measurement tank was used to acquire all data. The geometry of all experiments discussed in this thesis is shown in Fig. 2-5. The submerged shell was ensonified by a line array source $3 m$ in length at a distance of $2.18 \mathrm{~m}$ from the target center. The elements of the line array source were shaded to provide a plane wavefront incident at the shell. The source pulse was a wideband pulse with fairly uniform response from $10 k h z<f<50 k h z$. This pulse is described in greater detail in Section 3.2. The shell could be rotated in plane with respect to the source array to provide different angles of incidence. Bistatic data was collected for incident angles of $\theta_{i}=0^{\circ}, 66^{\circ}, 75^{\circ}$ and $90^{\circ}$ for reasons to be discussed in Section 3.1.1.

A single receiver was rotated $360^{\circ}$ azimuthally around the shell in plane with the shell center and source array at a distance of $2 \mathrm{~m}$ from the target center. Time records were recorded at 1 degree increments, providing a synthetic array measurement. The data was sampled at $500 \mathrm{khz}$, oversampled by a factor of approximately 5. 4096 time samples were collected after an approximately $2 \mathrm{msec}$ delay following the source firing. To reduce the contribution of incoherent signal components, 100 such time series were collected at each location and averaged. All measurements were calibrated and converted from receiver voltage levels into units of pressure $(\mathrm{Pa})$.

The measurement of the scattered field from the target was computed in the following manner. Data was acquired at all locations on the array as described above, 


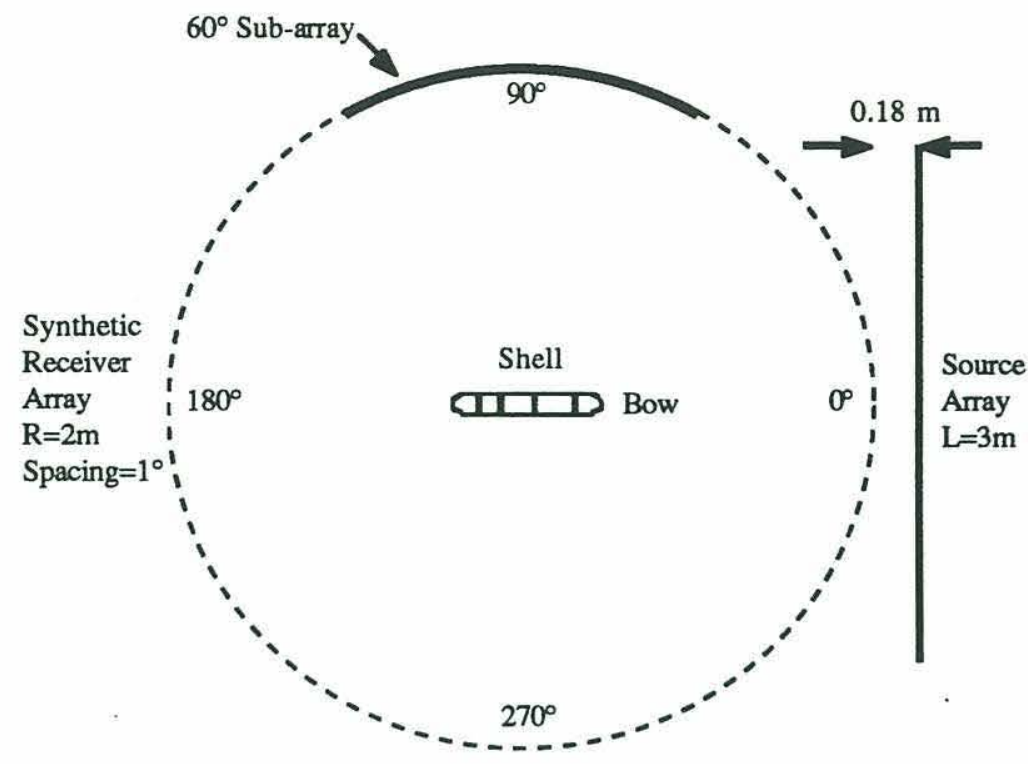

Figure 2-5: Experimental geometry.

first with the target in place and then with the target removed. This latter dataset provided a measurement of the tank clutter and the direct field from the source. It was subtracted from the former total field to form the scattered field measurement. Finally, a rectangular window with Hamming ends was applied to the time record to zero out target-dependent returns from the tank walls, floor and the water surface. Clutter subtraction is the limiting noise process, the SNR following this process is approximately $30 \mathrm{~dB}$. An ambient noise measurement shows that the background noise spectral level is $50 \mathrm{~dB}$ down from the signal level. Further details of data acquisition can be found in Corrado[5].

\subsection{Conventional Beamforming}

In order to understand the formulation of the conventional focusing algorithm and later the MLM algorithm, it is useful to develop some notation and the structure of the conventional beamformer. Since all the elements of the scattering experiment are in-plane with respect to one another, I will restrict my discussion to two dimensions and use a simple $x-y$ cartesian coordinate system with $x-y$ position vectors indicated in bold. 
In all experiments, the target, source and collection array are submerged in water, a homogeneous medium which supports no shear stresses. The pressure field, $P$, in this medium satisfies the homogeneous wave equation:

$$
\frac{d^{2}}{d t^{2}} P-c_{0}^{2} \nabla^{2} P=0
$$

where $\nabla^{2}$ in this case is the two-dimensional gradient operator and $c_{0}$ is the soundspeed in the water. Plane waves are one solution to this equation [2]. A single plane wave is defined as:

$$
\left.s(t, \mathbf{r})\right|_{\omega, k}=S(\omega, k) e^{j\left(\omega t-\mathbf{k}^{\top} \mathbf{r}\right)}
$$

where the pressure field, $s$, is a function of time, $t$, and a position vector, $\mathbf{r}$, at every angular frequency, $\omega$, and wavenumber, $k$. In a simple $x-y$ cartesian coordinate system, the wavenumber vector is derived from the wavenumber as, $\mathbf{k}=(|k| \cos \theta \hat{x},|k| \sin \theta \hat{y})$, where $\theta$ is the angle of arrival of the plane wave. In general, the spectral level, $S$ of the plane wave is dependent both on frequency and wavenumber.

The relationship between the angular frequency, $\omega$, and the wavenumber, $k$, is set by a propagation constraint from the governing wave equation for the medium. For homogeneous water, as in the experiment, the compressional soundspeed, $c_{0}$ is assumed to be constant over space and time. In this case, Eq. (2.2) is a solution to the wave equation if the wavenumber satisfies the dispersion relation:

$$
k=2 \pi / \lambda=\omega / c_{0}
$$

where $\lambda$ is the wavelength. Such a medium is known as non-dispersive. All frequencies travel at the same soundspeed so that energy packets traveling in the medium do not spread out over time. Later, this will not be the case for structural waves traveling on the shells.

The block diagram of the structure for a conventional beamformer is shown in Fig. 2-6. A field $s(t, \mathbf{r})$ impinges on a space populated with $N$ receiver elements at positions $\mathbf{z}_{n}$. Time series, $x_{n}(t)$, are collected at each receiver position and fed into 


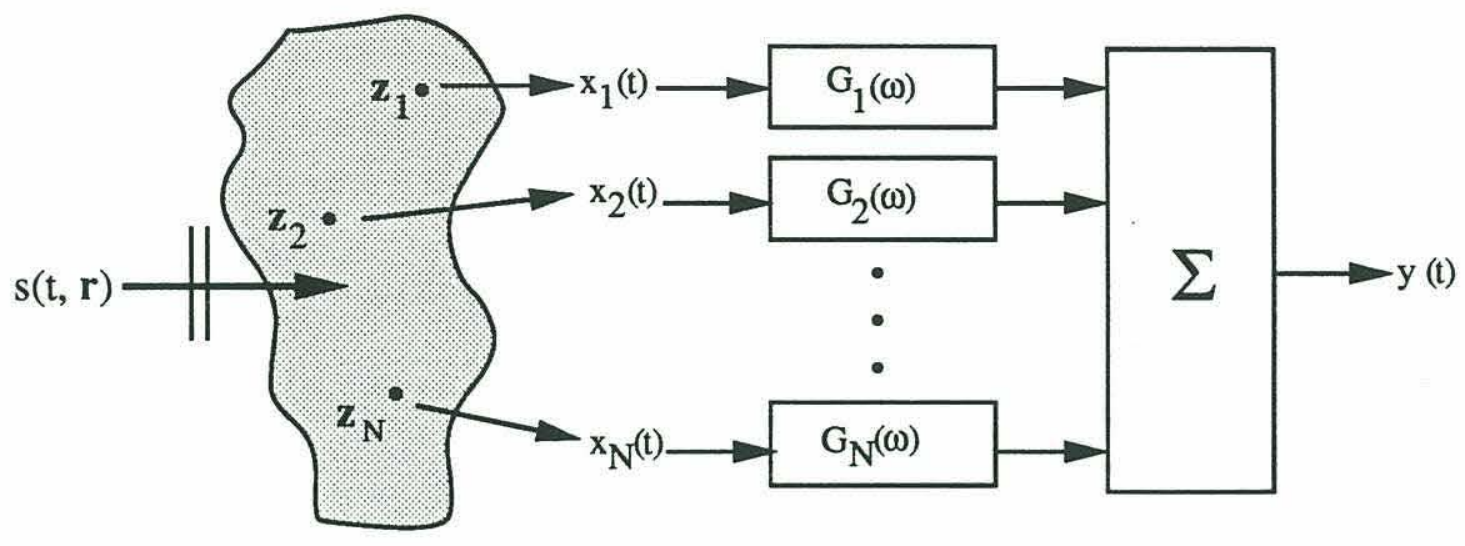

Figure 2-6: General beamforming structure

filters, $G_{n}(\omega)$, which generally consist of an amplitude weighting and a time delay. The outputs of these filters are added together to provide the output, $y(t)$. The output is determined by the characteristics of the filter components and the incoming field. Array processing which fits in to this structure is known as "delay and sum" beamforming.

Any signal, $s(t, \mathbf{r})$ can be broken down into a set of orthogonal basis functions in a plane-wave decomposition as follows:

$$
s(t, \mathbf{r})=\frac{1}{2 \pi^{D+1}} \int_{\omega} \int_{\mathbf{k}} S(\omega, k) e^{j\left(\omega t-\mathbf{k}^{\top} \mathbf{r}\right)} d \omega d \mathbf{k}
$$

where $D$ is the spatial dimensionality, in my case 2 . Therefore, $y(t)$ can be determined for each $\omega$ and $\mathbf{k}$, i.e. we can determine the response of the beamformer to each planewave, then the results are superposed for the final answer.

The output of the conventional plane-wave beamformer to a single unit-amplitude plane wave would be:

$$
\left.y(t)\right|_{\omega, \mathbf{k}}=\sum_{n=1}^{N} G_{n}(\omega) e^{j \omega t} e^{-j \mathbf{k}^{\top} \mathbf{z}_{n}}=\mathbf{E}^{\dagger}(\mathbf{k}) \mathbf{G}(\omega) e^{j \omega t}
$$


where:

$$
\mathbf{E}(\mathbf{k})=\left[\begin{array}{c}
e^{j \mathbf{k}^{\top} \mathbf{z}_{1}} \\
e^{j \mathbf{k}^{\top} \mathbf{z}_{2}} \\
\vdots \\
e^{j \mathbf{k}^{\top} \mathbf{z}_{N}}
\end{array}\right], \mathbf{G}(\omega)=\left[\begin{array}{c}
G_{1}(\omega) \\
G_{2}(\omega) \\
\vdots \\
G_{N}(\omega)
\end{array}\right]
$$

and $\dagger$ indicates conjugate transpose.

$\mathbf{E}^{\dagger}(\mathbf{k}) \mathbf{G}(\omega) e^{j \omega t}$ is known as the array response function, because it determines how the array will respond to incoming plane waves from various directions. $\mathbf{G}(\omega)$ is called the weight vector. This weighting is generally adjusted to decrease the sidelobes of the array response function. $\mathbf{E}(\mathbf{k})$ is known as the steering vector, since adjustment to the exponential modulation results in an adjustment of the major response axis of the array. The direction of maximal array response can be steered by offsetting the wavenumber in the steering vector. When the array is steered to wavenumber $\mathbf{k}_{\mathrm{t}}$, the response becomes:

$$
\left.y(t)\right|_{\omega, \mathbf{k}, \mathbf{k}_{\mathbf{t}}}=\mathbf{E}^{\dagger}\left(\mathbf{k}-\mathbf{k}_{\mathbf{t}}\right) \mathbf{G}(\omega) e^{j \omega t}
$$

In this case, if an incoming plane wave, $\left.s(t, \mathbf{r})\right|_{\omega, \mathbf{k}_{\mathbf{t}}}=S\left(\omega, \mathbf{k}_{\mathbf{t}}\right) e^{j\left(\omega t-\mathbf{k}_{\mathbf{t}}{ }^{\top} \mathbf{r}\right)}$, impinges on the receivers and the weighting vector is normalized such that, $\sum_{n=1}^{N} G_{n}(\omega)=1$, then the output $\left.y(t)\right|_{\omega}=S\left(\omega, k_{t}\right) e^{j \omega t}$, which is a unbiased estimate of the input. This phase delay is usually implemented by a delay in the filters, $G_{n}(\omega)$, but notice that it is mathematically incorporated into the steering vector, $\mathbf{E}(\mathbf{k})$ rather than in the weighting vector, $\mathbf{G}(\omega)$.

This formulation works for a plane-wave decomposition of the field. For any frequency, the output of this type of beamformer is generally a signal or signal power as a function of arrival angle. This angle is appropriate for truly plane waves. The plane wave assumption is valid in the far field of an object where the spherical wavefronts are of such large radius of curvature that they are essentially plane. However, the experimental data under consideration in this thesis were measured in the near field of the shells. Here the plane wave assumption is not a good one. The next section describes the conventional beamformer modified to eliminate this plane wave assumption. 


\subsection{Conventional Focusing}

The purpose of the direct analysis leg of the Hybrid Processing Structure described in Section 1.3 is to understand the waves traveling on the shell itself which, over time, re-radiate substantially through a large angular region. To do this, it is only necessary to remove the delay and superposition effects of the propagation path from the shell surface to the receivers. This will allow direct observation of the dynamic response of the surface of the shell which is capable of radiating into the far field.

Fortunately, the plane wave assumption is not appropriate at this particular measurement radius. If the measurements had actually been made in the far field, all the phase information in the field which allows spatial separation would have been lost. At beam aspect for this target, the near field range, defined by $L^{2} / \lambda$, is approximately $13 m$ at mid-band. The receivers, located at $2 m$ from the target center at beam, are therefore well within the nearfield of this shell as a whole. As a result, the field measured at the receivers contains phase information which allows the response of different parts of the shell to be separated.

At this point, a model for the field must be chosen. Many different models are possible. However, the idea is to choose a simple model which will be computationally tractable. Ideally, the best model would be one which includes all the traveling waves on the shell, radiation of these waves and propagation to the collection array. This would require matching the data generated by the forward model to data collected at the array which not only defeats the purpose of the Hybrid Processing Structure but would most likely be computationally impossible. Therefore, a simple model is

chosen here and later its resolution will be computed using the Cramer-Rao bounds of an optimal processor, the MLM.

Backpropagation requires coherent addition of the receiver elements for each position on the shell, also known as conventional focusing. The field is assumed to be generated by a finite number of virtual, point sources at points $\mathbf{z}_{i}^{\prime}$ on the shell with 
time response $s_{i}(t)$. The data seen at the $n$th receiver, located at $z_{n}$ is then given by:

$$
x_{n}(t)=\sum_{i} \frac{1}{4 \pi d_{n, i}} s_{i}\left(t-\frac{d_{n, i}}{c_{0}}\right)
$$

where $d_{n, i}=\left|\mathbf{z}_{i}^{\prime}-\mathbf{z}_{n}\right|$ and $c_{0}$ is the soundspeed in the water.

Each source signal can be reconstructed by combining the response from each of the receiver elements as depicted in Fig. 2-7. In the time domain, this conventional

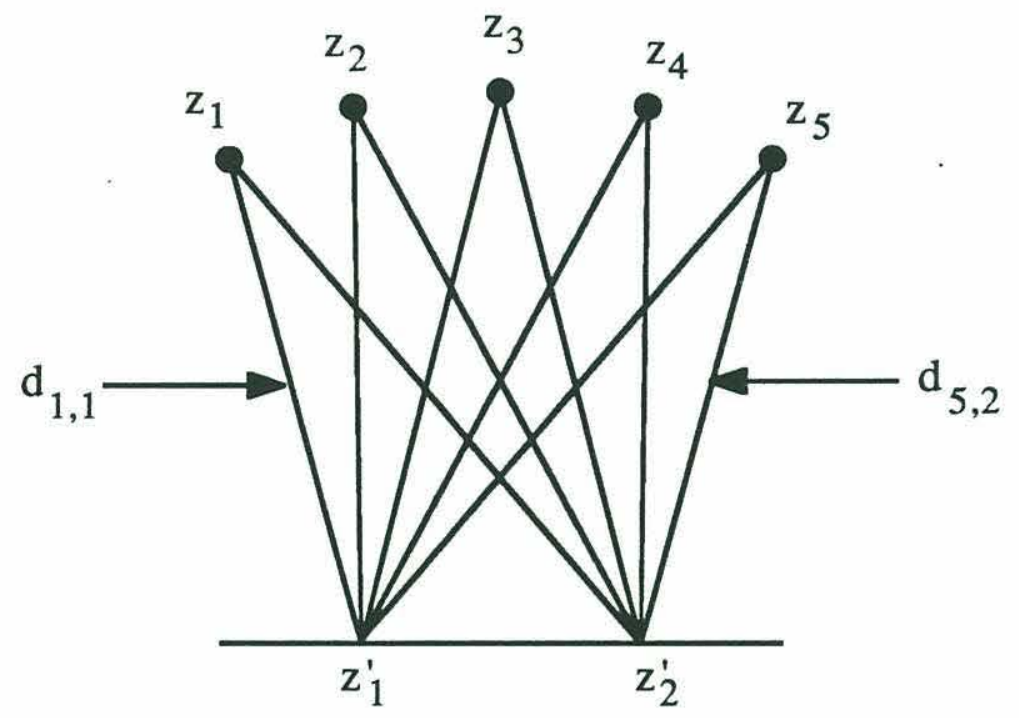

Figure 2-7: Intuitive focusing operation

focusing operation is defined:

$$
y_{i}(t)=\sum_{n=1}^{N} 4 \pi d_{n, i} x_{n}\left(t+\frac{d_{n, i}}{c_{0}}\right) G_{n}(\omega)
$$

where, as before, $G_{n}(\omega)$ is a weighting coefficient on each receiver to be used later in sidelobe reduction. $y_{i}(t)$ provides an estimate of the source response, $s_{i}(t)$. To see this, substitute the expression for $x_{n}(t)$ into Eq. 2.9:

$$
y_{j}(t)=\sum_{n=1}^{N} \sum_{i} \frac{d_{n, j}}{d_{n, i}} s_{i}\left(t-\frac{d_{n, i}-d_{n, j}}{c_{0}}\right) G_{n}(\omega)
$$

If $\sum_{n=1}^{N} G_{n}(\omega)=1$ and $s_{i}(t)=0$ for $i \neq j$, then $y_{i}(t)=s_{i}(t)$. Since more than one source generally radiates at the same time, the estimate at each point will be 
contaminated by the response from other points. The degree of contamination is determined by the resolution and sidelobe structure of the collection array.

A synthetic example of the results of this kind of focusing is shown in Fig. 2-8. Here the impulses from three sources overlap across the array. Focusing will constructively add the channels to synthesize the response from each source. This is an idealized example, in reality there would be sidelobes from the processing.

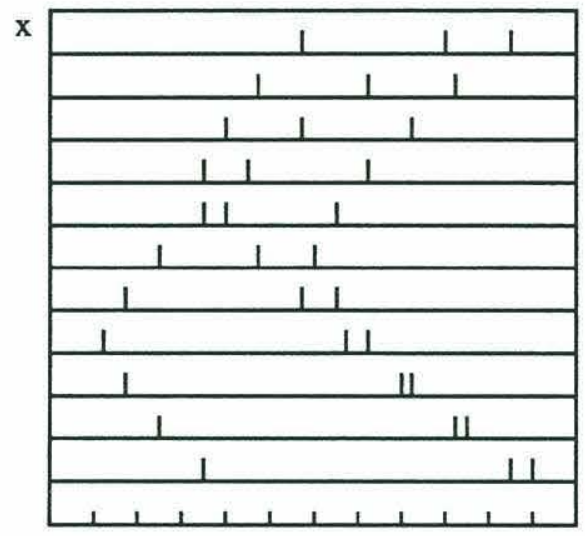

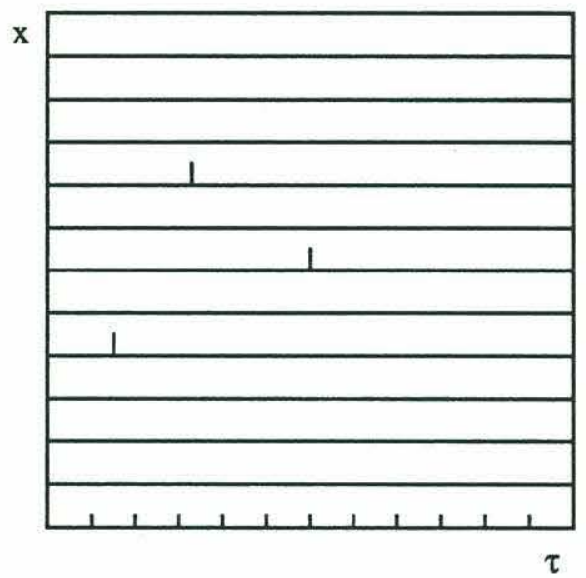

$\tau$

Figure 2-8: Synthetic focusing example

The formulation of conventional focusing can also be expressed in terms similar to the conventional beamformer. To see this, consider the response of a system which is focused at source $j$ measuring the field from source $i$ :

$$
\left.y(t)\right|_{j, i}=\sum_{n=1}^{N} \frac{d_{n, j}}{d_{n, i}} s_{i}\left(t-\frac{d_{n, i}-d_{n, j}}{c_{0}}\right) G_{n}(\omega)
$$

or in the frequency domain:

$$
\left.y(t)\right|_{\omega, j, i}=\sum_{n=1}^{N} \frac{d_{n, j}}{d_{n, i}} s_{i}(\omega) e^{j \omega t} e^{-j k\left(d_{n, i}-d_{n, j}\right)} G_{n}(\omega)=\mathbf{E}^{\dagger}\left(d_{n, j}-d_{n, i}\right) \mathbf{G}(\omega) e^{j \omega t}
$$

where:

$$
\mathbf{E}\left(d_{n, j}-d_{n, i}\right)=\left[\begin{array}{c}
\frac{d_{1, j}}{d_{1, i}} e^{j k\left(d_{1, j}-d_{1, i}\right)} \\
\frac{d_{2, j}}{d_{2, i}} e^{j k\left(d_{2, j}-d_{2, i}\right)} \\
\vdots \\
\frac{d_{N, j}}{d_{N, i}} e^{j k\left(d_{N, j}-d_{N, i}\right)}
\end{array}\right], \mathbf{G}(\omega)=\left[\begin{array}{c}
G_{1}(\omega) \\
G_{2}(\omega) \\
\vdots \\
G_{N}(\omega)
\end{array}\right]
$$


This mathematical structure carries over to the MLM formulation in the next section.

\subsection{MLM Focusing}

The beamformers described in the last two sections are non-adaptive in nature. By this, I mean that the weight vector, $\mathbf{G}(\omega)$ is fixed. However, this type of filtering is sub-optimal in a probabilistic sense, as it does not use the available a priori knowledge about the incoming signal to reduce sidelobes and improve the output response. A class of beamformers which does utilize this information are called adaptive. These filters generally require an the incoming signal covariance across the array.

One such adaptive beamformer is the Maximum-Likelihood Method (MLM). This "high resolution" beamformer has been studied in detail $[15,16,17,18]$ mainly because of its analytical tractability and because it can be shown that if there is an "optimal" solution to a problem, one which uses all a priori information, that solution is the Maximum Likelihood solution [19]. This beamformer will be used in Chapter 3 to show estimate the spatial resolution of the direct analysis. The concept behind this processor is quite simple. The first characteristic that is desirable for any beamformer is that the output is normalized to maintain energy in the target direction. In the matrix notation of the last two sections, this is expressed:

$$
\mathbf{E}^{\dagger}\left(\mathbf{k}_{t}\right) \mathbf{G}\left(\omega \mid \mathbf{k}_{t}\right)=1
$$

With this constraint in force, the output energy of the beamformer must be minimized:

$$
\min _{\mathbf{G}(\omega)} \mathbf{S}_{y}(\omega)
$$

where $\mathbf{S}_{y}(\omega)=\mathbf{G}^{\dagger}\left(\omega \mid \mathbf{k}_{t}\right) \mathbf{K}_{x}(\omega) \mathbf{G}\left(\omega \mid \mathbf{k}_{t}\right)$ and $\mathbf{K}_{x}(\omega)$ is the actual input covariance matrix across the array.

This is solved as a Lagrange multiplier problem using routine calculus of variations. 
The weight vector which satisfies these requirements is simply:

$$
\mathbf{G}\left(\omega \mid \mathbf{k}_{t}\right)=\frac{\mathbf{K}_{x}^{-1}(\omega) \mathbf{E}\left(\mathbf{k}_{t}\right)}{\mathbf{E}^{\dagger}\left(\mathbf{k}_{t}\right) \mathbf{K}_{x}^{-1}(\omega) \mathbf{E}\left(\mathbf{k}_{t}\right)}
$$

The MLM beamformer uses the information in the field to adjust the weight vector such that signals from any direction other than the a priori signal direction are rejected. This improves the sidelobe response of the beamformer and greatly sharpens the mainlobe as well.

For stationary, homogeneous processes the covariance matrix is given by:

$$
\mathbf{K}_{x}(\omega)=\mathbf{X}(\omega) \mathbf{X}^{\dagger}(\omega)
$$

where $\mathbf{X}(\omega)=\left(X_{1}(\omega), X_{2}(\omega), \ldots, X_{N}(\omega)\right)^{\top}$, the Fourier coefficient of the time series at each receiver. However in practice, the input correlation matrix must be computed with extreme care. The assumption made in this derivation is that the input signal is stationary and homogeneous. The wave components propagating on the shell generate arrivals at the receiving array which are transient and inhomogeneous. Therefore, it is necessary for this analysis to be performed over short time intervals. Time windowing of the input signals is used to accomplish this.

Since the time response of each array element must be windowed, it is necessary to compensate for the delays to the sensors. Otherwise, the windowing operation in the covariance matrix computation will exclude the actual time of interest. In a plane-wave formulation, the delays are simple stacking operations, see [20,21] for details. In the case of focusing however, the stacking operation must be replaced with a focusing operation. Notice that the same model is being used in this beamformer as in the conventional focusing operation. This will be important in the resolution computation.

The actual implementation of this method is as follows: For each virtual source 
location, $i$, on the shell the propagation delay and spreading loss are removed:

$$
x_{n}(t, i)=4 \pi d_{n, i} x_{n}\left(t+\frac{d_{n, i}}{c_{0}}\right)
$$

Short-time Fourier transforms are then performed:

$$
X_{n}(\omega, T, i)=\int_{T}^{T+T_{w}} w(t-T) x_{n}(t, i) e^{-j \omega t} d t
$$

Where $T_{w}$ is the window size and $w(t)$ is a time window used to control sidelobes. The vector $\mathbf{X}(\omega, T, i)=\left(X_{1}(\omega, T, i), X_{2}(\omega, T, i), \ldots, X_{N}(\omega, T, i)\right)^{\top}$ is formed. Then the input covariance matrix for each source, $i$, time, $T$, and angular frequency, $\omega$ is simply:

$$
\mathbf{K}_{x}(\omega, T, i)=\mathbf{X}(\omega, T, i) \mathbf{X}^{\dagger}(\omega, T, i)
$$

In general this matrix is close to singular. In order to calculate $\mathbf{K}_{x}^{-1}$ it is necessary to stabilize this matrix by adding a small amount of uncorrelated noise by modifying the diagonal elements.

$$
\mathbf{K}_{x}^{\prime}(\omega, T, i)=\mathbf{K}_{x}(\omega, T, i)+\epsilon \mathbf{I}
$$

where $\mathbf{I}$ is the identity matrix and $\epsilon$ is typically 1 percent of the geometric mean of the diagonal elements:

$$
\epsilon=0.01 \times\left(\prod_{i=1}^{N}\left(\mathbf{K}_{x}\right)_{i i}\right)^{\frac{1}{N}}
$$

\subsection{Radon Transform}

The Radon Transform will be used in Chapter 3 to estimate the reflection coefficient for compressional waves at the endcap of the Empty shell and prove the existence of axially traveling compressional and shear waves at $90^{\circ}$ incidence in the Complex shell. It was first mathematically formalized by Radon in 1917 and has been studied in detail since then $[22,23,24,25]$ in the context of seismic multi-layer reflection. It can either be described as a specialization of the Hough Transform [26], which transforms lines to points, or as a generalized Fourier transform, where the integration is done 
across lines of constant slope in the $k-\omega$ plane. The transform is invertible and is written as a sum over spatial locations delayed by position times a scanning slowness.

$$
\hat{F}(\tau, p)=\sum_{x=-\infty}^{\infty} f(x, \tau+p x)
$$

The Radon transform is also known as a plane-wave decomposition, since it is essentially a bank of conventional beamformers, each set to a different angle of arrival. If the array were horizontal, the Radon Transform would break down the arrivals over horizontal wavenumber, $k_{x}=k \sin (\theta)$, where $\theta$ is the angle of arrival. The transform is actually parameterized by horizontal slowness, $p_{x}=1 / c_{x}$. Since $k_{x}=\omega / c_{x}=\omega p_{x}$ the two are equivalent. An intuitive picture of this operation is shown in Fig. 2-9.
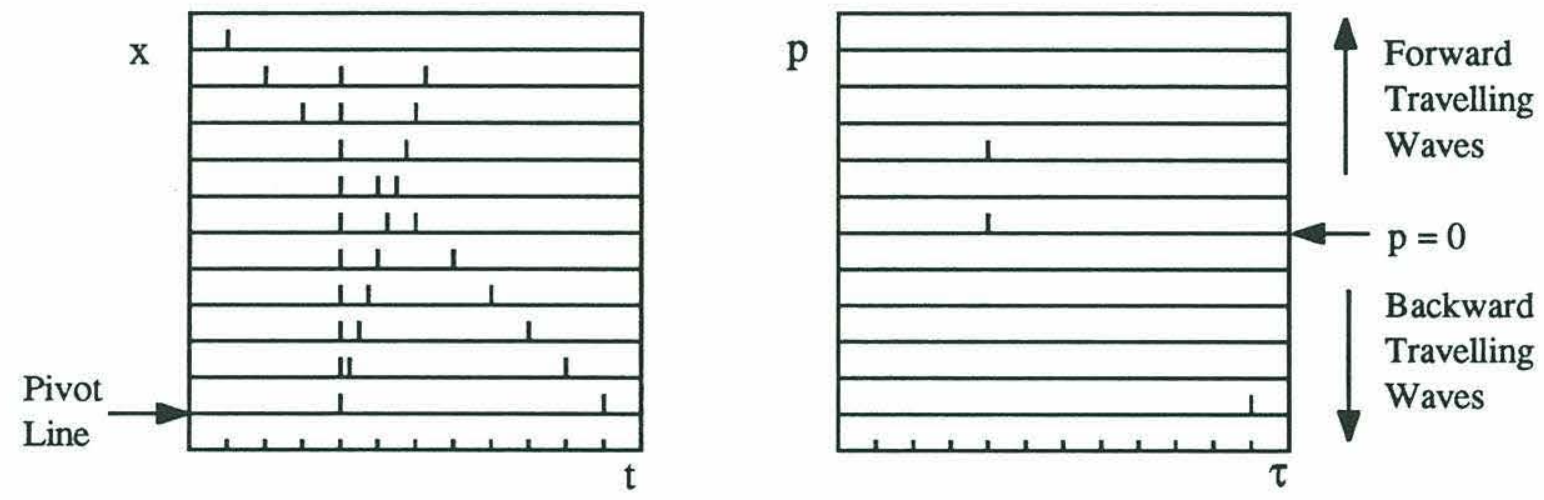

Figure 2-9: Radon Transform example

In this example, there is a signal which arrives at all receivers at the same time, this line in $x-t$ space is transformed to a point in $\tau-p$ space at zero slowness. The signal that has small positive moveout in $x$ for positive moveout in $t$ transforms to a small positive slowness. The signal with large negative moveout in $x$ for positive moveout in $t$ transforms to a large negative slowness. Implicit in this transform is a "pivot" line, which is the trace that is not shifted in time over the coherent addition. The parameter $\tau$ is known as the "intercept time." This can be thought of as the time the signal arrives at or "intercepts" the pivot line. 


\section{Chapter 3}

\section{Array Processing Results}

This chapter is devoted to the results obtained with the array processing methods described in Chapter 2. It describes the lengthy processing chain required to transform the collected data to a more intuitive information space: the waves on the shell surface. In the interest of unfolding the complexity of the data in a logical manner, the process that I used to arrive at the clean focused data on the shell will be described step by step and justified. This will illuminate both the assumptions made and the pitfalls of such data processing.

Section 3.1 presents the entirety of the raw data collected in this series of bistatic experiments and briefly describes the identifiable features they contain. The processing chain is developed by following the Empty and Ribbed shell datasets for the case of $0^{\circ}$ incidence at each stage of the processing. Section 3.2 shows the results obtained by removing the source pulse from the data via deconvolution. This operation increases the time resolution of the collected data. Conventional Focusing is used in Section 3.3 first with a rectangular and then with a Hamming tapered array weighting to transform the data into the space where the waves can be seen traveling on the shells. Unfortunately, these waves are obscured by the geometrically scattered return. The removal of this return to allow observation of the waves of interest is the subject of Section 3.4, which completes the processing chain. Section 3.5 shows the results of the full processing chain for all available data. Comparisons are made among the different shells and incident angles in this section. Section 3.6 describes 
energy measurements made on the focused data. These provide some insight into the resolution of the processing and some important shell parameters such as decay rates. Section 3.7 uses the MLM beamformer and the Cramer-Rao bounds to attempt to explain the spatial resolution of the processing. Finally, Section 3.8 uses the Radon Transform to estimate the reflection coefficient of compressional waves at the endcap of the Empty shell. The attempt to perform a similar calculation for the Ribbed Shell shows the limitations of direct analysis.

\subsection{Raw Experimental Data}

\subsubsection{Full Dataset}

The data collected at NRL is clutter subtracted and windowed of target-dependent tank wall, surface and floor returns as described in Section 2.1.2. This provides a clean measurement of the scattered pressure field from each shell at various angles of incidence.

The number of incident angles collected is limited for several reasons. The time available to MIT at the NRL tank was limited to about four calendar weeks total. The collection of a full $360^{\circ}$ bistatic dataset is time consuming, about 24 hours of active collection. Both the changing of incident angle and the changing of shells are done manually, requiring that staff be present. This for the most part limited the collection time to NRL business hours. Changing shells also required a $24 \mathrm{hr}$ target wetting period to allow degassing of the shell. Thus, a limited number of incident angle/shell combinations were chosen to fit the time allotted.

$0^{\circ}$ and $90^{\circ}$ were considered good limiting cases. $66^{\circ}$ was chosen because some previous monostatic experiments indicated it would be a good angle to excite shear waves on the shell [5]. These same experiments also indicated that both compressional and shear waves would be excited at $75^{\circ}$. Data was collected for the Empty shell for angles of $0^{\circ}, 5^{\circ}$ and $75^{\circ}$. Data was collected for the Ribbed shell for angles of $0^{\circ}$ and $75^{\circ}$. Data was collected for the Complex shell for angles of $0^{\circ}, 5^{\circ}, 66^{\circ}, 75^{\circ}$ and $90^{\circ}$. 
The set is incomplete due to the time constraint and occasional acquisition equipment anomalies.

It is useful to look at the raw time series to orient oneself to the task at hand and understand some obvious features. The raw time traces of the scattered field of the shell at all $360^{\circ}$ of the collection array, due to an incident plane wave from $90^{\circ}$ from the bow (beam aspect) are shown in Fig. 3-1. The horizontal axis is time in milliseconds from the time that the line source was fired. Time traces were collected every $1^{\circ}$, but this plot shows the time trace from every 6 th receiver for plotting resolution reasons. The vertical axis is an approximate angular receiver location. There are two major features here. At $180^{\circ}$ degrees there is a forward scattered return and at $0^{\circ} / 360^{\circ}$ there is a back scattered return. These returns fairly dominate all other processes on the shell. They are in a sense the least interesting as they are determined by the geometrically scattering component, which is well understood and can be actively damped in practice.

To look at the other regions of the scatter more closely, in Fig. 3-2 I have scaled each line of Fig. 3-1 individually from its minimum to its maximum value. This shows a characteristic " $w$ " pattern which is caused by the arrival time of the geometrically scattered return varying as the distance from the shell to the receiver changes, from $195 \mathrm{~cm}$ at beam to $115 \mathrm{~cm}$ at bow or stern. The location of this pattern shifts as the incident angle changes. Figs. 3-3, 3-4 and 3-5 show the the scaled raw data for the incident angle of $75^{\circ}$ for the Empty, Ribbed and Complex shells respectively. The Ribbed shell plot for this incident angle shows only receiver angles of $-105^{\circ}$ to $75^{\circ}$ by $3^{\circ}$ as only this range was collected for this dataset. For this angle of incidence, the forward scattered return has moved to $165^{\circ}$. Notice that features after the first, geometrically scattered, return change between different shells at the same angle as well as for the same shell at different angles.

Fig. 3-6 shows the raw data for the Complex shell at $66^{\circ}$. The forward scattered return moves to $156^{\circ}$ for this incident angle. Notice that the returns after the geometrically scattered return have less amplitude than those in the $75^{\circ}$ case. At this angle only shear waves can couple onto the shell, so there is less energy to re-radiate. 


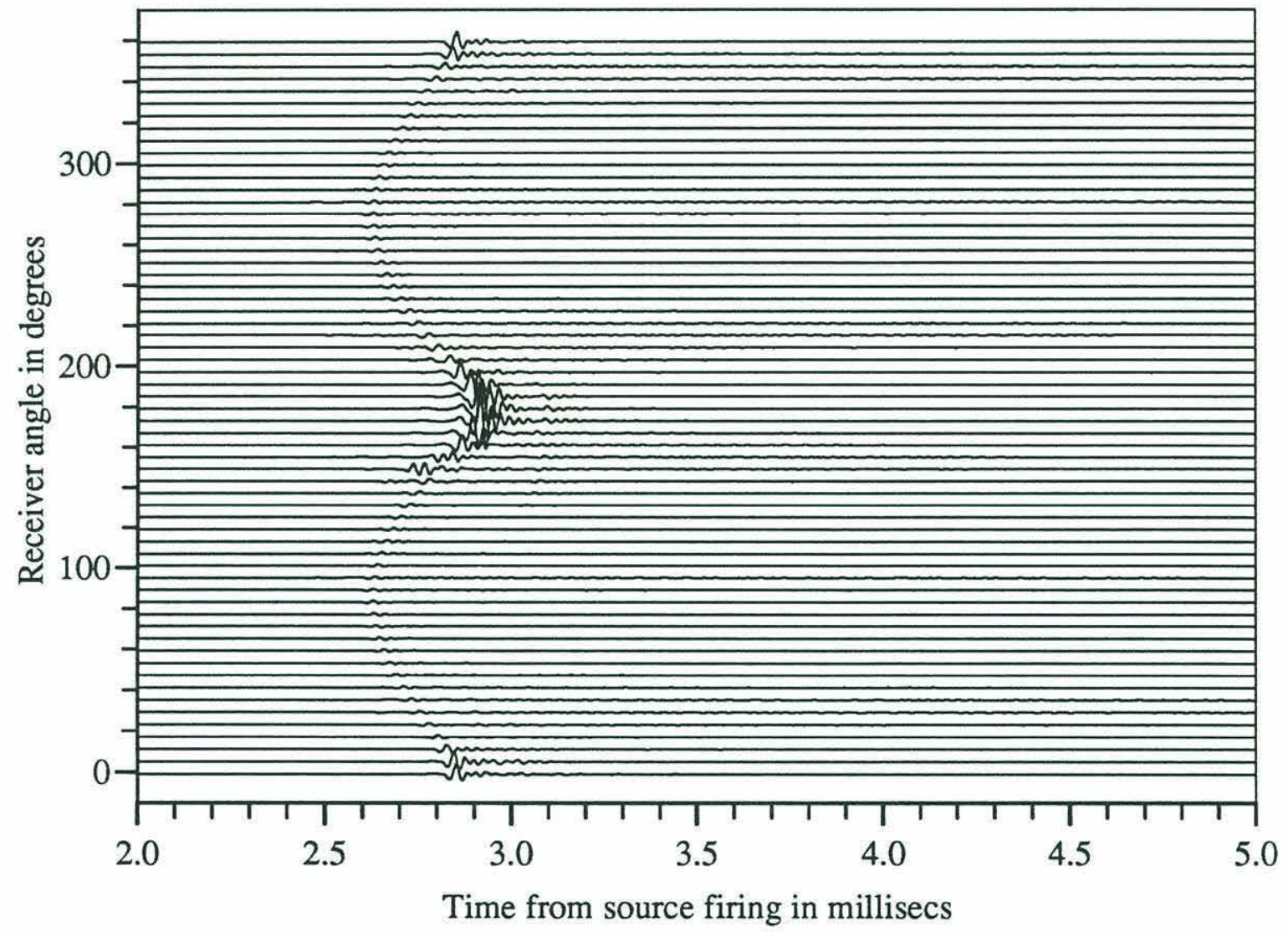

Figure 3-1: Raw data from the Complex shell. Incident angle $=90^{\circ}$. Receivers $0^{\circ}-360^{\circ}$ by $6^{\circ}$. 


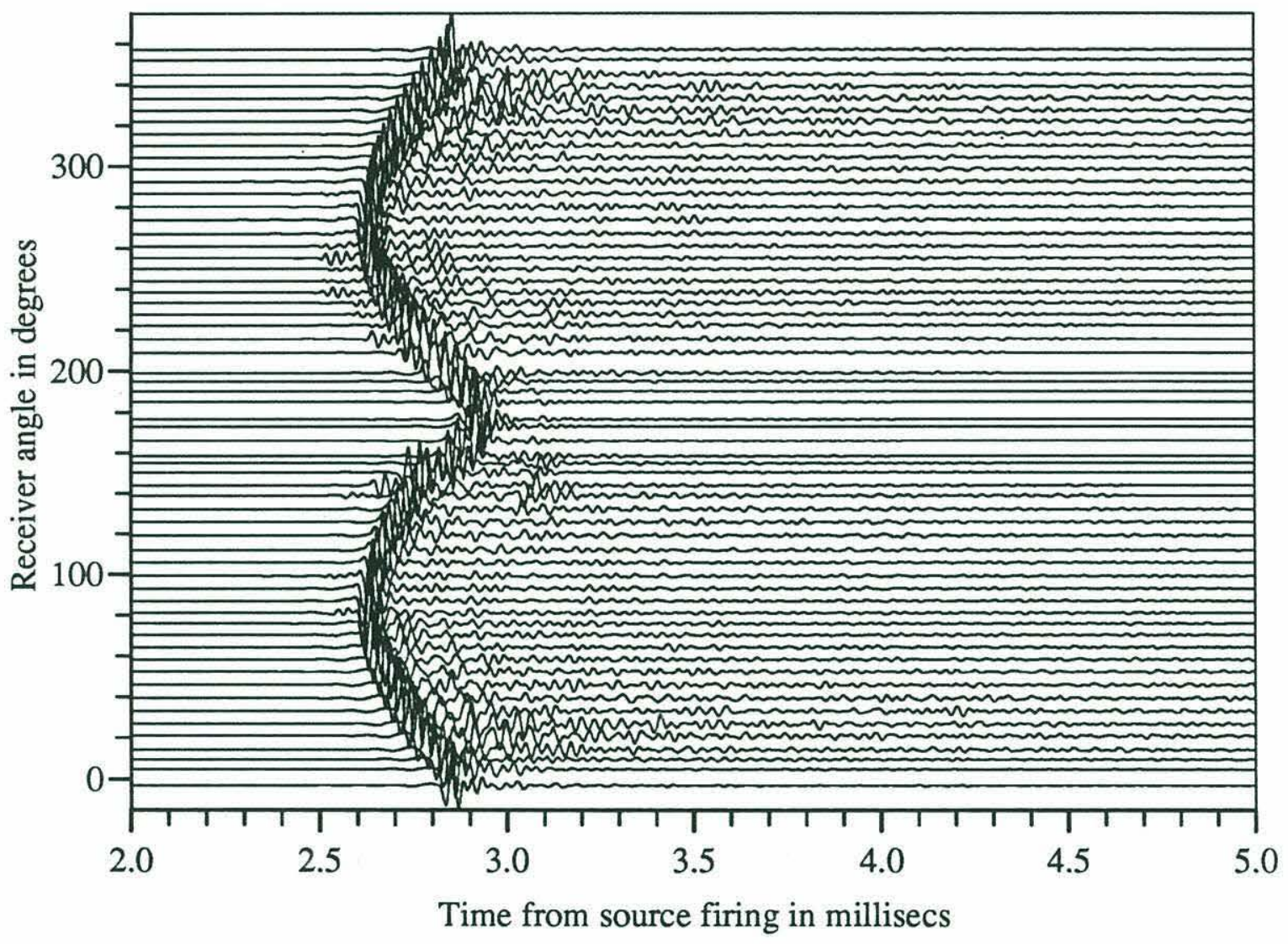

Figure 3-2: Raw data from the Complex shell. Incident angle $=90^{\circ}$. Receivers $0^{\circ}-360^{\circ}$ by $6^{\circ}$. Each line scaled min to max. 




Figure 3-3: Raw data from the Empty shell. Incident angle $=75^{\circ}$. Receivers $0^{\circ}-360^{\circ}$ by $6^{\circ}$. Each line scaled min to max. 


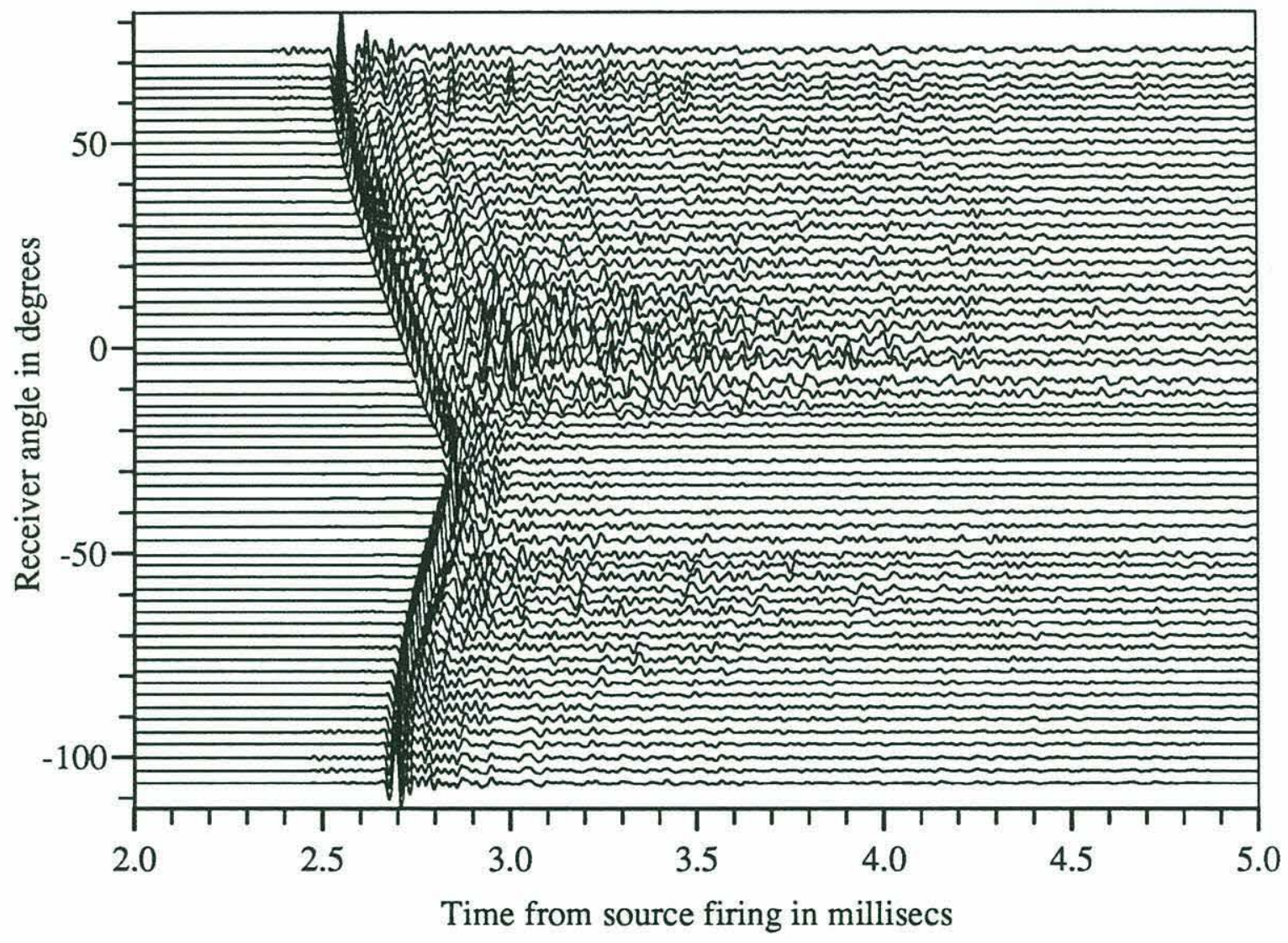

Figure 3-4: Raw data from the Ribbed shell. Incident angle $=75^{\circ}$. Receivers $-105^{\circ}$ $75^{\circ}$ by $3^{\circ}$. Each line scaled min to max. 


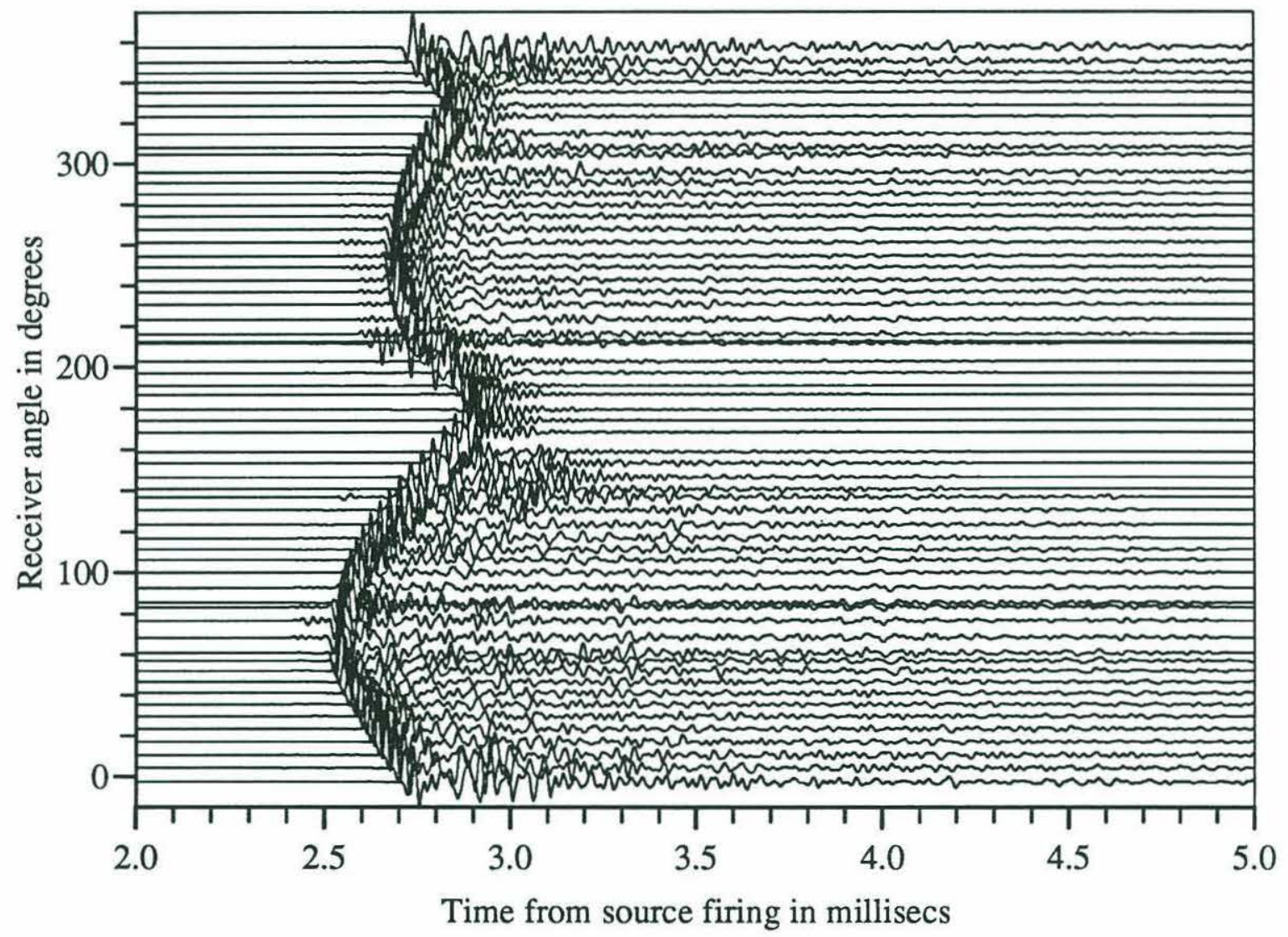

Figure 3-5: Raw data from the Complex shell. Incident angle $=75^{\circ}$. Receivers $0^{\circ}-360^{\circ}$ by $6^{\circ}$. Each line scaled min to max. 


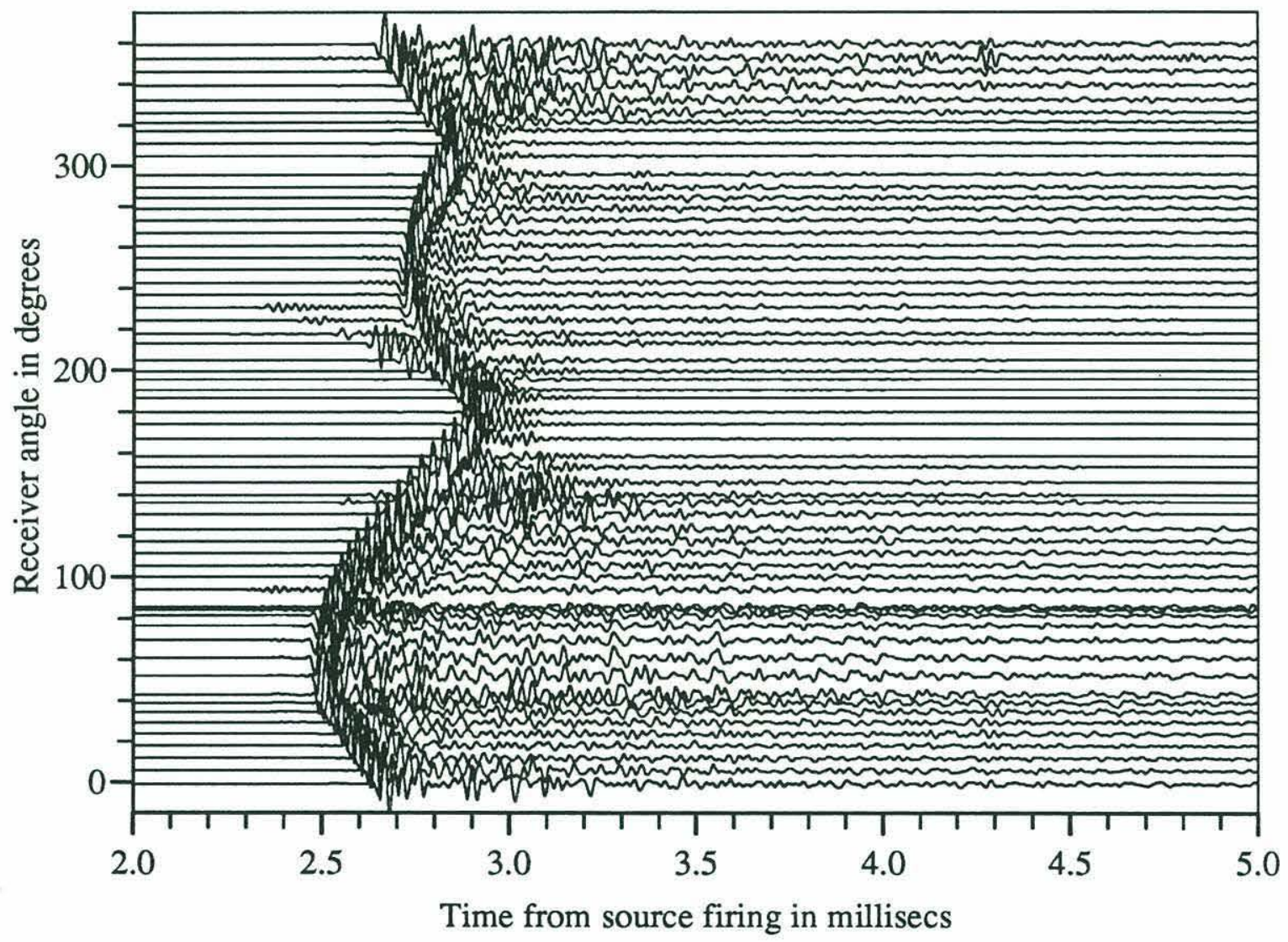

Figure 3-6: Raw data from the Complex shell. Incident angle $=66^{\circ}$. Receivers $0^{\circ}-360^{\circ}$ by $6^{\circ}$. Each line scaled min to max. 
To investigate axial incidence, the $0^{\circ}$ case was measured. At incident angles of $0^{\circ}$ and $180^{\circ}$, the shell is symmetric with respect to the source. Although the data was collected for a full $360^{\circ}$ around the target, the symmetry of the shell and the high tolerance of the measurement system gave close to symmetric responses between the port and starboard receiver array arcs. Therefore it is sufficient to look at a $180^{\circ}$ subarray. Figs. 3-7, 3-8 and 3-9 show the scaled raw scattered return from the Empty, Ribbed and Complex shells respectively for a $0^{\circ}$ incident pulse. Note that the forward scatter has moved to $180^{\circ}$. Once again there are differences between shells, with the greatest differences between the Empty shell and the other two. One of the prominent "features" in these datasets can be seen most readily in Fig 3-9 as the set of peaks between $\left(2.4 \mathrm{msec}, 130^{\circ}\right)$ and $\left(2.8 \mathrm{msec}, 160^{\circ}\right)$. This is the residue of the direct source pulse that has been subtracted off incompletely. It is present to some degree in all datasets in this forward scatter region.

Finally, Figs. 3-10 and 3-11 show the scaled raw scattered return from the Empty and Complex shells respectively for a $5^{\circ}$ incident pulse. This is again a plot of receivers from $0^{\circ}$ to $360^{\circ}$ by $6^{\circ}$. The forward scatter has moved to $185^{\circ}$. This dataset is very nearly identical to the $0^{\circ}$ incident angle data.

\subsubsection{Degree Subarray}

In order to process only the waves of interest, it is necessary to identify the portions

of the receiving array which observe those processes. As discussed by Corrado [5], the compressional waves traveling on the shell approximately phase match into the water via Snell's law:

$$
\theta \approx \cos ^{-1}\left(\frac{c_{0}}{c}\right)
$$

where $c_{0}$ is the water wave speed and $c$ is the speed of the structural wave in question. He estimated the compressional wave speed, $c_{p} \approx 5270 \mathrm{~m} / \mathrm{s}$ and the shear wave speed, $c_{s} \approx 3120 \mathrm{~m} / \mathrm{s}$. The sound speed of the water in the NRL tank was reported at $c_{0}=1488.5 \mathrm{~m} / \mathrm{s}$ for all experiments. This gives a range of angles at which these waves are expected to leave the shell: $73.6^{\circ} \leq \theta_{p} \leq 106.4^{\circ}$ for compressional waves 


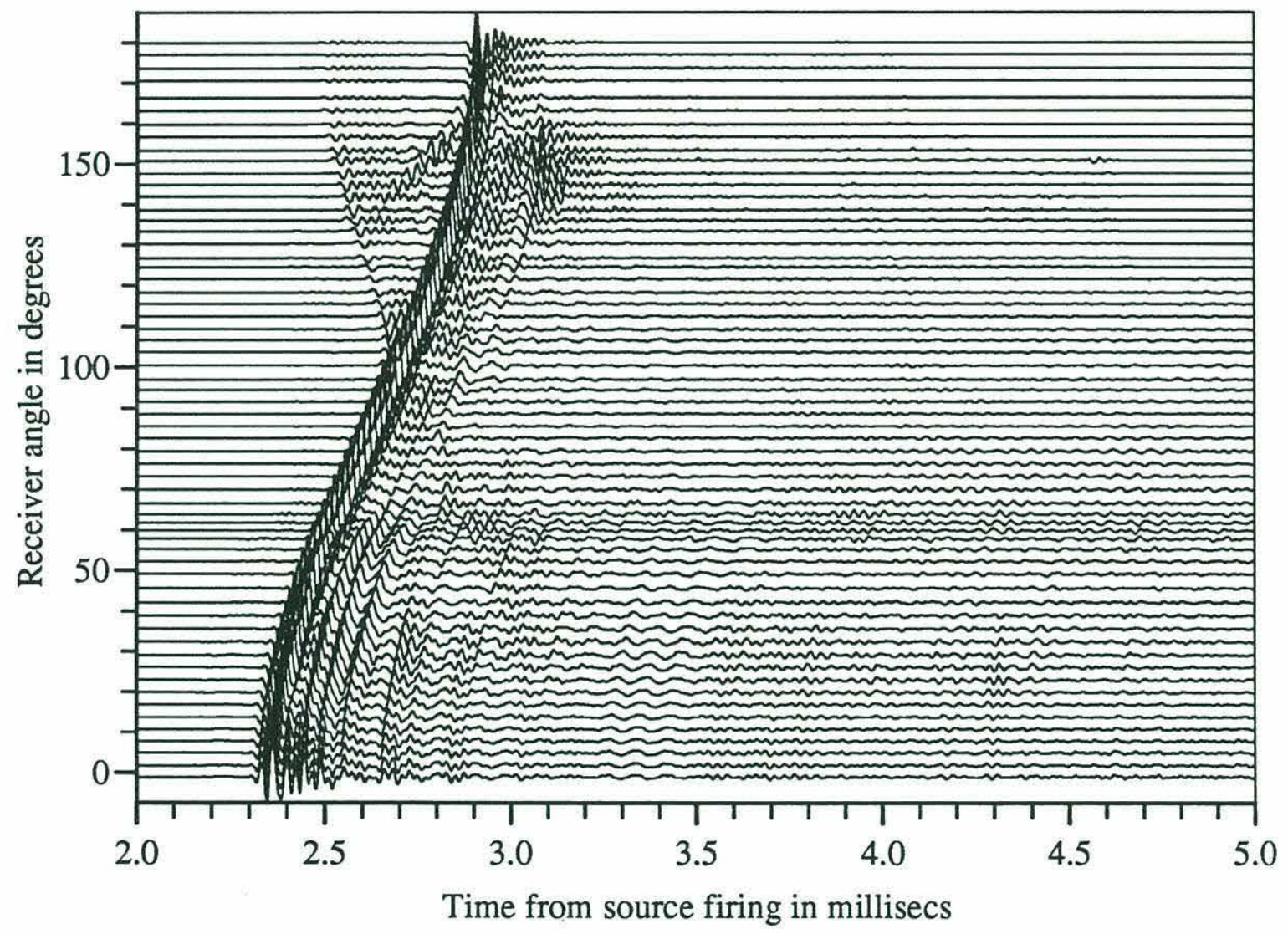

Figure 3-7: Raw data from the Empty shell. Incident angle $=0^{\circ}$. Receivers $0^{\circ}-180^{\circ}$ by $3^{\circ}$. Each line scaled min to max. 




Figure 3-8: Raw data from the Ribbed shell. Incident angle $=0^{\circ}$. Receivers $0^{\circ}-180^{\circ}$ by $3^{\circ}$. Each line scaled min to max. 


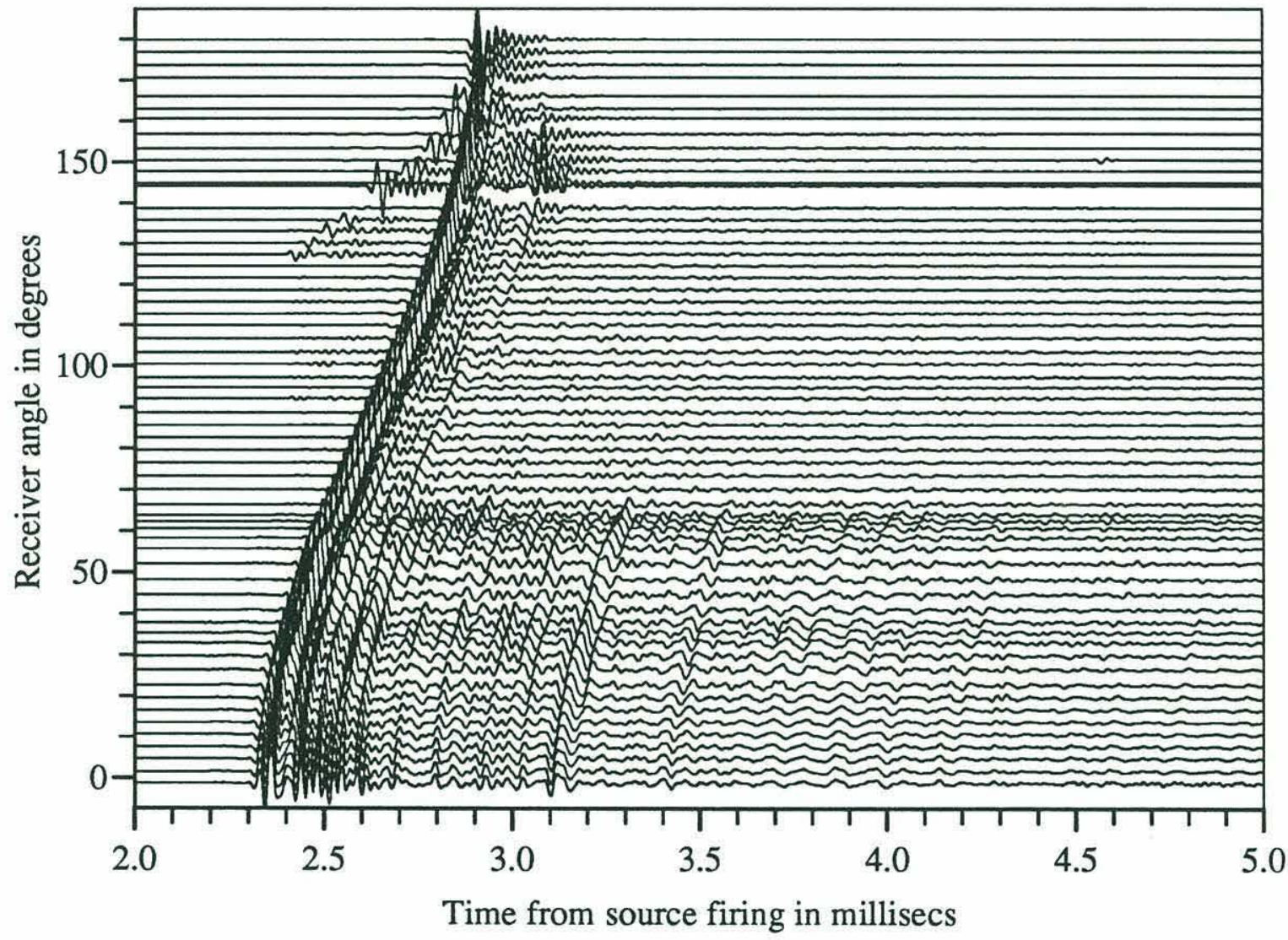

Figure 3-9: Raw data from the Complex shell. Incident angle $=0^{\circ}$. Receivers $0^{\circ}-180^{\circ}$ by $3^{\circ}$. Each line scaled min to max. 


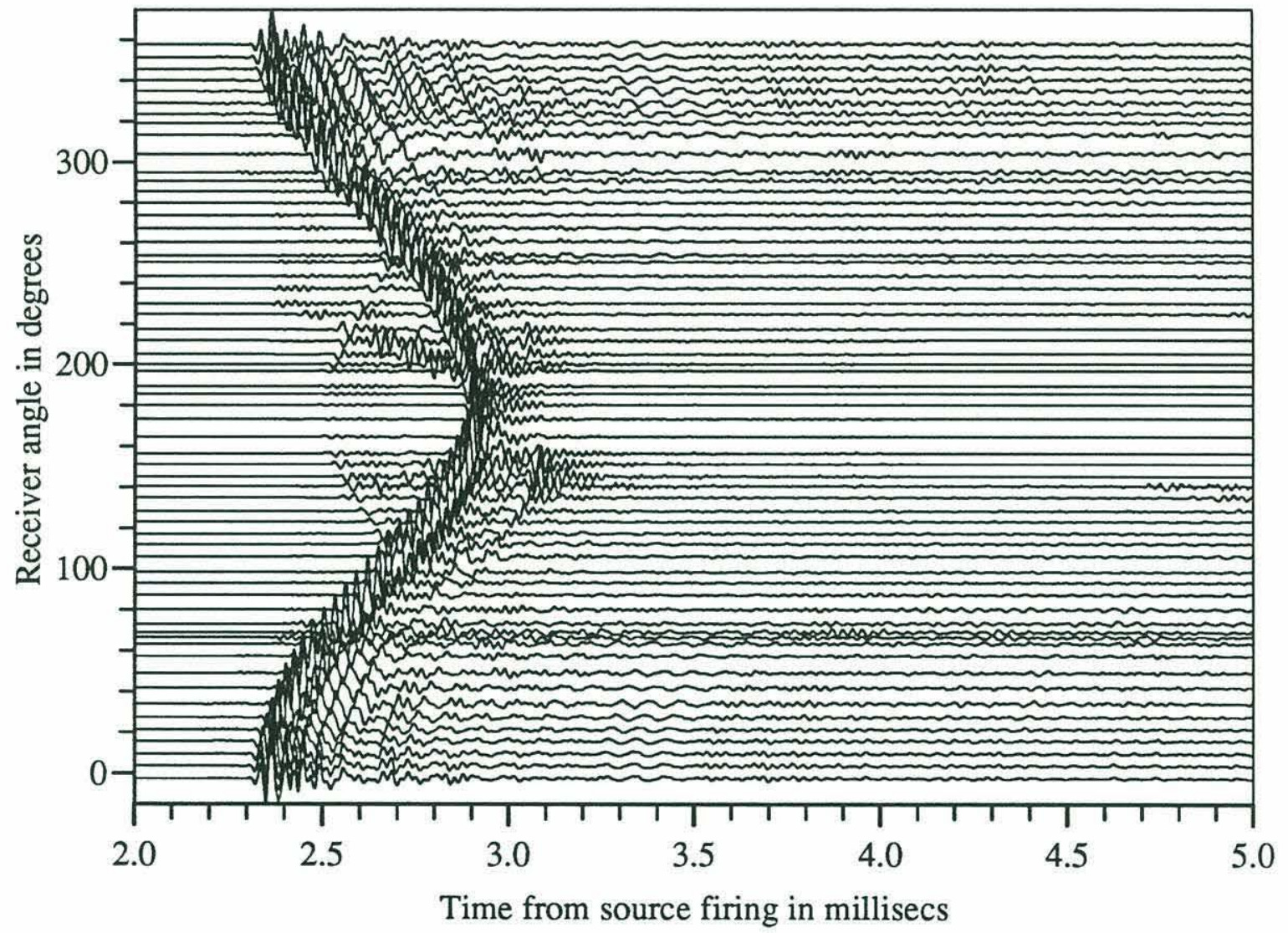

Figure 3-10: Raw data from the Empty shell. Incident angle $=5^{\circ}$. Receivers $0^{\circ}-360^{\circ}$ by $6^{\circ}$. Each line scaled min to max. 




Figure 3-11: Raw data from the Complex shell. Incident angle $=5^{\circ}$. Receivers $0^{\circ}-360^{\circ}$ by $6^{\circ}$. Each line scaled min to max. 
and $61.5^{\circ} \leq \theta_{s} \leq 118.5^{\circ}$ for shear waves, where $0^{\circ}$ is bow and $90^{\circ}$ is beam aspect. Since the waves will leave at this angle all along the shell, this window must be opened slightly. Accounting for this geometry at the array, compressional waves will arrive at receiver elements whose location angle satisfies, $63^{\circ} \leq \theta_{b} \leq 117^{\circ}$ and shear waves will arrive at receiver elements whose location angle satisfies $55^{\circ} \leq \theta_{b} \leq 125^{\circ}$.

Since the waves of interest only occupy a portion of the array, only this part of the array should be used in processing to maintain maximum array gain. Conventional array gain is defined as the ratio of the SNR at a single sensor with a unit level signal to the SNR at the array. For example, if the signal is seen in only one sensor of an $N$ element array, the conventional beamforming operation decreases both the signal and incoherent white noise by a factor of $N$ and array gain equals 1 , but if the signal occupies all $N$ elements, the signal component is maintained and the array gain equals $N$. I chose to process the minimum possible aperture which captures all the waves of interest; this is the range, $60^{\circ} \leq \theta_{b} \leq 120^{\circ}$. All subsequent processing in this thesis is performed on data which comes from this section of the array. Until the description of the processing scheme is completed, I will focus attention on the $0^{\circ}$ incidence case for the Empty and Ribbed shell only. Once the final processing chain is completed, the other incident angles and shell will be re-visited.

To illustrate the problem at hand, it is instructive to look at the raw time series from the 60 degree section of the synthetic array centered around the target beam. Fig. 3-12 and 3-13 show the raw scattered return from the Empty and Ribbed shells respectively at an incident angle of $0^{\circ}$. This time individual line scaling is not required and the full $1^{\circ}$ spacing is shown.

These two plots differ significantly. There is a return spread from $+10^{\circ}$ to $+30^{\circ}$ at $2.65 \mathrm{~ms}$, which turns out to be the diffracted return from the stern endcap. This explanation becomes more obvious as more processing is done. Further, interpretation of these plots is complicated by several factors. First, this data is dominated by a geometrically scattered return from the bow endcap. Also, the source pulse is long in time, therefore events later in time are masked by the tails of the response of earlier events. Finally, events from many points on the shell are superposed at each receiver. 


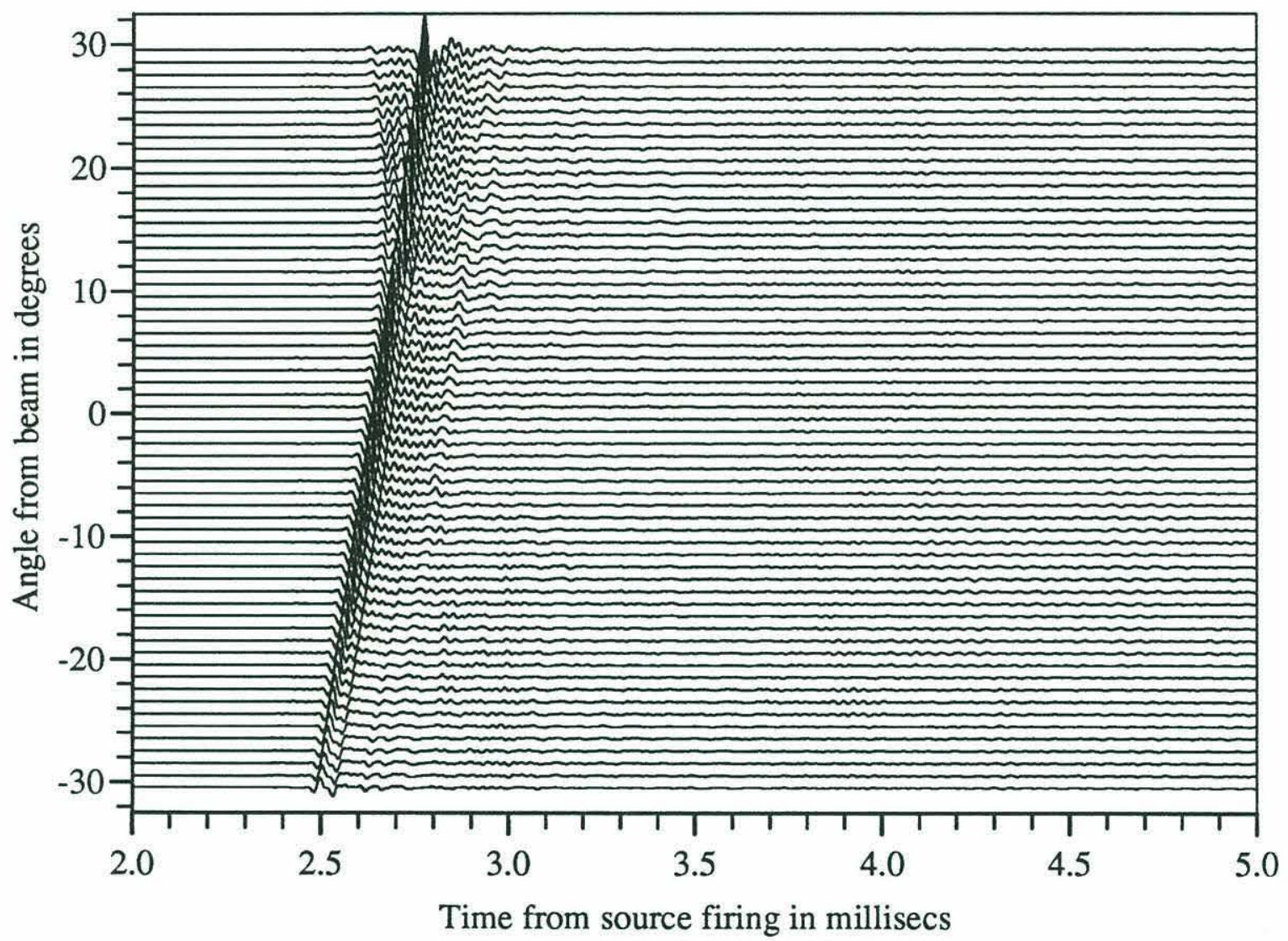

Figure 3-12: Raw data from $60^{\circ}$ of the array by $1^{\circ}$ around shell beam. Empty shell at $0^{\circ}$ incidence. 


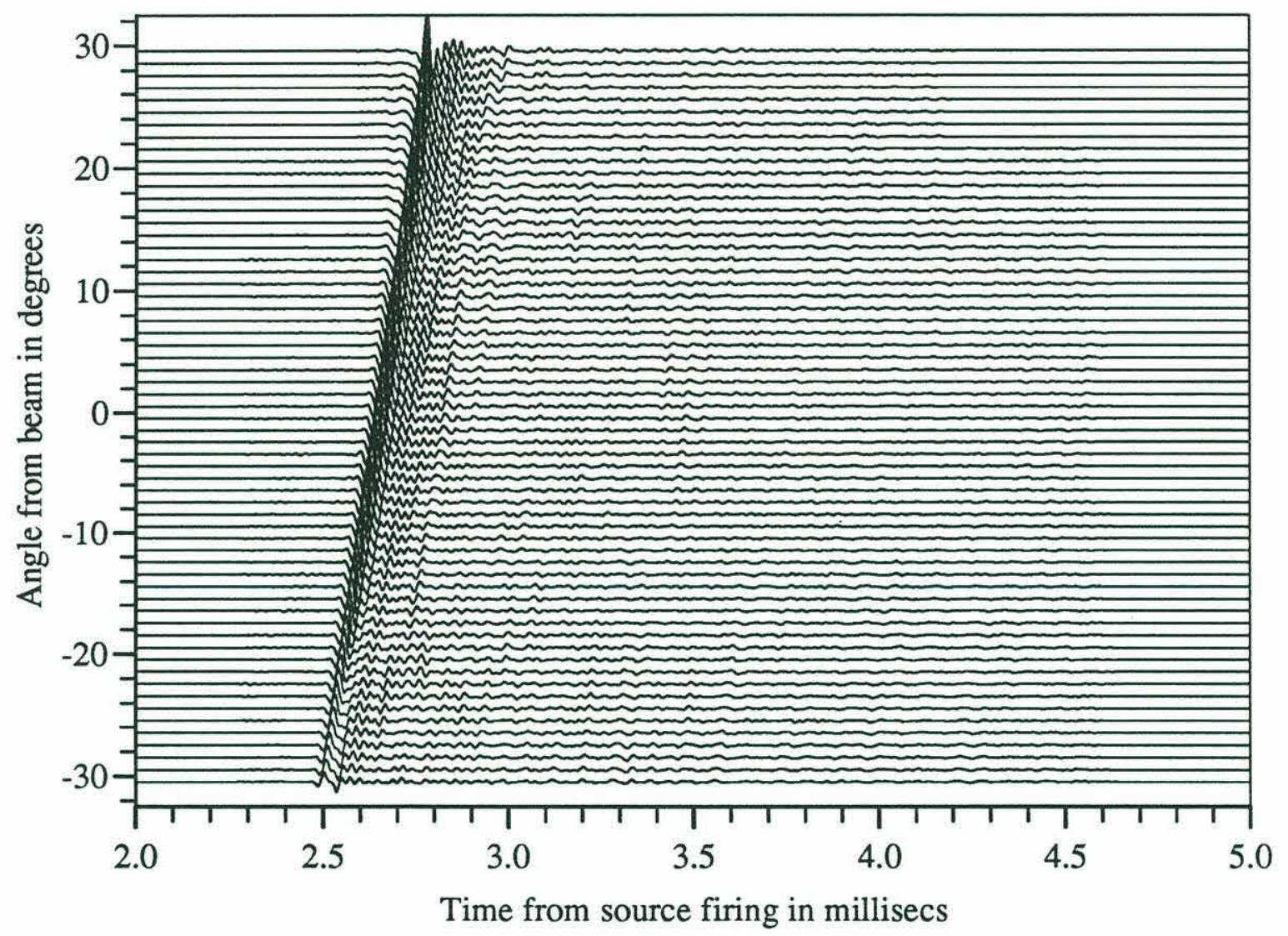

Figure 3-13: Raw data from $60^{\circ}$ of the array by $1^{\circ}$ around shell beam. Ribbed shell at $0^{\circ}$ incidence. 
These effects must be removed one by one to allow quantitative comparison.

\subsection{Deconvolution with Source Pulse}

\subsubsection{Process}

The first step in enhancing the raw time series is deconvolution by the source pulse to improve time resolution. The source pulse was measured for each dataset by placing the receiver at $0.18 \mathrm{~m}$ from the target center (target removed) and measuring the direct field from the source firing. This reference pulse was provide for each shell. The propagation delay of $1.344 \mathrm{msec}$ and the spreading loss of $1 / \sqrt{2 m}=1.414$ were removed to give an estimate of the pulse at the source array. The source pulse for the Empty shell experiments and its spectrum are shown in Fig. 3-14. Recall that data was sampled at $500 \mathrm{khz}$. The source pulse is fairly long in time, about $350 \mu \mathrm{sec}$ and
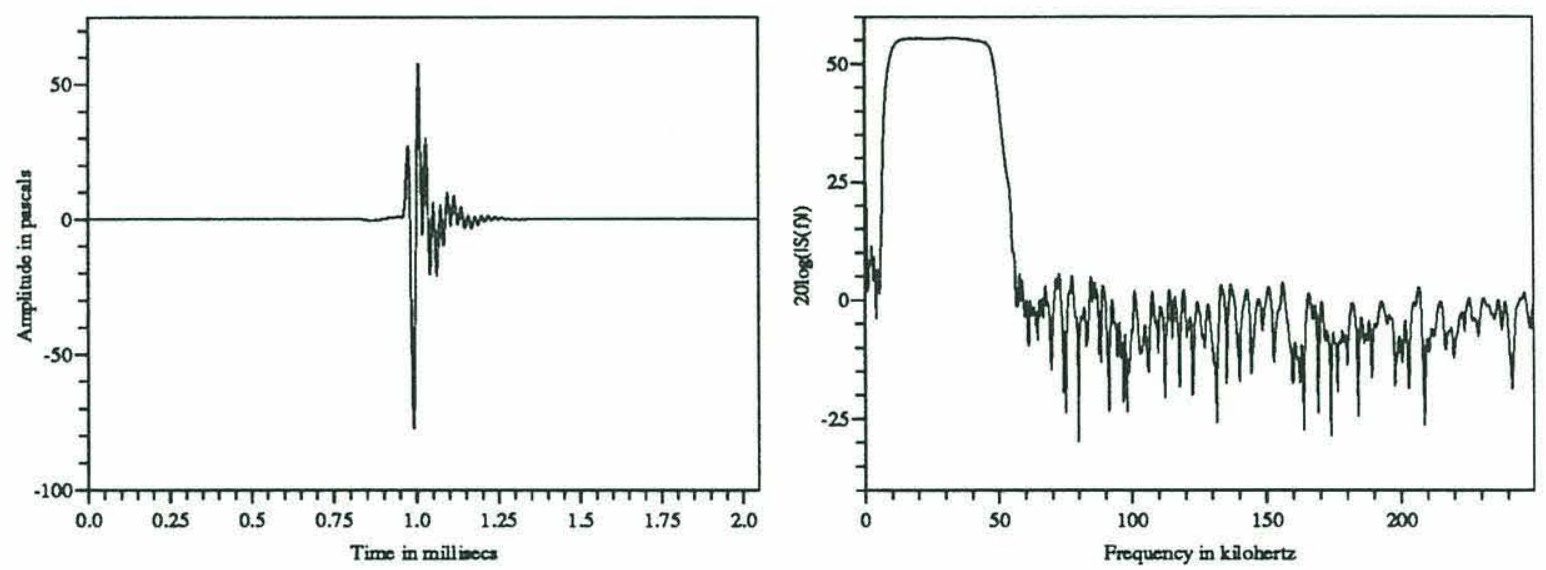

Figure 3-14: (a) Source pulse and (b) Source spectrum.

the energy is concentrated in the band $10.0 k h z<f<47.4 k h z$ or $2.3<k a<11.0$ (3dB down points).

In order to reduce the sidelobes of the source pulse in time, a matched filtering approach was used. The source pulse was deconvolved out of all data, by dividing its frequency response from that of the data. The frequency domain was then windowed with a Hamming window and transformed back to the time domain. The resulting effective source pulse is shown in Fig. 3-15. 

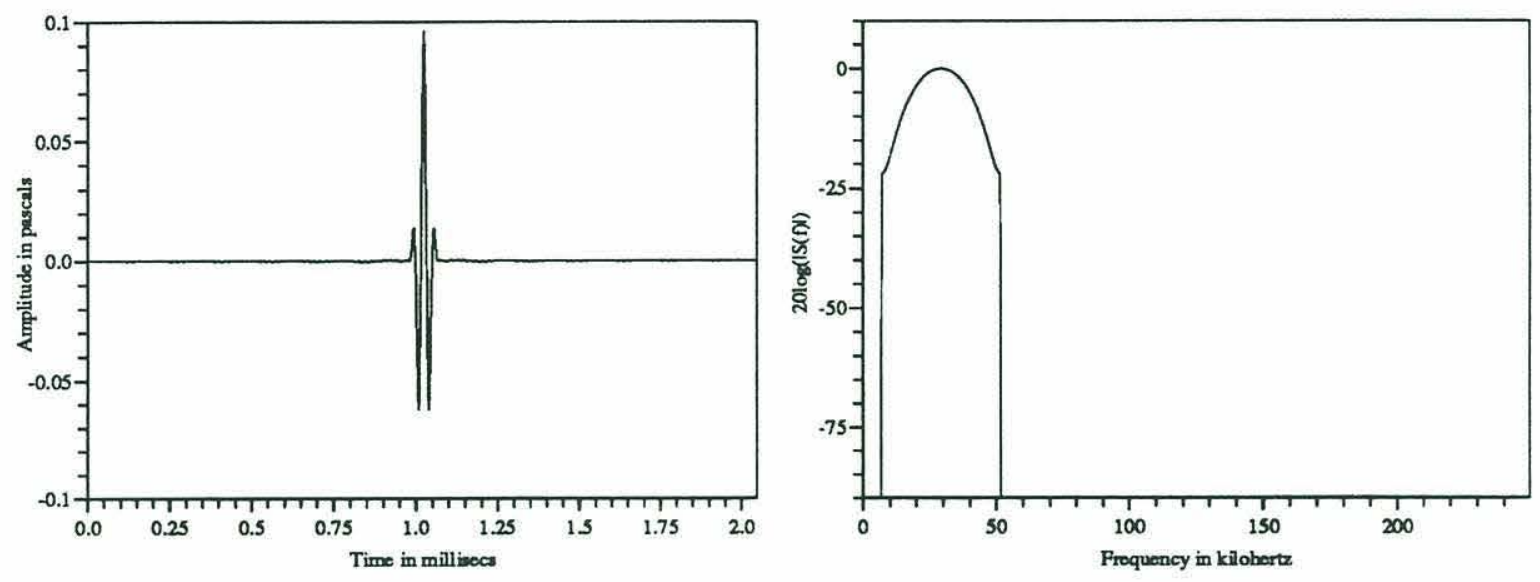

Figure 3-15: (a) Hamming source pulse and (b) Hamming source spectrum.

The Hamming window is $22 d B$ down at its endpoints. Therefore it was applied to the data at the $22 d B$ down points of the source pulse, occurring at $k a=1.65$ and $k a=12.04$. The applied Hamming window gives an effective $(3 d B)$ beamwidth of $4.91<k a<8.79$ The remaining frequency values outside of the Hamming window were zeroed. This results in a compact source pulse about $160 \mu \mathrm{sec}$ wide with $42 d B$ sidelobes. All source pulses are similar and were therefore processed according to this same scheme.

\subsubsection{Results}

The raw data from a $60^{\circ}$ arc of the array around the shell beam, deconvolved by source pulse for the Empty and Ribbed shells at $0^{\circ}$ incidence is shown in Figs. 3-16 and 3-17 respectively. Compare these plots to Figs. 3-12 and 3-13. Note that the peaks in the time traces are now much more compact in time. This compacting of signal energy reveals a second return clearly present $200 \mu \mathrm{sec}$ after the initial return over the entire angular range. This is the first evidence of flexural wave energy on the shell. Why this is true will become clear in subsequent processing. 


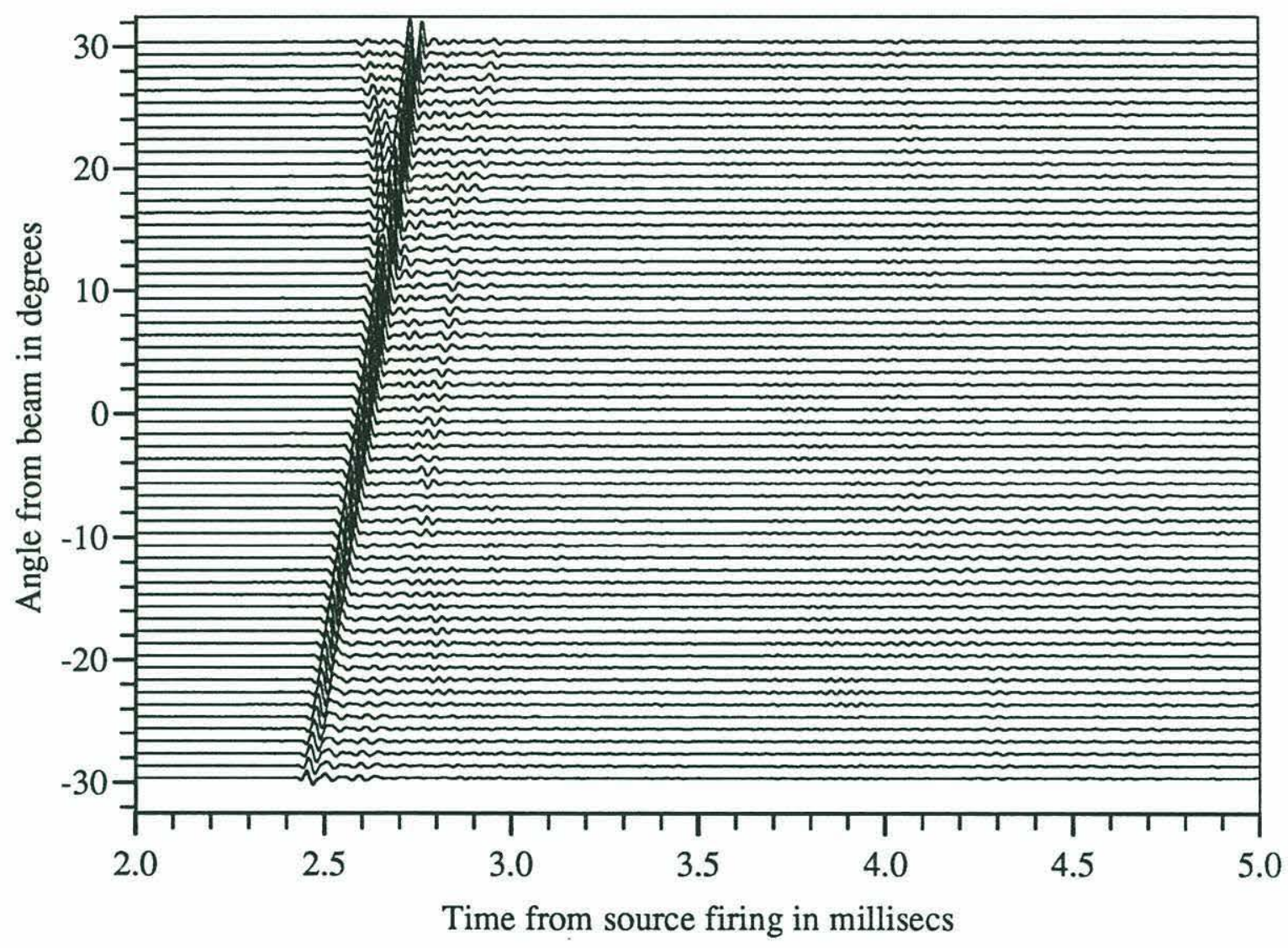

Figure 3-16: Raw data from $60^{\circ}$ of the array by $1^{\circ}$ around shell beam, deconvolved by source pulse. Empty shell at $0^{\circ}$ incidence. 


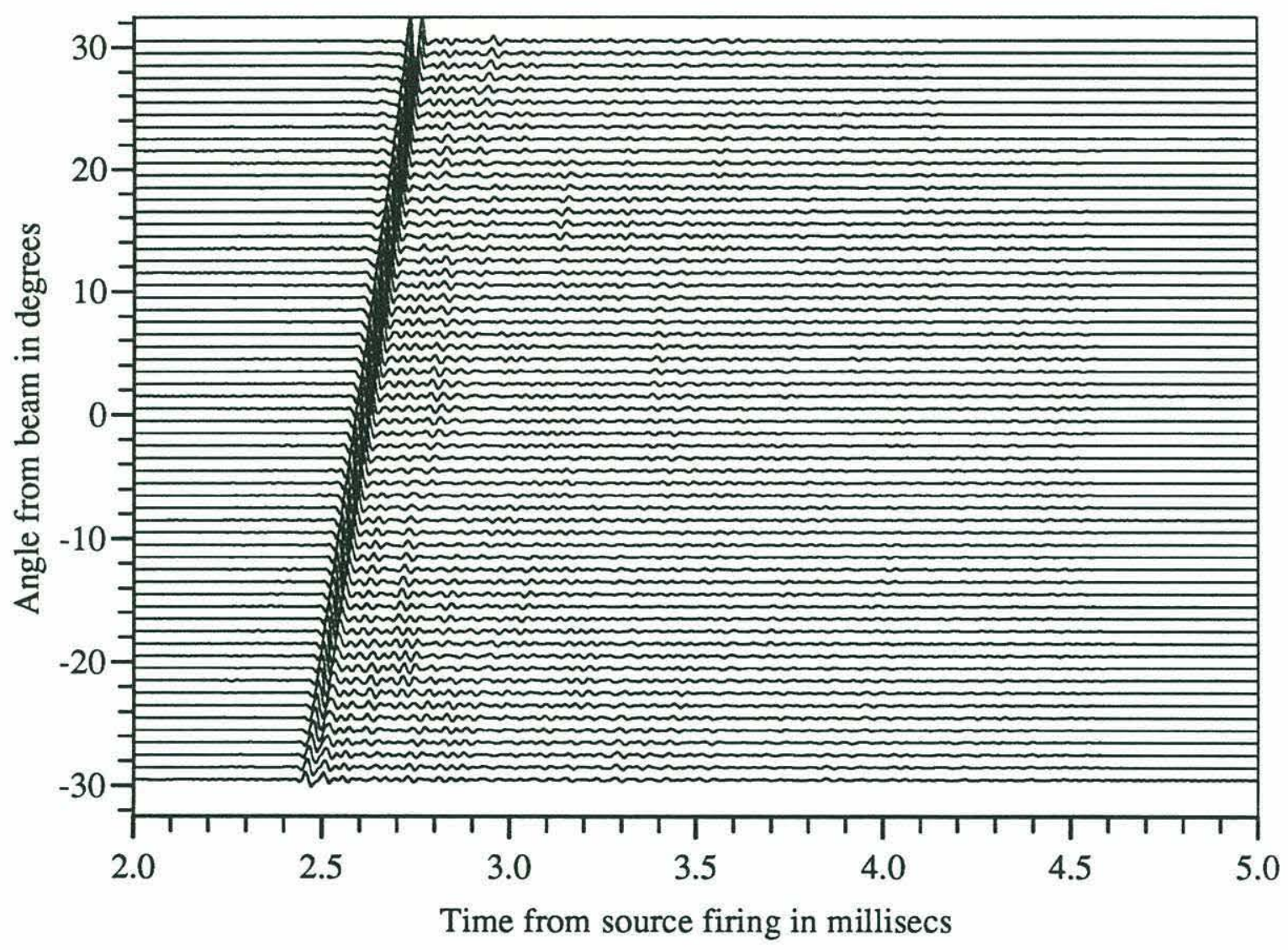

Figure 3-17: Raw data from $60^{\circ}$ of the array by $1^{\circ}$ around shell beam, deconvolved by source pulse. Ribbed shell at $0^{\circ}$ incidence. 


\subsection{Conventional Focusing Results}

\subsubsection{Rectangular array weighting}

The next step in the processing chain is to remove the propagation to the receiver array and look at the response on the surface of the shell itself. This is accomplished using the conventional focusing technique described in Section 2.3. The 61 array elements are focused to 51 points in space corresponding to equally spaced samples on the surface of the shell closest to the collection array.

The result of focusing the $60^{\circ}$ sub-array of raw data, deconvolved by the source pulse, is shown in Fig. 3-18 and Fig. 3-19 for the Empty and Ribbed shell respectively at $0^{\circ}$ incidence. This is a waterfall plot, in which each trace represents the time response of a virtual "source" on the shell surface which radiates into the far field. The $\mathrm{x}$-axis is time from the line array source firing in milliseconds and the $y$-axis is location on the shell surface along the length of the shell given in centimeters from the shell center. In this scheme, $-43 \mathrm{~cm}$ is the bow of the shell, $43 \mathrm{~cm}$ is the stern.

The focused result is dominated by a large return at $(1.25 \mathrm{sec},-43.0 \mathrm{~cm})$. This is the geometrically scattered return from the bow endcap and its associated sidelobes. This return is nearly identical for both shells. The sidelobe structure is visible as peaks of energy along a line connecting points $(1.25 \mathrm{sec},-43.0 \mathrm{~cm})$ and $(1.55 \mathrm{sec}, 43.0 \mathrm{~cm})$. Notice that this structure extends well past the middle of the shell. These artifacts of the focusing operation obscure the waves of interest. It is important to reduce their contribution.

\subsubsection{Hamming tapered array weighting}

The first process used to reduce the sidelobes of all waves on the shell is adjustment of the array weighting. Although, there is a great deal of research devoted to the weighting functions of arrays, there is no general procedure for arrays which are not equally spaced line arrays. Since the array is equally spaced on the arc of a circle of large radius of curvature, I decided to use the same approach that was used to 


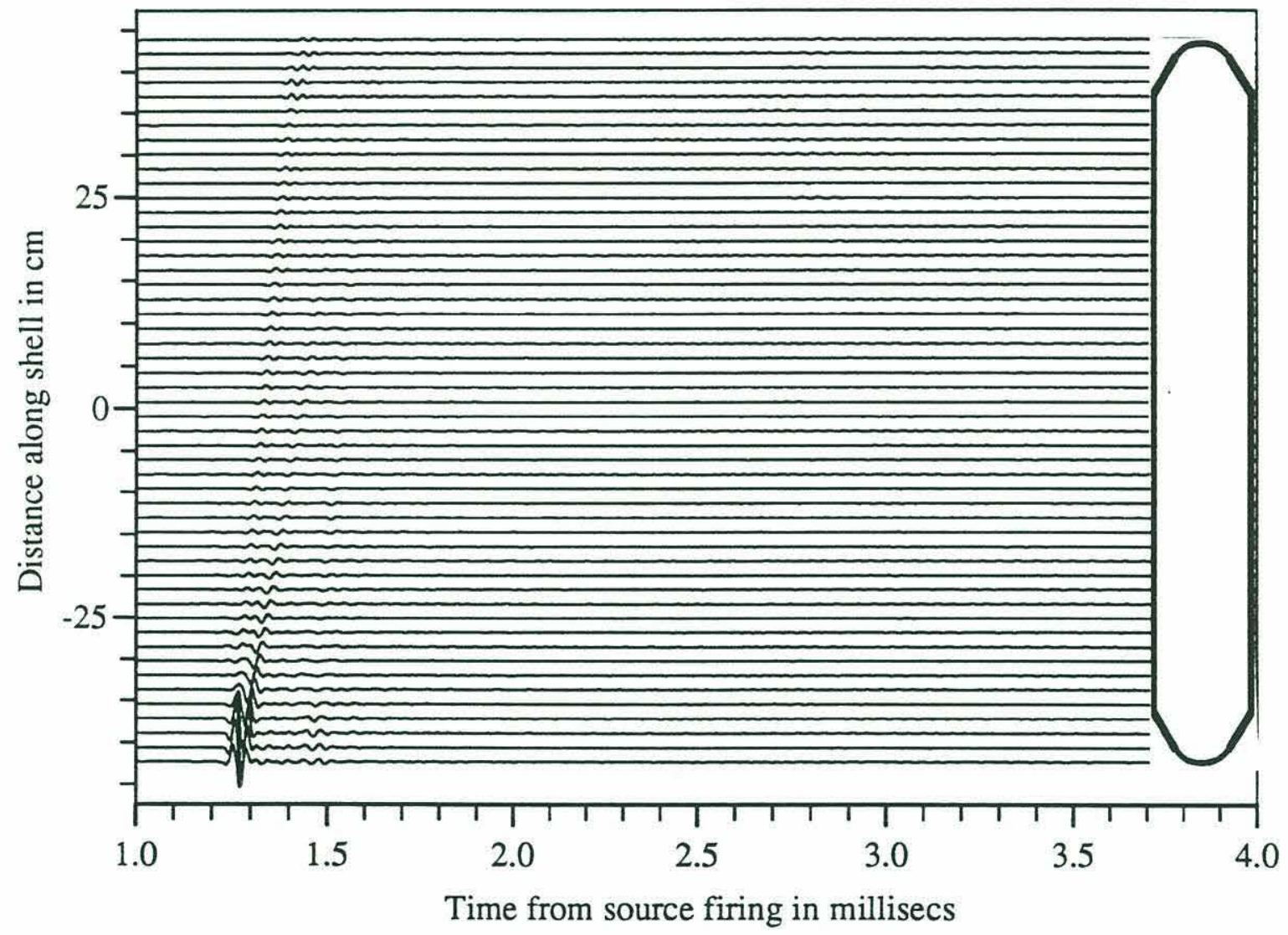

Figure 3-18: $60^{\circ}$ of array around shell beam, deconvolved by the source pulse and focused onto shell. Empty shell data at $0^{\circ}$ incidence. 


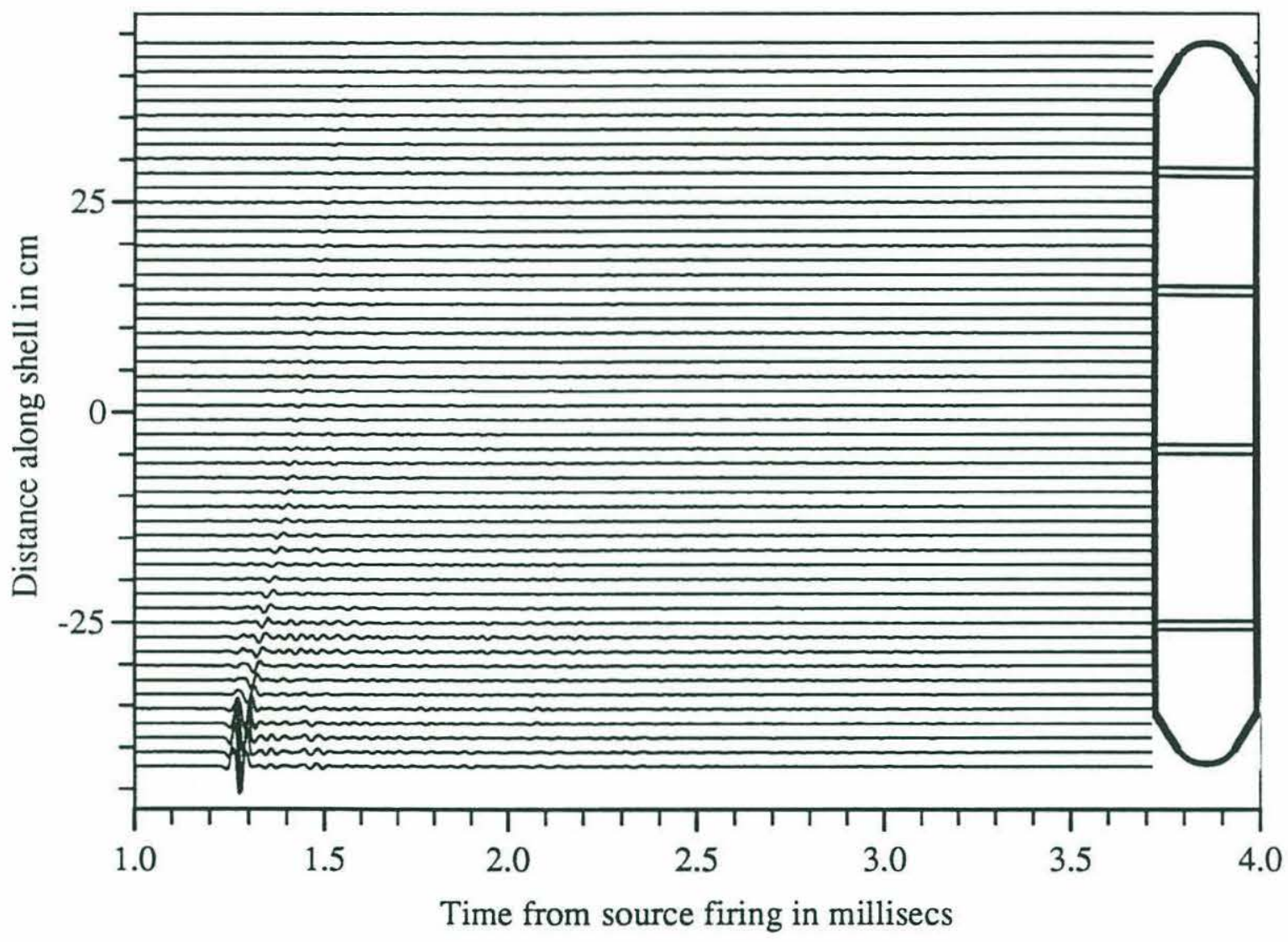

Figure 3-19: $60^{\circ}$ of array around shell beam, deconvolved by the source pulse and focused onto shell. Ribbed shell data at $0^{\circ}$ incidence. 
time window the target-dependent returns from the tank walls. The $60^{\circ}$ of array was weighted by a rectangular window with Hamming ends. The Hamming portion extends for $15^{\circ}$ on either end.

The result of focusing the $60^{\circ}$ sub-array of raw data, deconvolved by the source pulse and windowed with a Hamming tapered window is shown in Fig. 3-20 and Fig. 321 for the Empty and Ribbed shells respectively. Notice that the sidelobes have been significantly reduced. However, the response is still dominated by the geometrically scattered return from the endcap region due to its very high amplitude. For this reason, it became necessary to remove this return by estimating it and subtracting it out of the data.

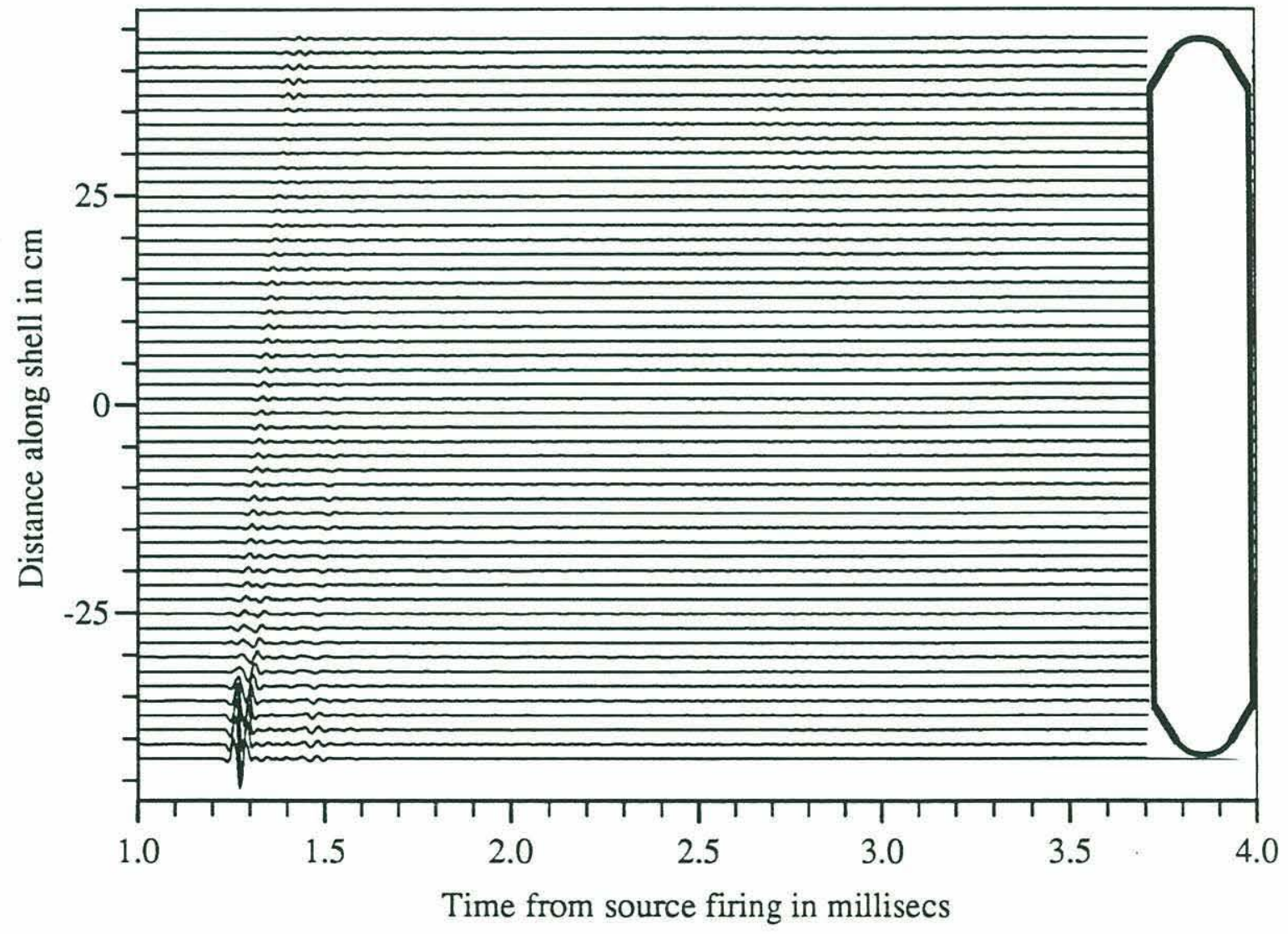

Figure 3-20: $60^{\circ}$ of array around shell beam, deconvolved by the source pulse, Hamming tapered and focused onto shell. Empty shell data at $0^{\circ}$ incidence. 


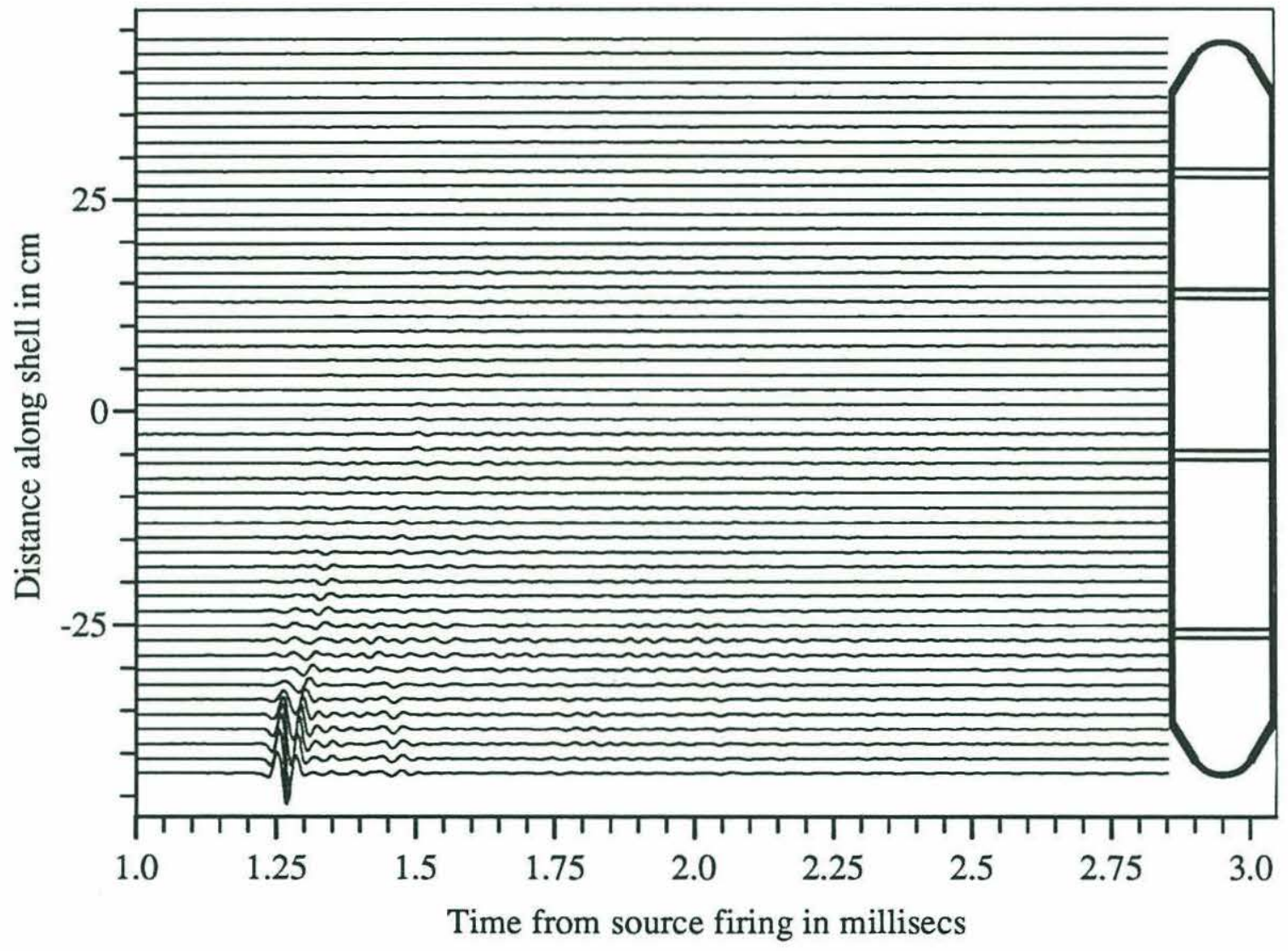

Figure 3-21: $60^{\circ}$ of array around shell beam, deconvolved by the source pulse, Hamming tapered and focused onto shell. Ribbed shell data at $0^{\circ}$ incidence. 


\subsection{Removal of Geometrically Scattered Return}

\subsubsection{Process}

The shell is thin and air filled, so I chose to approximate the geometrically scattered return from the endcap as a return from a pressure release sphere. In this case, the geometrically scattered return is $180^{\circ}$ out of phase with the incident pulse and modified in amplitude as a function of receiver angle. Conti et al. [27] show that this is a good approximation for the initial segment of the return.

In order to remove this wave, the process diagrammed in Fig. 3-22 was followed. The time series from each receiver were correlated with the source reference pulse. The

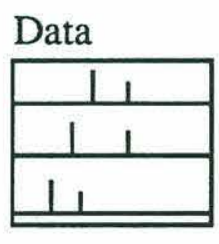

Source Pulse

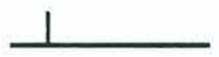

Amplitude and

Delay Estimates

$\mathrm{a}_{1}, \mathbf{t}_{1}$

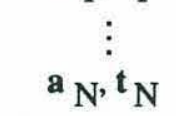

Source Pulse



Data

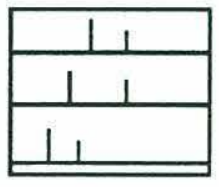

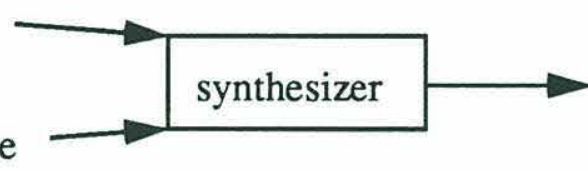

Synthetic Geometric-only

Data

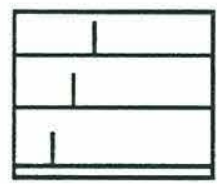

Amplitude and

Delay Estimates

$a_{1}, t_{1}$

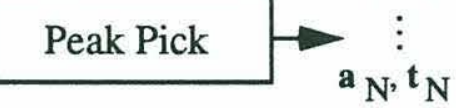

Synthetic

Geometric-only

Data

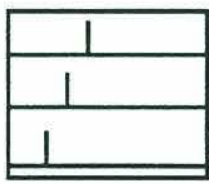

Geometric

Removed

Data

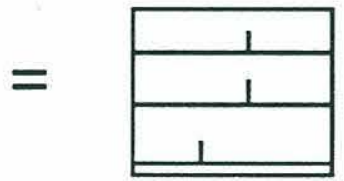

Figure 3-22: Block diagram of process used to remove geometrically scattered return.

time location of the peak of each correlation is assumed to be the arrival time of the geometrically scattered return and the amplitude of this peak, $P$, to be proportional 
to its amplitude, $a$. The amplitude is:

$$
a=P / \sum_{t} s^{2}(t)
$$

where $s(t)$ is the reference pulse. Once the arrival time and amplitude were estimated for each receiver, the return itself was approximated by the source pulse delayed by the estimated delay and multiplied by the estimated amplitude of each receiver. This dataset containing only the estimated geometrically scattered return was subtracted from the raw dataset before any other processing. Focusing then provides array gain to reduce the geometrically scattered return by an additional $10 \mathrm{~dB}$.

\subsubsection{Results}

As a byproduct, this processing provides an estimate of the beampattern of the geometrically scattered return. Fig. 3-23 shows the amplitude estimate of the geometrically scattered return as a function of receiver angle for the three shells for the $0^{\circ}$ incidence case. This plot includes receiver angles which are $\pm 90^{\circ}$ around the shell beam or receivers at $0^{\circ}-180^{\circ}$. The bold line is for the Empty shell, the plain line for the Ribbed shell and the dotted line is for the Complex shell.

Fig. 3-24 shows the amplitude estimate of the geometrically scattered return as a function of receiver angle for the three shells for the $75^{\circ}$ incidence case. This plot includes receiver angles which are $\pm 90^{\circ}$ around the shell beam or receivers at $-105^{\circ}-75^{\circ}$. This is the backscattered regime.

Notice that for both incidence angles the amplitude of the geometrically scattered component does not vary appreciably from shell to shell, validating the assumption it "looks like" the source pulse at any incidence angle. Small discontinuities in these plots are due to incorrect identification of the peak corresponding to the geometrically scattered return in the correlation. These always lie outside the range of receivers used for focusing and thus do not corrupt any of the processing shown in this thesis.

This completes the processing chain which implements the direct analysis leg of the Hybrid Processing Structure and forms the basis for the following physical analysis of 


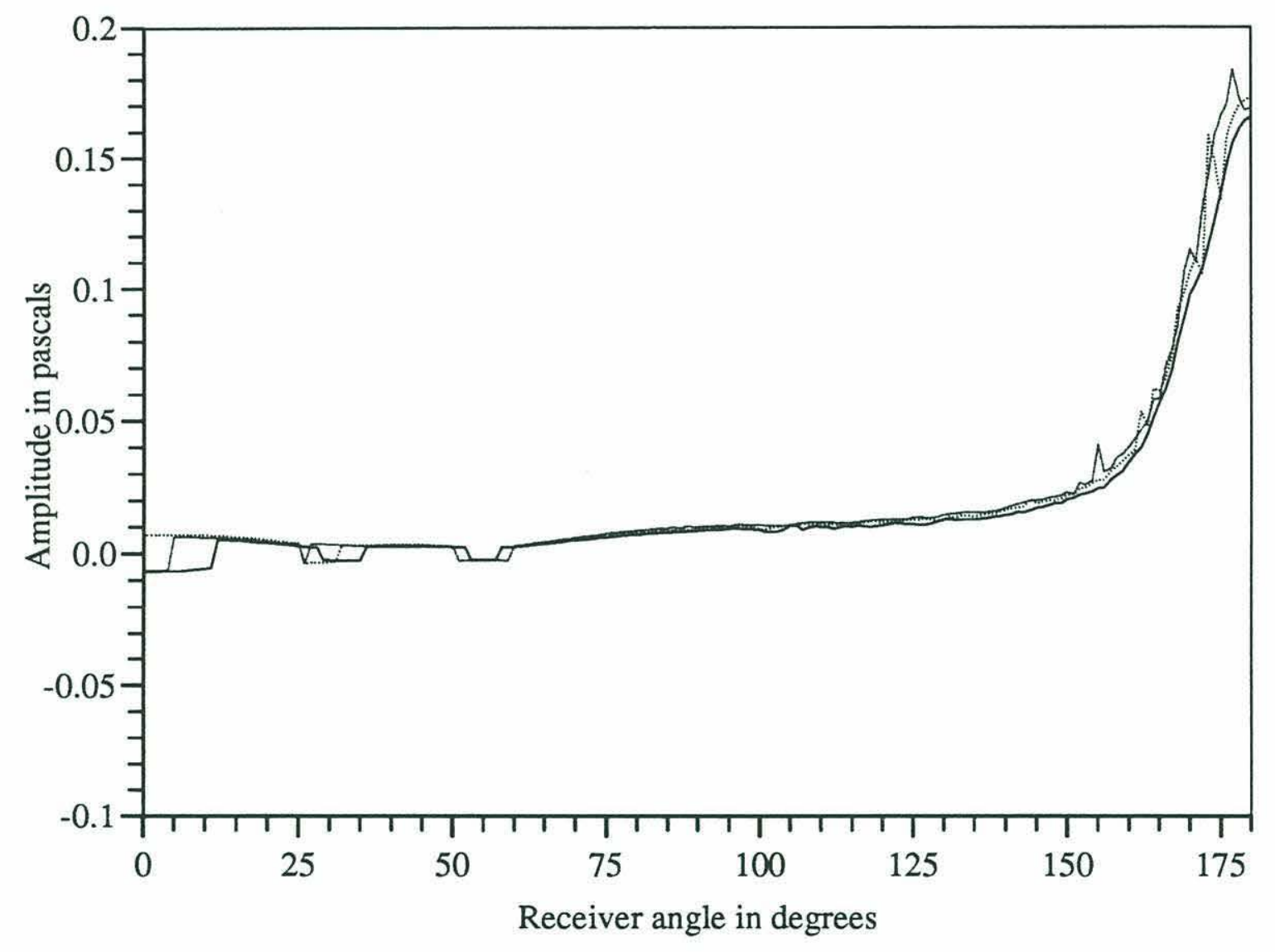

Figure 3-23: Amplitude estimate for the geometrically scattered return from the Empty (bold), Ribbed (plain) and Complex (dotted) shells at $0^{\circ}$ incidence. 


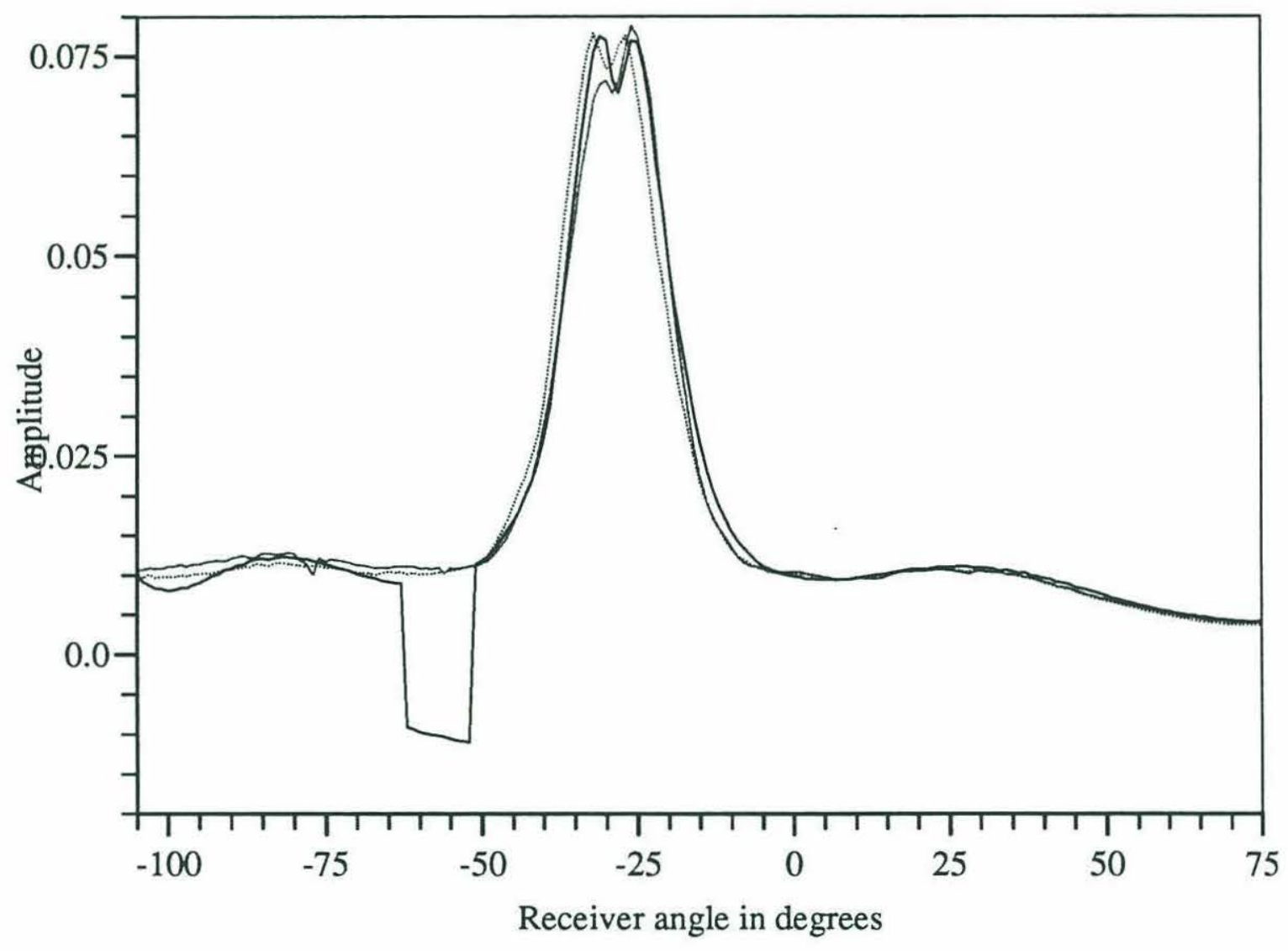

Figure 3-24: Amplitude estimate for the geometrically scattered return from the Empty (bold), Ribbed (plain) and Complex (dotted) shells at $75^{\circ}$ incidence. 
the shells. A block diagram of the complete direct analysis processing chain is shown in Fig. 3-25. This chain was applied to all the datasets shown in Section 3.1.1.

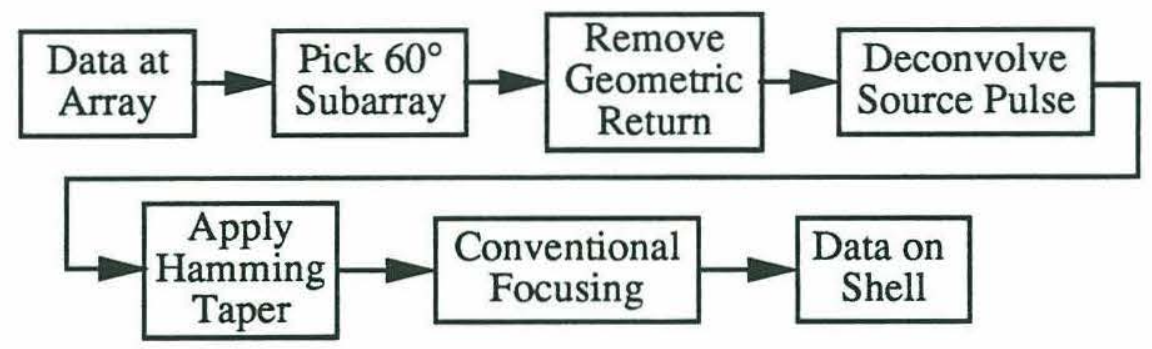

Figure 3-25: Block diagram of complete direct analysis processing chain.

\subsection{Results of Processing Chain for All Data}

The geometrically scattered return removed, source pulse deconvolved, spatial Hamming tapered, conventionally focused data is shown in Fig. 3-26, 3-27 and 3-28 for the Empty, Ribbed and Complex shells respectively for the $0^{\circ}$ incidence case.

The differences among the three shells become immediately obvious. The Empty shell plot shows a compressional wave coupling onto the shell at $(1.25 \mathrm{msec},-43.0 \mathrm{~cm})$. The moveout of this wave matches the compressional speed estimated by Corrado [5] of $5270 \mathrm{~m} / \mathrm{sec}$. The wave propagates to the other end of the shell where there is another large burst of energy from the stern endcap. This stern endcap return can be traced back to the raw data of Fig. 3-12 as the early arrival at $(2.6 \mathrm{msec}, 30 \mathrm{deg})$. This return is noticeably absent in the Ribbed and Complex shell raw data. The focused data of Fig. 3-27 shows that the first compressional wave in the Ribbed shell propagates to the stern with a serious energy reduction due to reflection by the rib stiffeners. Note that the ring stiffeners are located at $-27.62 \mathrm{~cm},-4.13 \mathrm{~cm}$, $14.87 \mathrm{~cm}$, and $27.62 \mathrm{~cm}$ and the shell-endcap discontinuities are located at $\pm 36.94 \mathrm{~cm}$. The compressional waves in the Ribbed and Complex shells are bouncing back and forth in the bays between the ring stiffeners and in the bays between the endcap and rings. Notice that these waterfall plots have a horizontally banded structure defined by these bays and that the energy in these bays decreases down the shell length. 


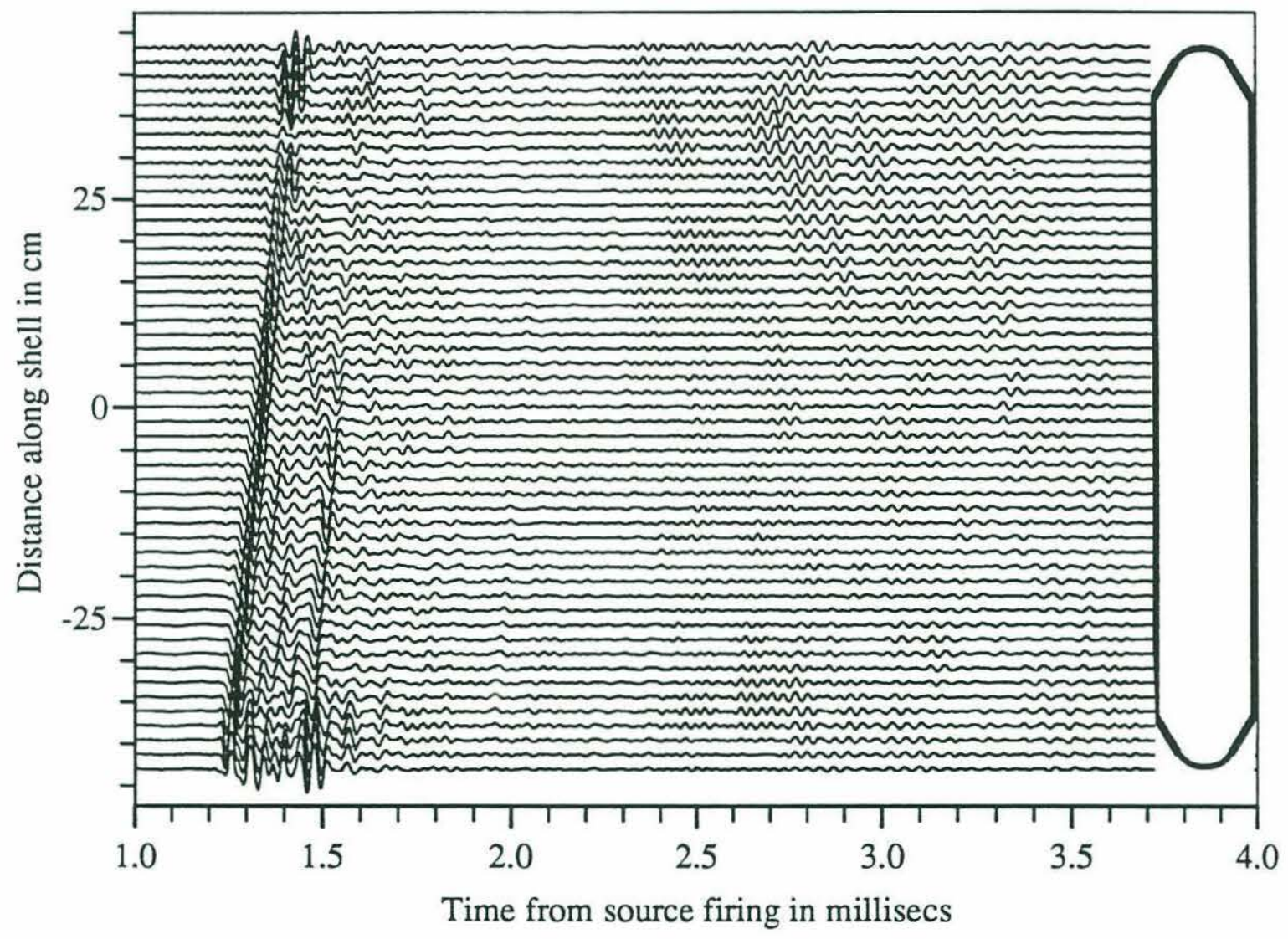

Figure 3-26: $60^{\circ}$ of array around shell beam, geometrically scattered return removed, deconvolved by the source pulse, Hamming tapered and focused onto shell. Empty shell data at $0^{\circ}$ incidence. 


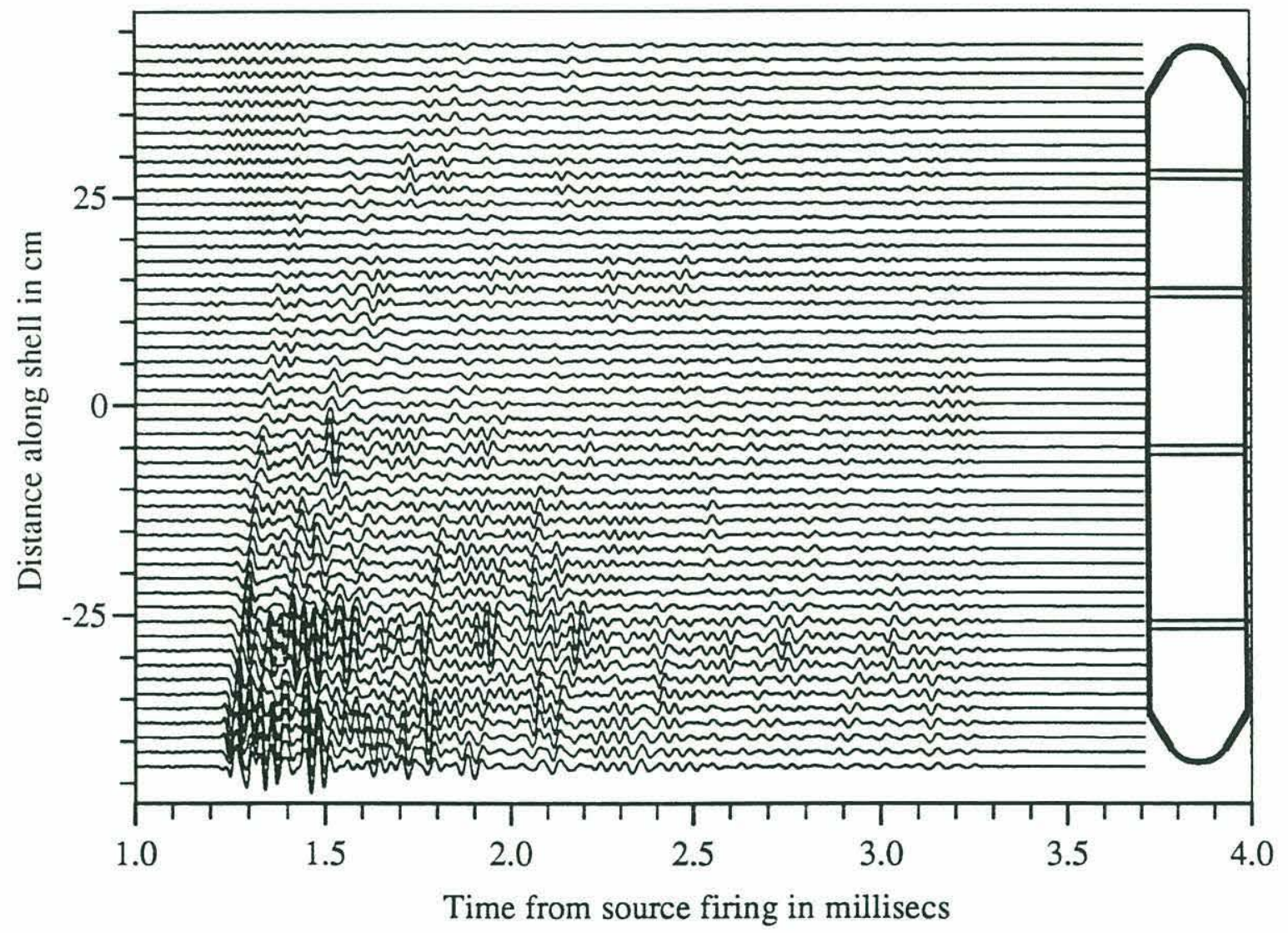

Figure 3-27: $60^{\circ}$ of array around shell beam, geometrically scattered return removed, deconvolved by the source pulse, Hamming tapered and focused onto shell. Ribbed shell data at $0^{\circ}$ incidence. 


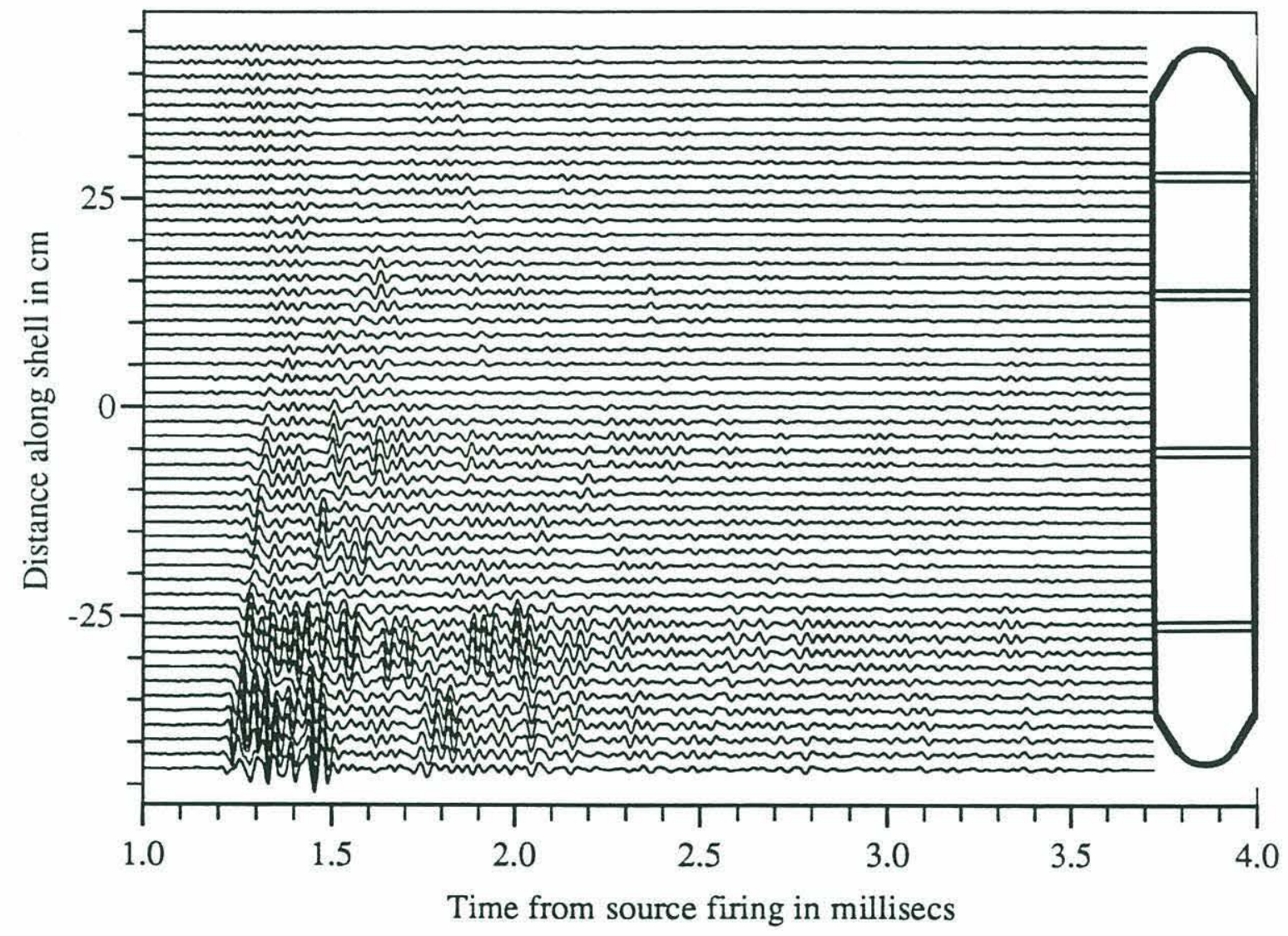

Figure 3-28: $60^{\circ}$ of array around shell beam, geometrically scattered return removed, deconvolved by the source pulse, Hamming tapered and focused onto shell. Complex shell data at $0^{\circ}$ incidence. 
At this incident angle of $0^{\circ}$, these shells can only support symmetric modes, only 0th order flexural and compressional modes should be generated. A feature of the Empty shell data in Fig. 3-26 is the indirect evidence of flexural waves traveling on the shell. The flexural waves on these cylindrical shells are dispersive with wave speeds in the range $300 \mathrm{~m} / \mathrm{sec}-600 \mathrm{~m} / \mathrm{sec}$ and are, therefore, subsonic with respect to the water. Is is only possible to measure them at the array when they couple to other radiating wave types, in this case compressional waves. A second compressional wave which propagates the length of the shell can be seen $200 \mu \mathrm{sec}$ after the initial compressional wave. This return is due to flexural waves induced in the endcap by the incident pulse that have been converted to compressional waves by the endcap-shell discontinuity. There is another flexural wave which couples to compressional waves in the Empty shell data of Fig. $3-26$ at $(2.4 \mathrm{msec}, 36 \mathrm{~cm})$. The slow non-radiating flexural wave has traveled down the shell and coupled to fast, radiating compressional waves at the shell-endcap discontinuity. The flexural wave is dispersive, so the wave packet has been smeared out by this time. This is also true at the bow endcap later in time, compressional waves at the stern endcap have coupled into flexural waves, traveled back to the bow and coupled back to compressional. This is a weaker process due to the extra bounce, but is still visible.

The Ribbed and Complex shell data also show the flexural induced return at the bow end $200 \mu \mathrm{sec}$ after the initial compressional wave, but not cleanly. The flexural and compressional waves are coupling with each other at each discontinuity and ring stiffener in a very complicated manner. The late time flexural coupling at the stern endcap is also present but attenuated.

The geometrically scattered return removed, source pulse deconvolved, spatial Hamming tapered, conventionally focused data is shown in Fig. 3-29 and 3-30 for the Empty and Complex shells respectively for the $5^{\circ}$ incidence case. Notice that this angle of incidence is nearly identical to the $0^{\circ}$ incidence case. Work by Conti and Dyer [28] shows that only the symmetric modes are important and that they remain dominant for angles within $20^{\circ}$ of axial incidence. The noticeable difference between the two incidence angles is the late compressional wave at $3.7 \mathrm{msec}$. This appears to 
be an experimental artifact. The cleaning window for this incidence angle was longer and therefore did not exclude a return, most probably from the bottom of the tank. This small anomaly can be seen in the raw data of Fig. 3-11 at $\left(4.8 m s e c .140^{\circ}\right)$.

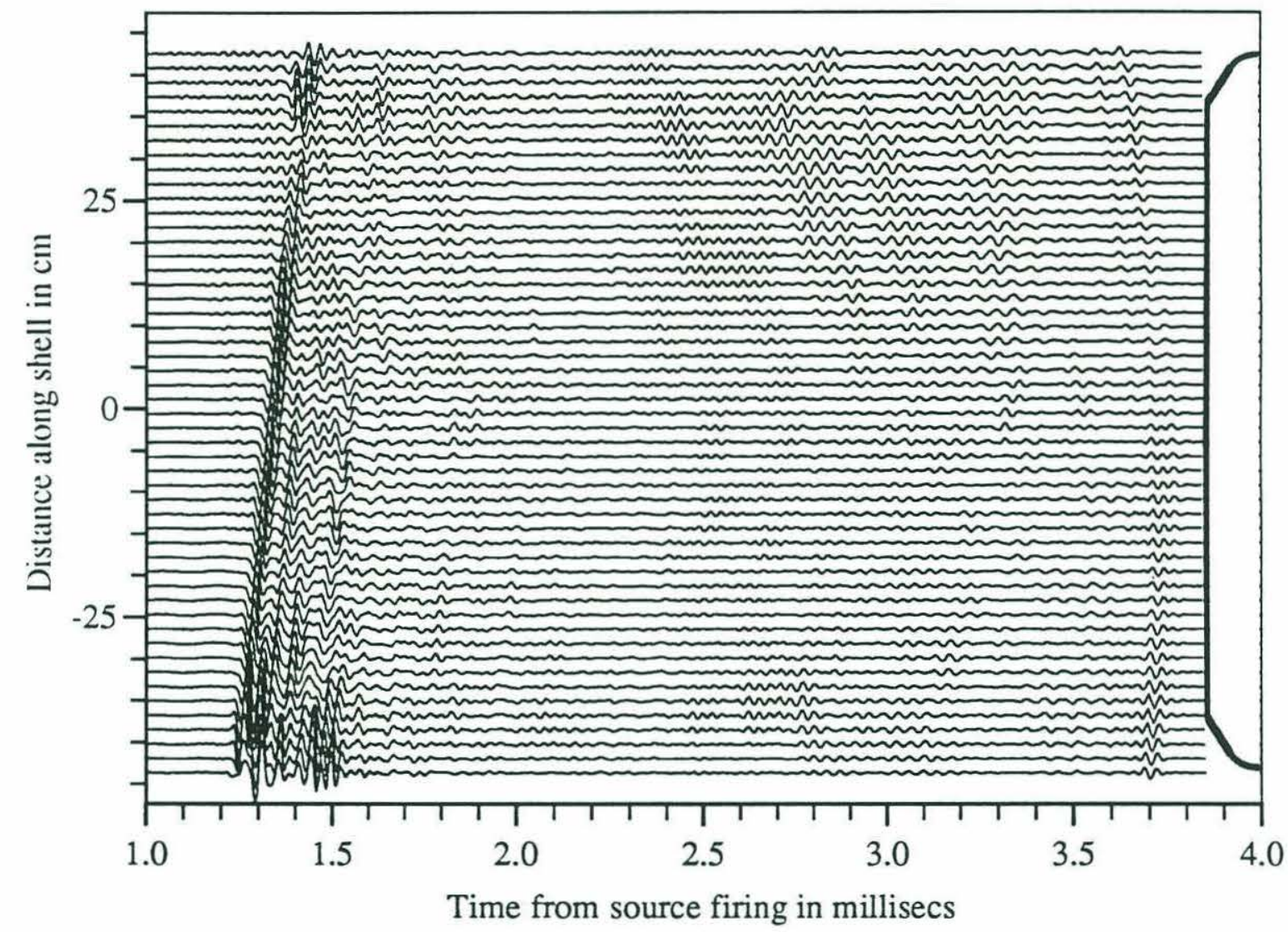

Figure 3-29: $60^{\circ}$ of array around shell beam, geometrically scattered return removed, deconvolved by the source pulse, Hamming tapered and focused onto shell. Empty shell data at $5^{\circ}$ incidence.

Similarly processed waterfall plots are shown in Figs. 3-31, 3-32 and 3-33 for the Empty, Ribbed and Complex shells for the $75^{\circ}$ incidence case. At this angle of incidence both shear and compressional waves are excited by phase matching. This is no longer a single mode case, many circumferencial modes are excited causing helical waves to travel around and down the shell. The collection array has no extent circumferencially around the shell. Therefore, it cannot completely measure the field at this incident angle. The spatial aliasing in the circumferencial direction makes quantitative identification of modes impossible. Fig. 3-31 shows that the incident 


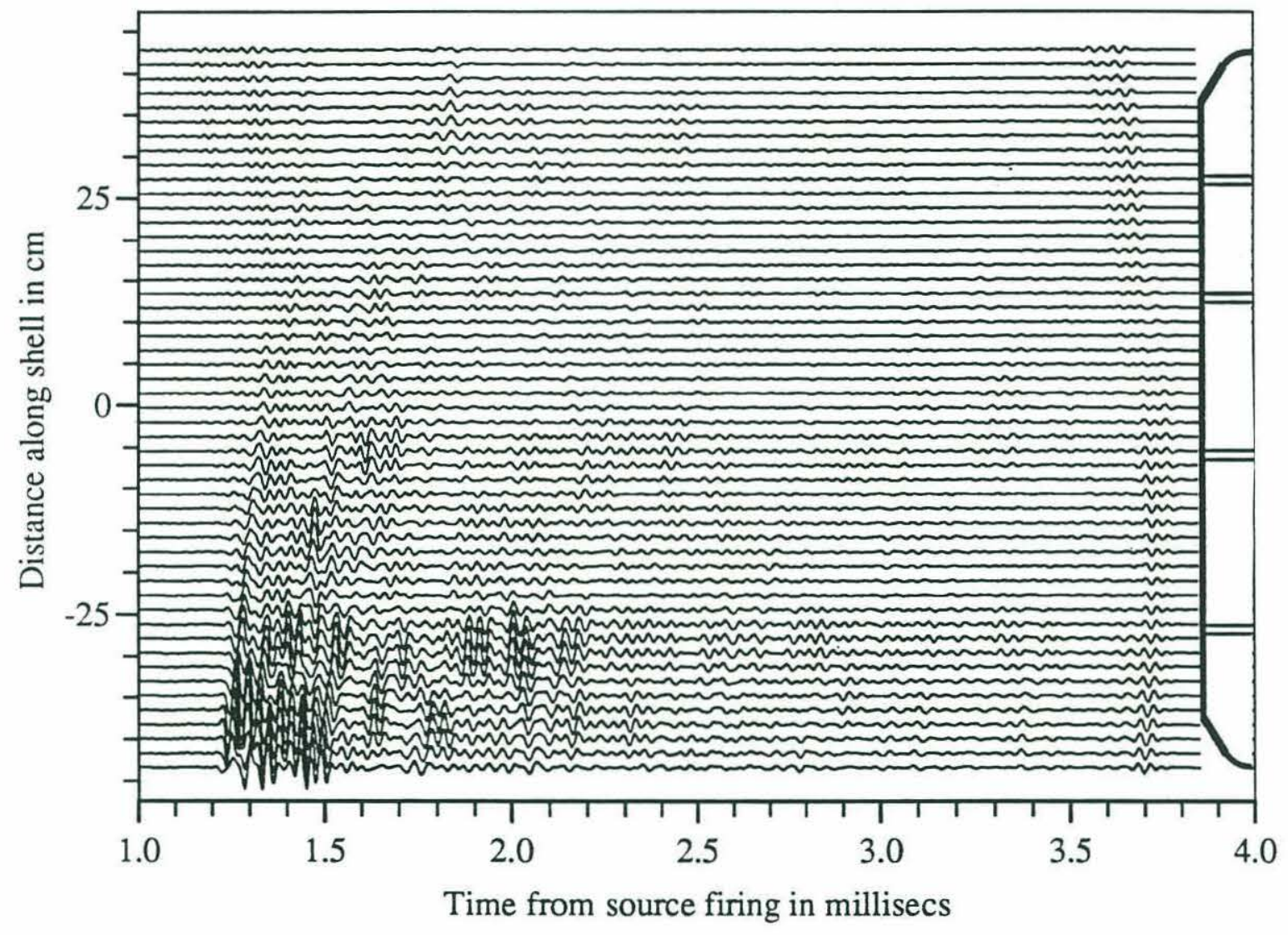

Figure 3-30: $60^{\circ}$ of array around shell beam, geometrically scattered return removed, deconvolved by the source pulse, Hamming tapered and focused onto shell. Complex shell data at $5^{\circ}$ incidence. 
pulse couples onto the shell along its entire length. The pulse travels around the shell several times, radiating as it goes. This is the set of periodic returns. There is also an axial group delay associated with this propagation. The energy propagates to the bow, reflects towards the stern etc. This helical wave is only visible when radiating towards the collection array.

The Ribbed and Complex shells do not have a clean structure as does the Empty shell. As in the $0^{\circ}$ case, the ribs are causing reflection and coupling between wavetypes, which results in a very complicated spatial pattern. An interesting result is the fact that the response of the Complex shell seems lower late in time than that for the Ribbed shell. This will be quantified in the energy measurements in the next section.

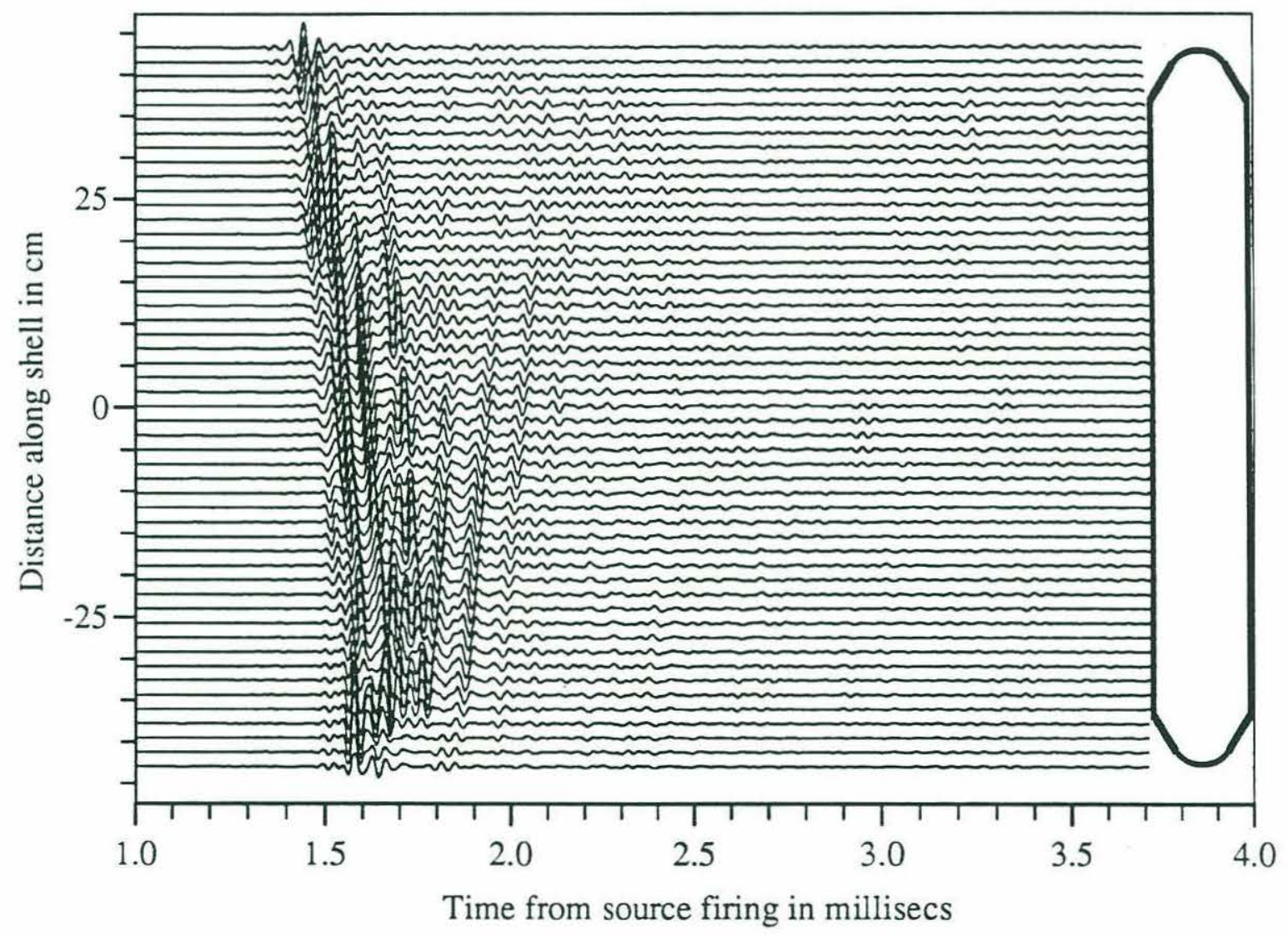

Figure 3-31: $60^{\circ}$ of array around shell beam, geometrically scattered return removed, deconvolved by the source pulse, Hamming tapered and focused onto shell. Empty shell data at $75^{\circ}$ incidence.

A similarly processed waterfall plot is shown in Fig. 3-34 for the Complex shell for 


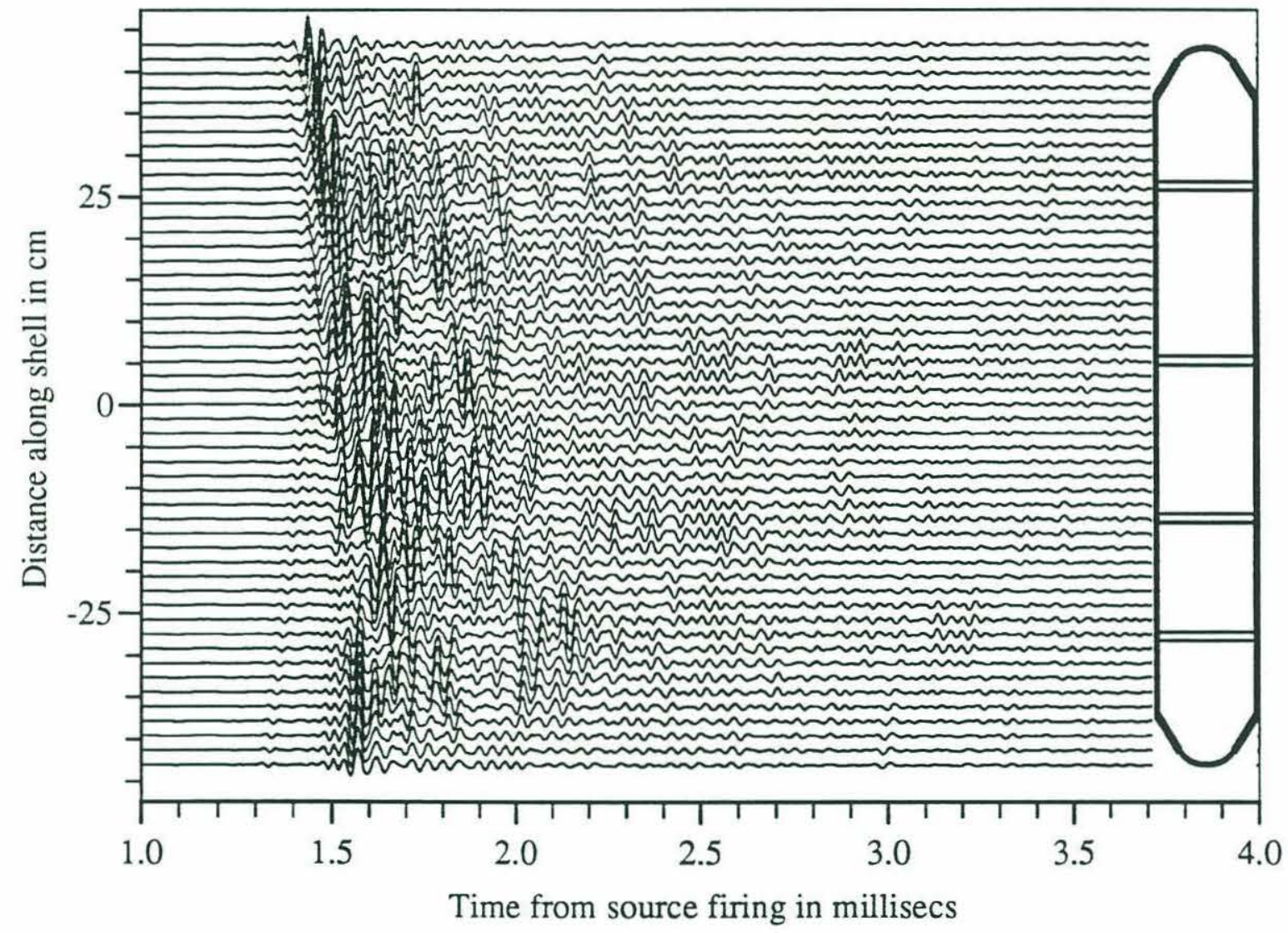

Figure 3-32: $60^{\circ}$ of array around shell beam, geometrically scattered return removed. deconvolved by the source pulse, Hamming tapered and focused onto shell. Ribbed shell data at $75^{\circ}$ incidence. 


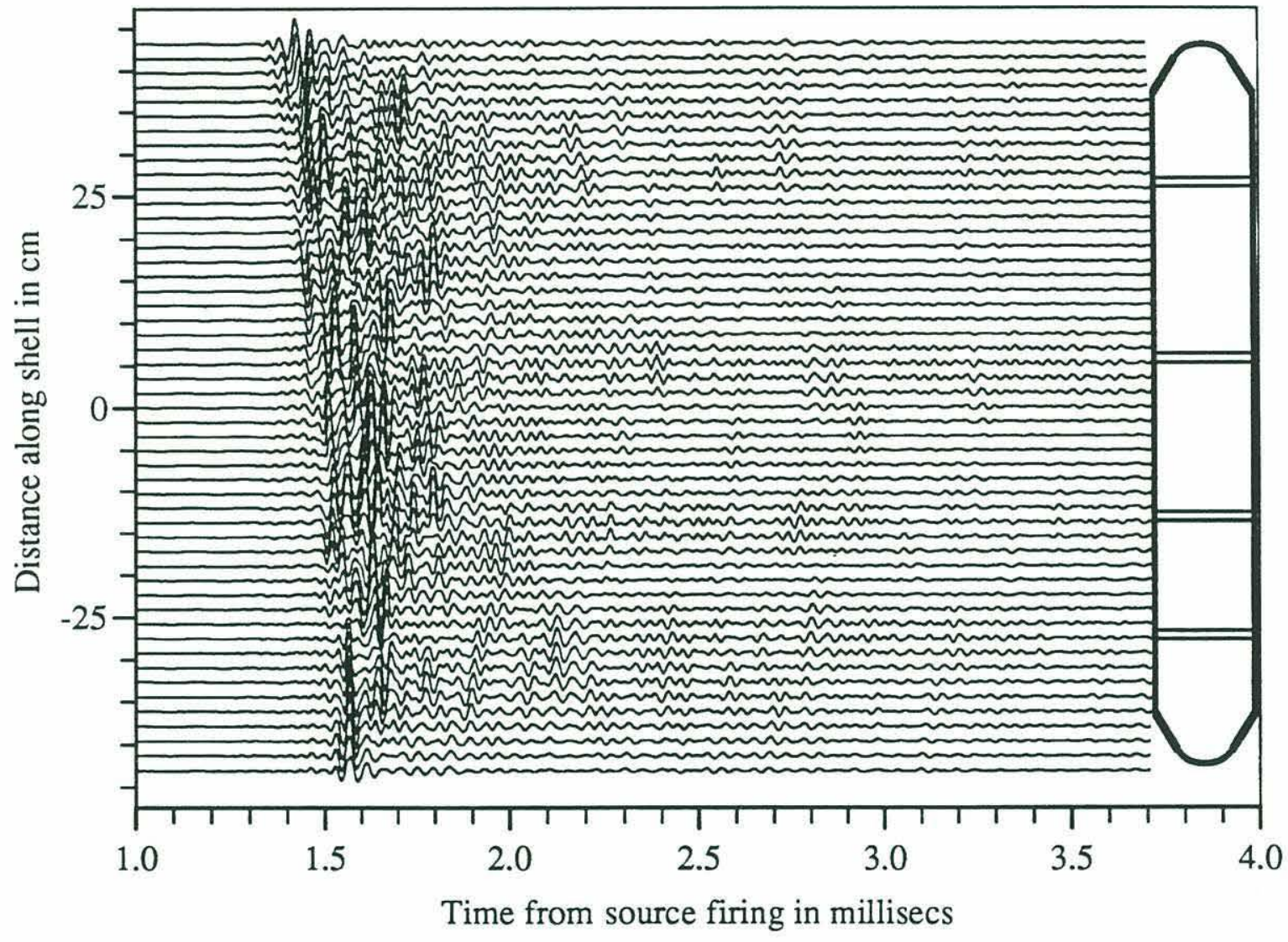

Figure 3-33: $60^{\circ}$ of array around shell beam, geometrically scattered return removed, deconvolved by the source pulse, Hamming tapered and focused onto shell. Complex shell data at $75^{\circ}$ incidence. 
the $66^{\circ}$ incidence case. Here only shear waves should be directly excited on the shell by the source pulse. Again coupling occurs all along the shell and the ribs break up the spatial coherence of the traveling waves. The energy levels at this incident angle are comparable to those at $75^{\circ}$ incidence. This will be quantified in the next section and shows that shear waves are the dominant phenomena at near beam incidence.

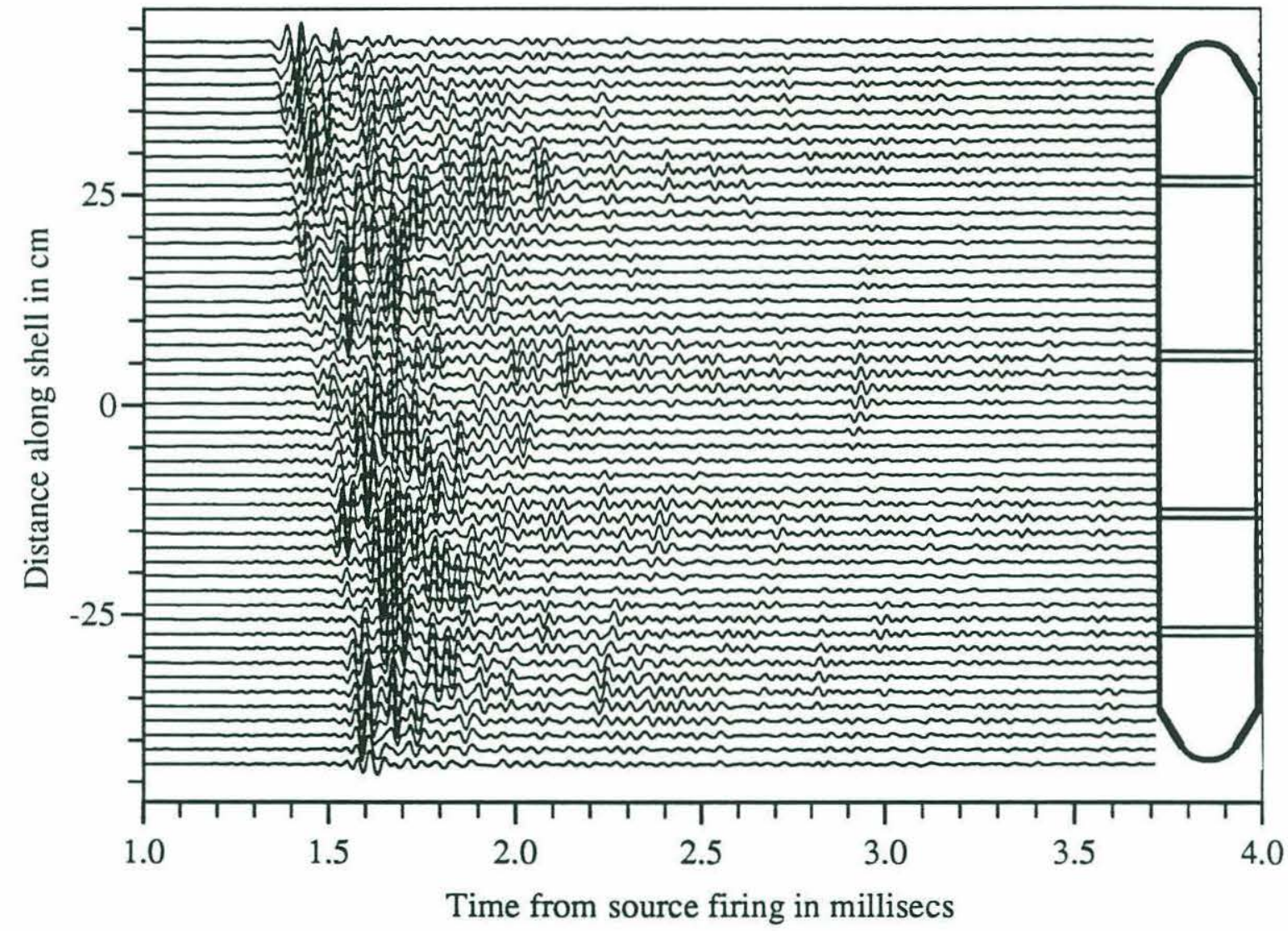

Figure 3-34: $60^{\circ}$ of array around shell beam, geometrically scattered return removed, deconvolved by the source pulse, Hamming tapered and focused onto shell. Complex shell data at $66^{\circ}$ incidence.

A similarly processed waterfall plot is shown in Fig. 3-35 for the Complex shell for the $90^{\circ}$ incidence case. At this incident angle the source should excite only waves which travel circumferencially around the shell. The $0.6 \mathrm{msec}$ periodic peaks are due to each circumnavigation of a pair of compressional waves one in each direction. No axial waves would be present at this incidence angle for an infinite cylindrical shell. However, there are waves visible traveling axially down the length of the shell. 
These waves show that either the endcaps or the ring stiffeners are generating waves with axial components. This will be shown explicitly in Section 3.8 by the Radon Transform.

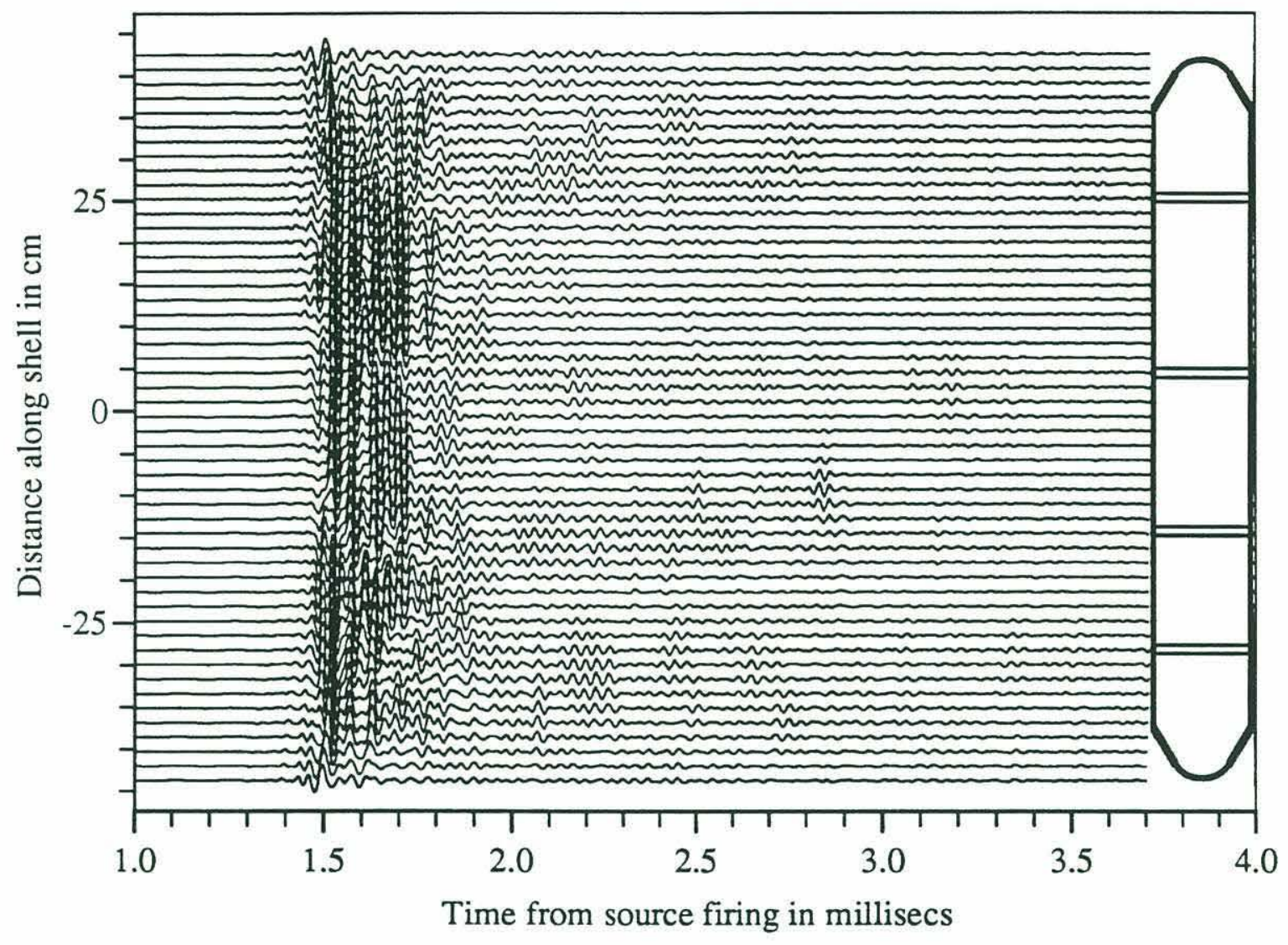

Figure 3-35: $60^{\circ}$ of array around shell beam, geometrically scattered return removed, deconvolved by the source pulse, Hamming tapered and focused onto shell. Complex shell data at $90^{\circ}$ incidence.

\subsection{Energy}

The processed data provides the time response of samples of the shell surface which can radiate to the far field. The focused shell data has both time and spatial extent. It is therefore possible to integrate energy over either of these axes and plot this integral with respect to the other. The spatial integral was approximated as the finite. discrete sum over the 51 traces of processed data, such as that in Fig. 3-26, 
and is defined by:

$$
E_{x}(t)=\int_{0}^{L} y^{2}(x, t) d x \approx \sum_{i=0}^{50} y_{i}^{2}(t)
$$

Fig. 3-36 shows the energy integrated over the shell at each time sample for the $0^{\circ}$ incidence case. The bold line shows the Empty shell response, the plain shows the Ribbed shell response and the dotted line shows the Complex shell. These curves were smoothed by 50 point moving average windows to show trends. The Empty shell curve shows two peaks at $1.25 \mathrm{msec}$ and another $200 \mu \mathrm{sec}$ later due to the compressional waves on the shell. The energy due to these waves seems to decay at about $30 \mathrm{~dB} / \mathrm{msec}$. Later in time, at $2.25 \mathrm{msec}$, the flexural waves begin to add significantly to the radiated energy as they couple into compressional waves. The Ribbed and Complex shell energy plots have two early peaks as well, but they decay at a much lower rate, about $10 \mathrm{~dB} / \mathrm{msec}$, and they do not have the late flexural arrival. It is hypothesized that this is a result of the flexural and compressional waves coupling at each stiffener and endcap. Thus, energy is being released from the flexural wavetype more uniformly in time by the dense stiffener distribution and contributing strongly to the radiated energy of the Ribbed and Complex shells. The energy curve for the Ribbed shell drops off earlier than the other two. This is simply due to a shorter time window used during acquisition.

Similarly, the time integral was approximated as the finite, discrete sum over 1501 time samples, and is defined by:

$$
E_{t}(x)=\int_{0}^{\infty} y^{2}(x, t) d t \approx \sum_{n=0}^{1500} y_{x}^{2}\left(t_{n}\right)
$$

Fig. 3-37 shows the energy integrated over time for each spatial sample of the shell at $0^{\circ}$ incidence for all three shells. Notice that the energy is distributed fairly uniformly over the Empty shell, except for peaks in the endcap region. Work by Guo [10] has shown that compressional waves radiate more strongly as they propagate down the conical endcap section due to the necking down of the shell radius. However, some work by Conti and Dyer [28] suggests that the cone couples to the spherical section before a critical radius for radiation is reached. It is therefore thought that the energy 
peaks at $\pm 40 \mathrm{~cm}$ is due to the compressional waves recoupling into the water from the spherical portion of the endcap.

In contrast, the energy in the Ribbed and Complex shells gradually decreases along the length of the shell. Energy in each subsequent bay drops by about $5 d B$. The return at the bow endcap is about $3 d B$ higher than for the Empty shell, since the first stiffener reflects most of the first wave energy, essentially doubling the energy. There are also noticeable peaks in the return corresponding to the locations of the stiffeners. It was originally thought that these were entirely due to diffraction at those discontinuities and raises a discrepancy in the next section on resolution. The actual source of these peaks remained obscured until the forward modeling of the Ribbed shell was completed. The wave in the water passing down the shell also seems to introduce energy at the stiffeners. This can be seen as peaks of energy along a line connecting points $(1.25 \mathrm{msec},-43 \mathrm{~cm})$ and $(1.83 \mathrm{msec}, 43 \mathrm{~cm})$ in Figs. $3-28$ and $3-27$, which corresponds to the moveout of the water wave. Notice that there is a $3 d B$ difference between the the energy at the stern of the Ribbed and Complex shells. This is evidence that some of the energy has been transfered into the wave-bearing internals rather than to radiation

Fig. 3-37 also brings up an important issue: array resolution. As indicated above, it was hypothesized that the peaks in the curves for the Ribbed and Complex shells were due to diffraction from the rib stiffeners. The stiffeners are welded to the shell essentially on a circle on the shell, a point in this 1D plot. However, the finite length array is not able to locate the ring stiffeners to infinite precision. The width of the energy peak near the stiffener location is approximately $8 \mathrm{~cm}$. This was assumed to indicate the array resolution limit. Two waves spaced more closely than this limit will be seen as a single wave, rather than two. The resolution limit affects our ability to determine certain shell properties. For example, to determine the reflection coefficients at a stiffener, it is necessary to estimate the in-going and out-going waves at the stiffener. The reflection coefficient is estimated by dividing the frequency responses of these two waves. Fig. 3-26 shows that we might be able to estimate a wave and its reflection at the endcap of the Empty shell, at $(1.40 \mathrm{msec}, 35 \mathrm{~cm})$ for 
example, but the stiffeners on the Ribbed and Complex shells create waves which overlap spatially within the time resolution. Visually, in the focused data of Figs. 327 and $3-28$, it is not possible to pick out a single wave and its reflection at a stiffener or endcap for the Ribbed or Complex shell as it is for the Empty shell. The waves are buried by the beampattern of the array.

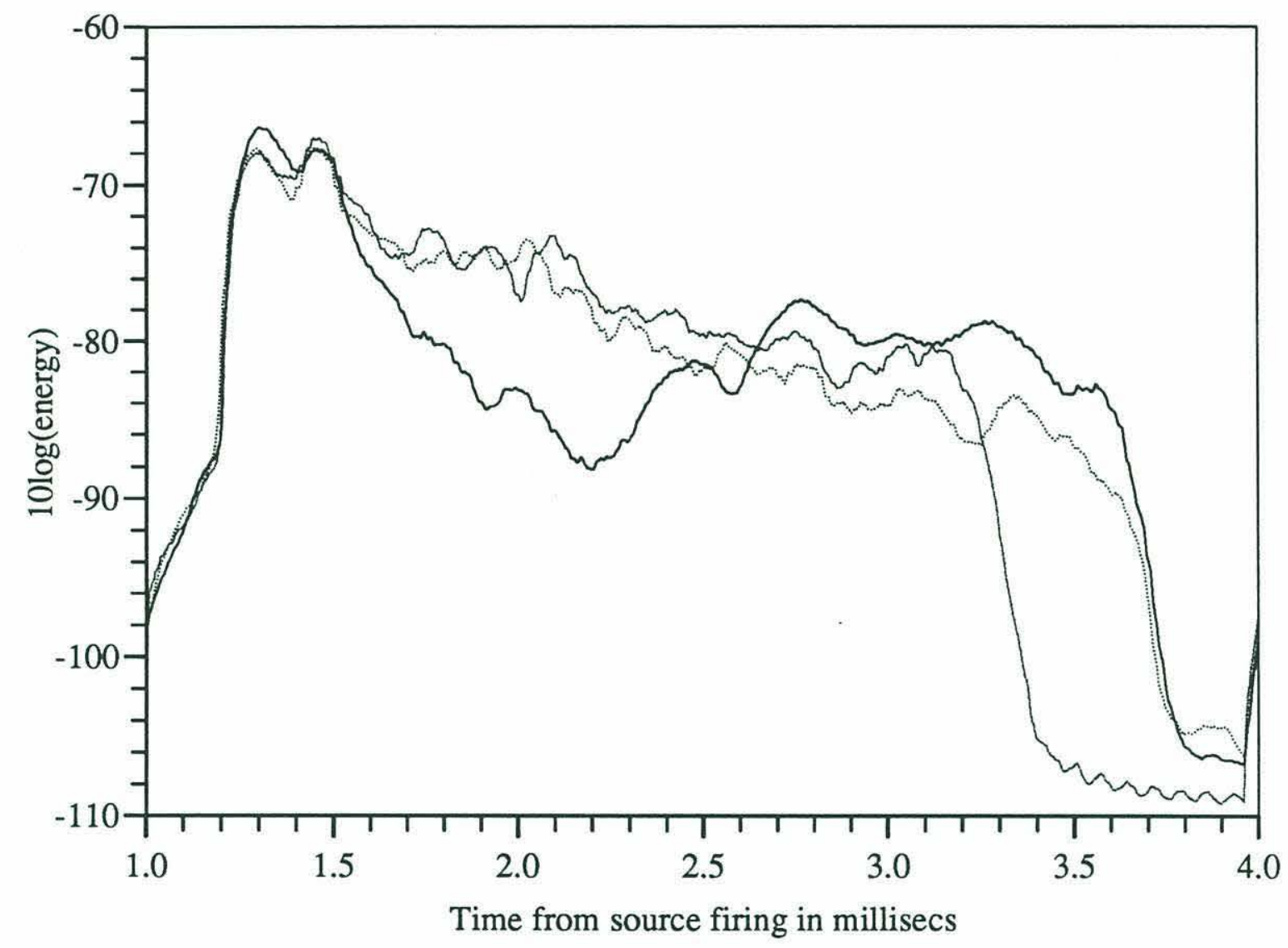

Figure 3-36: Response of shell surface, energy integrated over space. Empty (bold), Ribbed (plain) and Complex (dotted) shells for $0^{\circ}$ incidence case.

Figs. 3-38 and 3-39 shows the energy integrated over the shell at each space and time sample respectively for the case of $75^{\circ}$ incidence. The bold line shows the Empty shell response, the plain line shows the Ribbed shell response and the dotted line shows the Complex shell. The time decay of energy of the shells is very similar to the $0^{\circ}$ incidence case. The Empty shell shows a $20 d B / m s e c$ decay early then $5 d B$ late in time as flexural waves become important. The Ribbed and Complex shells show a $8 d B$ decay throughout time. There is great similarity between the spatial energy 


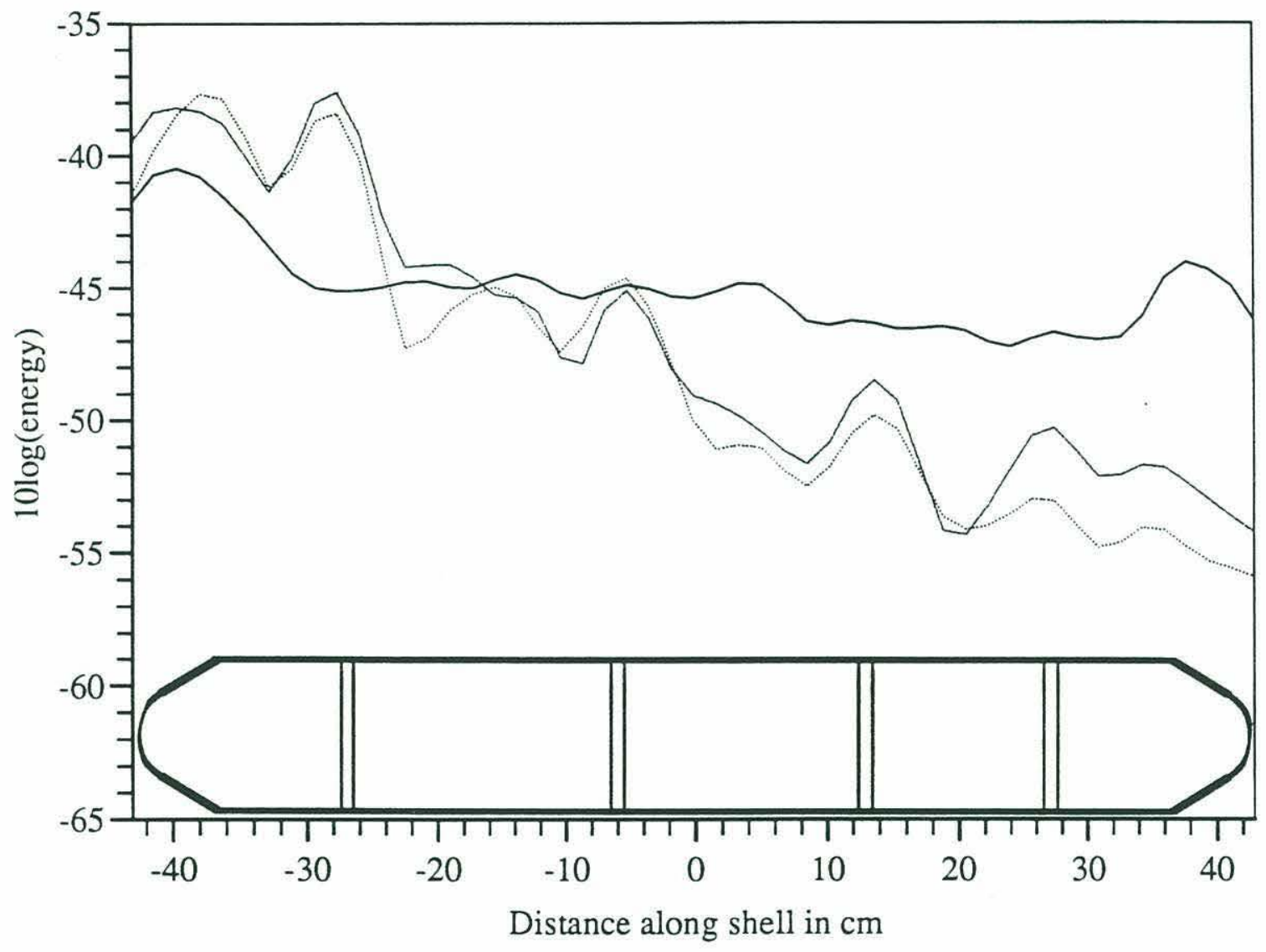

Figure 3-37: Response of shell surface, energy integrated over time. Empty (bold), Ribbed (plain) and Complex (dotted) shells for $0^{\circ}$ incidence case. 
distributions of the Empty and Ribbed shells at this incidence angle. The energy is distributed along the shell fairly uniformly by the source pulse, so compartmentalizing by the ribs does not affect the spatial decay as it did in the $0^{\circ}$ incidence case. Notice, however, that the energy of the Complex shell both in space and in time is uniformly $3 d B$ lower than that in the Ribbed shell. This shows that half the energy of the pulse has gone into processes associated with the internals rather than into radiation.

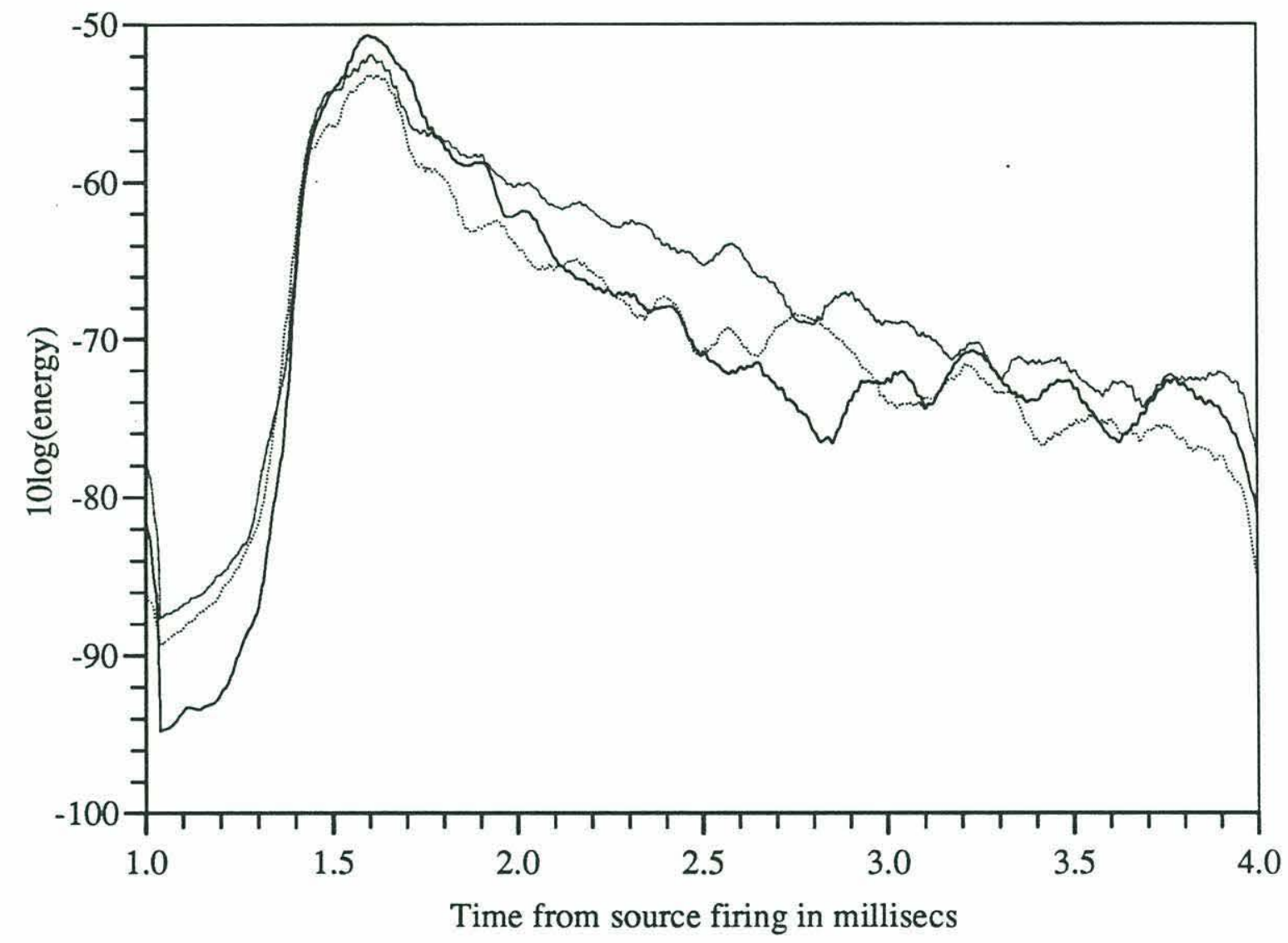

Figure 3-38: Response of shell surface, energy integrated over space. Empty (bold), Ribbed (plain) and Complex (dotted) shells for $75^{\circ}$ incidence case.

Finally, Figs. 3-40 and 3-41 show a comparison of the energies for the Complex shell at $66^{\circ}$ and $75^{\circ}$ incidence over space and time respectively. The energy levels between these two shells are very similar. The difference between these two angles is the inclusion of source excited compressional waves in the $75^{\circ}$ case. It would seem that shear waves are the dominant wavetype in this incident angle regime since compressional waves decay quickly as evidenced by the $2 d B$ higher energy early in 


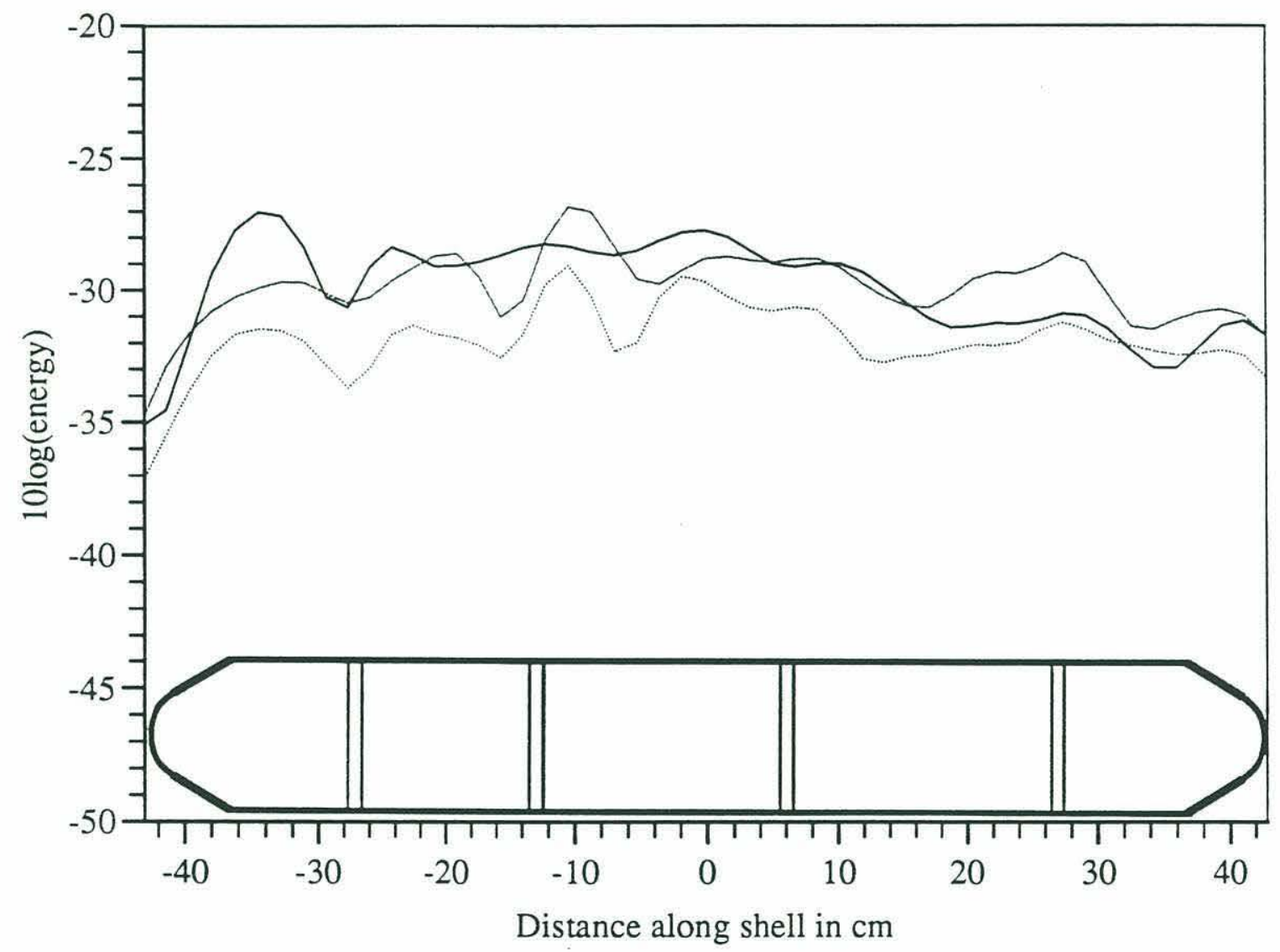

Figure 3-39: Response of shell surface, energy integrated over time. Empty (bold), Ribbed (plain) and Complex (dotted) shells for $75^{\circ}$ incidence case. 
time for the $75^{\circ}$ incidence case as seen in Fig. 3-40.

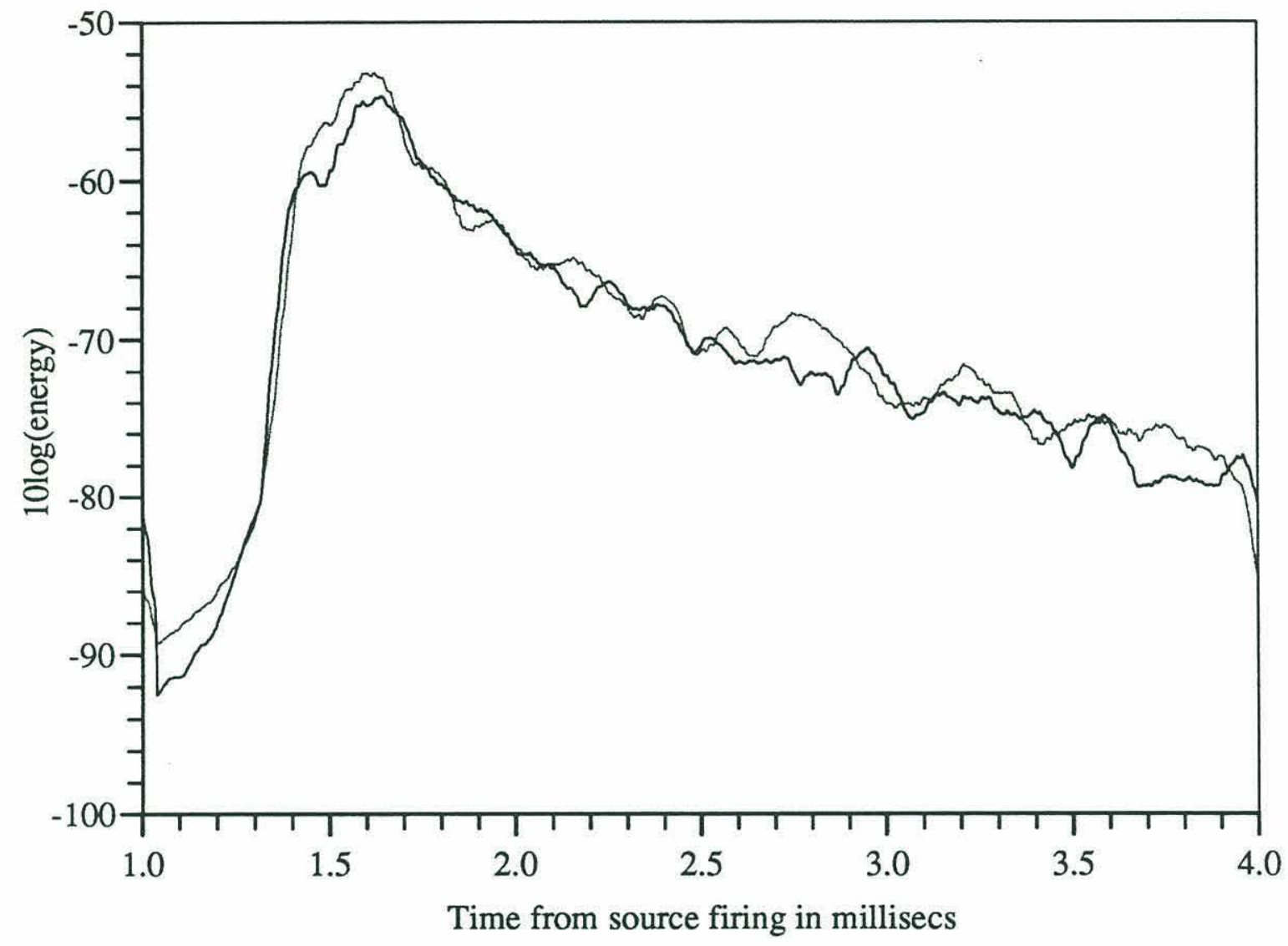

Figure 3-40: Response of shell surface, energy integrated over space. Complex shell at $66^{\circ}$ incidence (bold) and Complex shell at $75^{\circ}$ incidence (plain).

\subsection{Resolution of Array Processing}

This section attempts to quantify the array processing resolution issue. The spatial resolvability of waves traveling on the shell is a function both of the experimental geometry and the processing in the direct analysis leg of the Hybrid Processing Structure. First, an experimental estimate of the resolution is made using the energy measurements of Section 3.6 for an optimal MLM beamformer, then an analytic theoretical limit is computed for this processing via the Cramer-Rao bounds. 


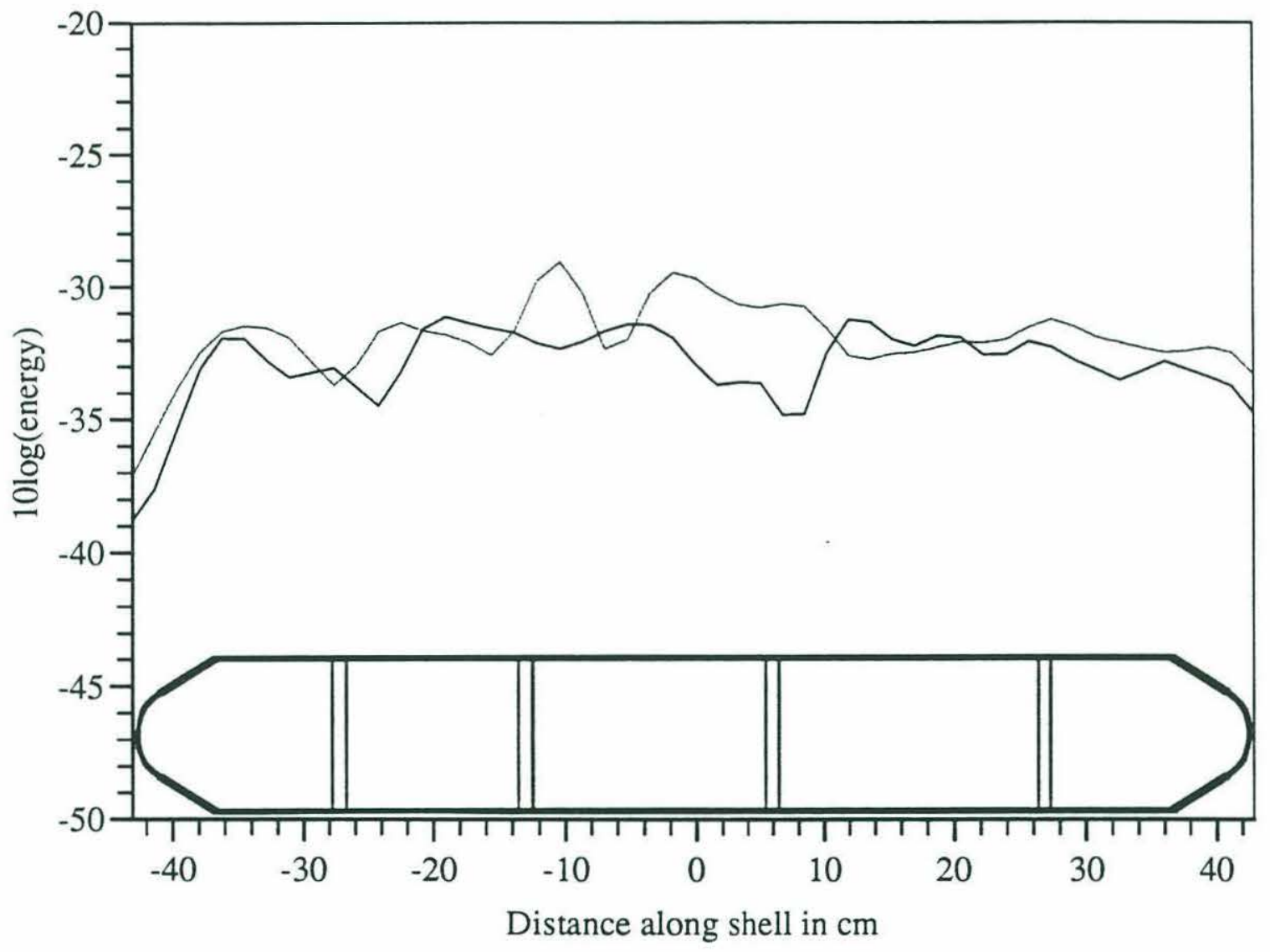

Figure 3-41: Response of shell surface, energy integrated over time. Complex shell at $66^{\circ}$ incidence (bold) and Complex shell at $75^{\circ}$ incidence (plain). 


\subsubsection{Results of MLM Beamforming}

The MLM beamformer is an adaptive extension of the conventional focusing as shown in Section 2.4. This beamformer was applied to data in the same way as the conventional focusing, except the Hamming taper was not applied to the data before beamforming. The geometrically scattered return removal process and the source deconvolution were both still employed.

The results of the MLM beamformer are shown in Fig. 3-42 for the Ribbed shell data for the $0^{\circ}$ incidence case. Notice that this plot is very similar to Fig. 3-28.

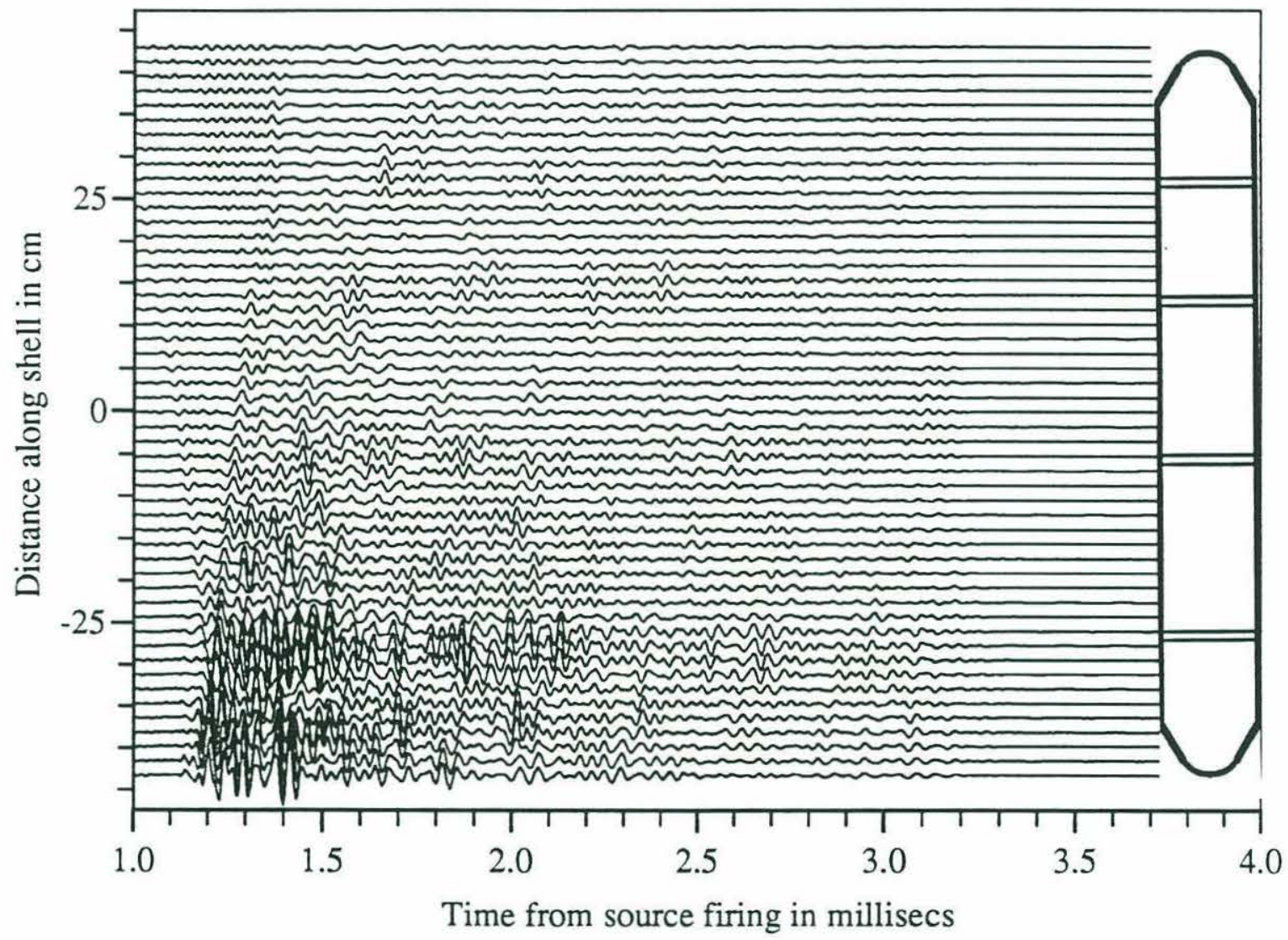

Figure 3-42: $60^{\circ}$ of array around shell beam, geometrically scattered return removed, deconvolved by the source pulse, and MLM focused onto shell. Ribbed shell data at $0^{\circ}$ incidence.

Fig. 3-43 shows the spatial distribution of energy on the shell for the Ribbed shell at $0^{\circ}$ incidence for conventional focusing as a bold line and for the MLM as the plain line. 


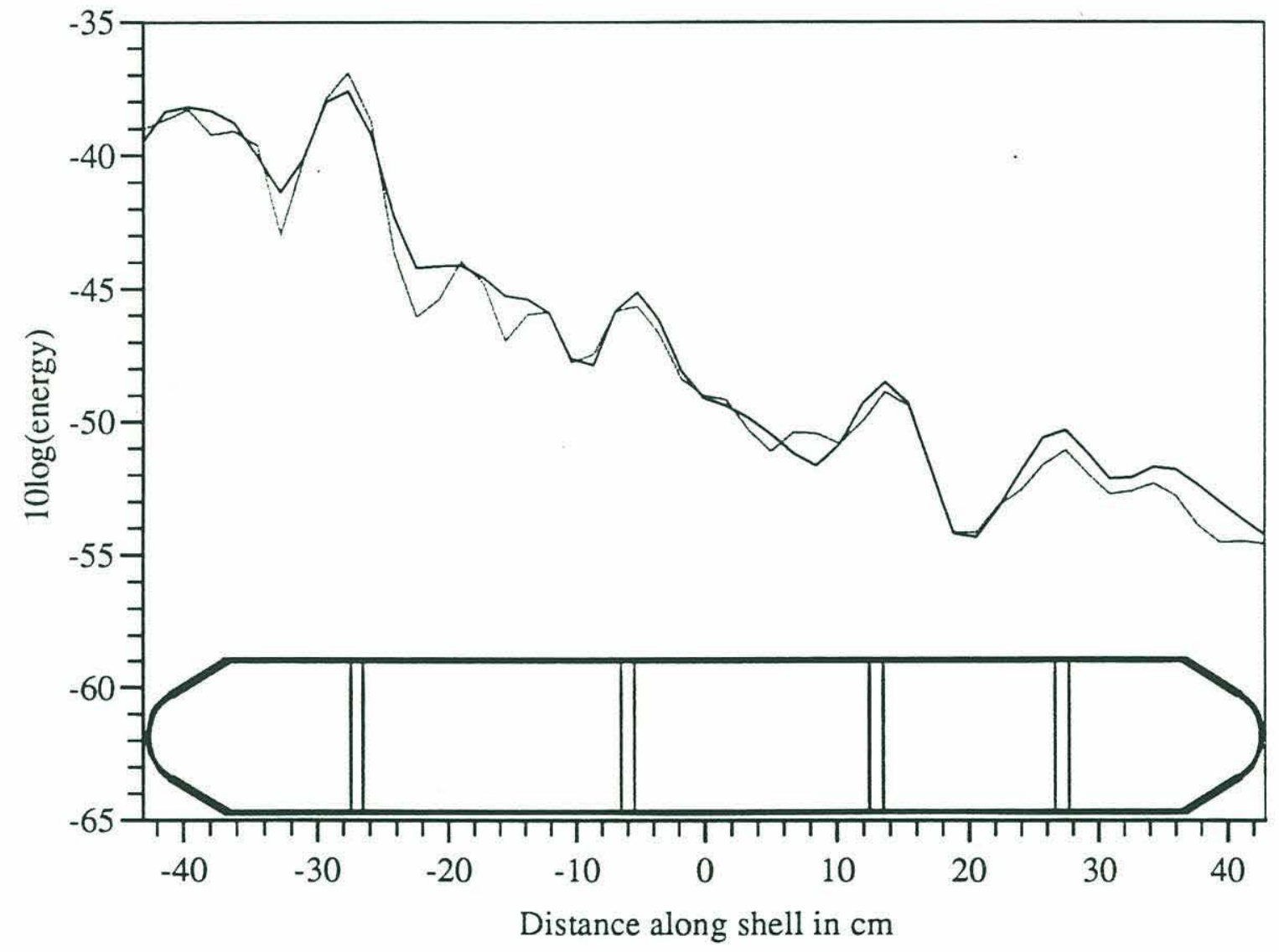

Figure 3-43: Response of shell surface, energy integrated over time. Ribbed shell for $0^{\circ}$ incidence case. conventional (bold) and MLM focusing (plain). 
The spatial energy distribution for the Ribbed shell shows that the MLM is not much better than conventional focusing in terms of the sharpness of these peaks. This could be true for several reasons: (1) The conventional focusing may have reached the theoretical limit of resolution along with the MLM. (2) The MLM has been degraded to the performance of the conventional focusing by the focusing model selected. (3) These peaks are not a good indicator of resolution. This can be answered partially by calculating the theoretical limit for this model.

\subsubsection{Cramer-Rao Bounds}

From probability theory, the Cramer-Rao bound (CRB) is the theoretical limit on the variance of a random variable, $a_{i}$ from its true value, $a_{T i}$. This bound is expressed in terms of the Fisher Information matrix, $\mathbf{J}$.

$$
E\left[\left(\hat{a}_{i}-a_{T i}\right)^{2}\right] \geq\left[\mathbf{J}^{-1}\right]_{i i}
$$

where $E$ is expectation and

$$
\mathbf{J}_{i j}=-E\left(\frac{\partial^{2}}{\partial a_{i} \partial a_{j}} \ln P_{\hat{r} \mid a}(\mathbf{R} \mid \mathbf{a})\right)
$$

where $P$ is the conditional probability density of the field given parameters, $a_{i}$, and the observables, $R$.

The resolution limit of an optimal beamformer can be formulated in these terms. Specifically, if the observables are the Fourier components of the signal and are Gaussian in nature, Baggeroer et al. [29] have show that the Fisher Information Matrix is a function of the input signal covariance:

$$
\mathbf{J}_{i j}=-T R\left(\mathbf{K}^{-1}\left(\mathbf{a}_{T}\right) \frac{\partial \mathbf{K}\left(\mathbf{a}_{T}\right)}{\partial a_{T i}} \mathbf{K}^{-1}\left(\mathbf{a}_{T}\right) \frac{\partial \mathbf{K}\left(\mathbf{a}_{T}\right)}{\partial a_{T j}}\right)
$$

The model for the covariance matrix used in this analysis will be identical to the model used in the focusing problem without amplitude spreading in order to understand its 
theoretical limits. In this case, the covariance matrix is given by:

$$
\mathbf{K}(\mathbf{a})=\sigma_{w}^{2} \mathbf{I}+\sigma_{c}^{2} \Phi_{c} \Phi_{c}^{\dagger}+\sigma_{\tau}^{2} \Phi\left(a_{T}\right) \Phi^{\dagger}\left(a_{T}\right)
$$

The first term in this matrix is the contribution due to uncorrelated sensor noise. The second term is the colored noise term due to 51 sources on the shell surface.

$$
\left(\Phi_{c}\right)_{n}=\sum_{i=0}^{51} e^{-(j 2 \pi / \lambda) d_{n, i}}
$$

where $d_{n, i}$ is the distance from the $i$ th source to the $n$th receiver. The last term is the correlation of the field due to a source of interest located on the shell surface at the horizontal offset $a_{T}$. The Cramer-Rao bound of this parameter will give the theoretical minimum variance of this estimate, i.e. the minimum theoretical resolution of the process. The source field is:

$$
\left(\Phi\left(a_{T}\right)\right)_{n}=e^{-(j 2 \pi / \lambda) d_{n}\left(a_{T}\right)}
$$

where $d_{n}\left(a_{T}\right)$ is the distance from the source at $a_{T}$ to the $n$th receiver.

For this experiment the SNR is excellent, $50 \mathrm{~dB}$, for signal versus the white noise component. The signal to colored noise source, however, is about $-35 d B$ as all the virtual sources on the shell have the same strength. For a source in the middle of the shell, $a_{T}=0$, the CRB over the frequency range of interest is shown in Fig. 3-44.

The figure shows that resolutions of under $0.05 \mathrm{~cm}$ should be possible with this model in the frequency band of interest. This is two orders of magnitude smaller than the $8 \mathrm{~cm}$ shown in Fig. 3-42. It was originally thought that the MLM had been degraded two orders of magnitude in resolution by model mismatch. Model and source mismatch essentially leads to main lobe nulling of the source signal and is the main reason why the MLM beamformer does not achieve the theoretical limit in practice. The sensitivity to model mismatch has been studied in depth in [30, 31, 32].

Another hypothesis was that the CRB for this problem was computed from too simple a model. The theoretical bounds for the actual problem could be found by 


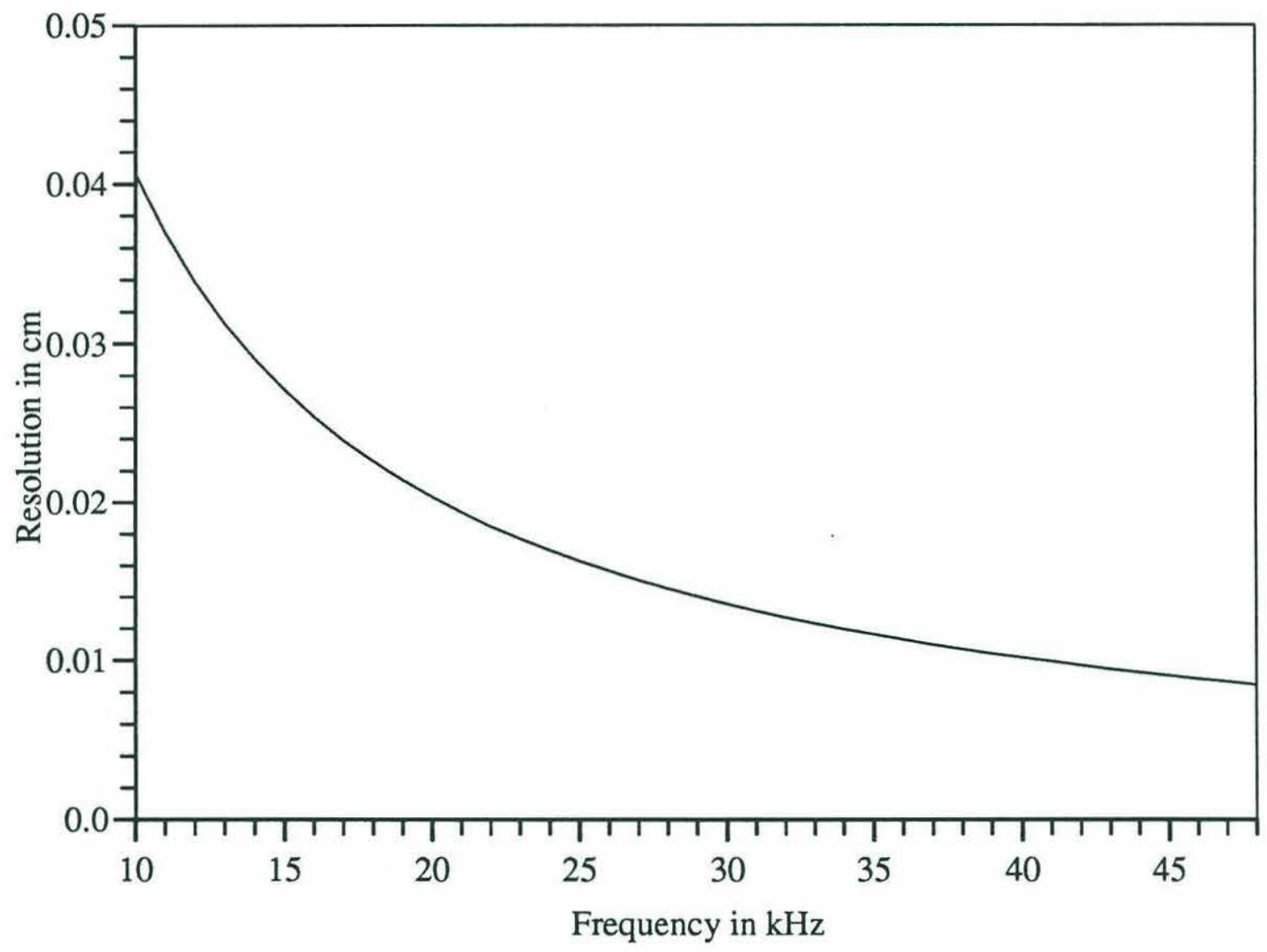

Figure 3-44: Cramer-Rao bound estimate of resolution of source in presence of sensor and line array noise. 
including the correct colored noise covariance matrix into the CRB calculation. This true model is found by solving the problem of how the shell is actually radiating. This is equivalent to matching the model developed in the forward modeling leg of the Hybrid Processing Structure back to the original data, i.e. solving the original problem by forward modeling. Thus, the actual CRB of this problem is probably not computable.

Neither of these hypotheses turns out to be correct. It will be shown through forward modeling in Section 5.4 that both the conventional and MLM beamformers are probably achieving close to the theoretical spatial resolution shown in 3-44 but that the peaks in the energy curves are not a good indicator of that resolution. This will prove the value of the Hybrid Processing Structure.

\subsection{Radon Transform Processing}

The Radon transform will now be used to estimate the compressional wave reflection coefficient at the endcap in the Empty shell for the $0^{\circ}$ incidence case. This use of the Radon transform is similar to work by Fricke and Baggeroer [33] where the Radon Transform was used to isolate overlapping waves of different wavespeeds traveling on

a finite beam. This transform will also be used to show the existence of axial waves on the shell for the $90^{\circ}$ incidence case.

The Radon Transform of the Empty shell focused data from $0^{\circ}$ incidence is shown in Fig. 3-45. Implicit in the transform is a "pivot" line, which is the trace that is not shifted in time over the coherent addition. In this transform, the pivot line is chosen at the discontinuity at the stern endcap, so that the intercept times correspond to time of arrival at the stern endcap for forward traveling waves and time of origination for backward traveling waves. The endcap regions were excluded from this calculation because the diffraction bursts from the spherical portion of the shell do not fit a propagating wave model which is the basis for this transform. A rectangular window with Hamming ends is again used to reduce spatial sidelobes. The middle 37 time traces with a 9 point taper on each end were used for this computation. 


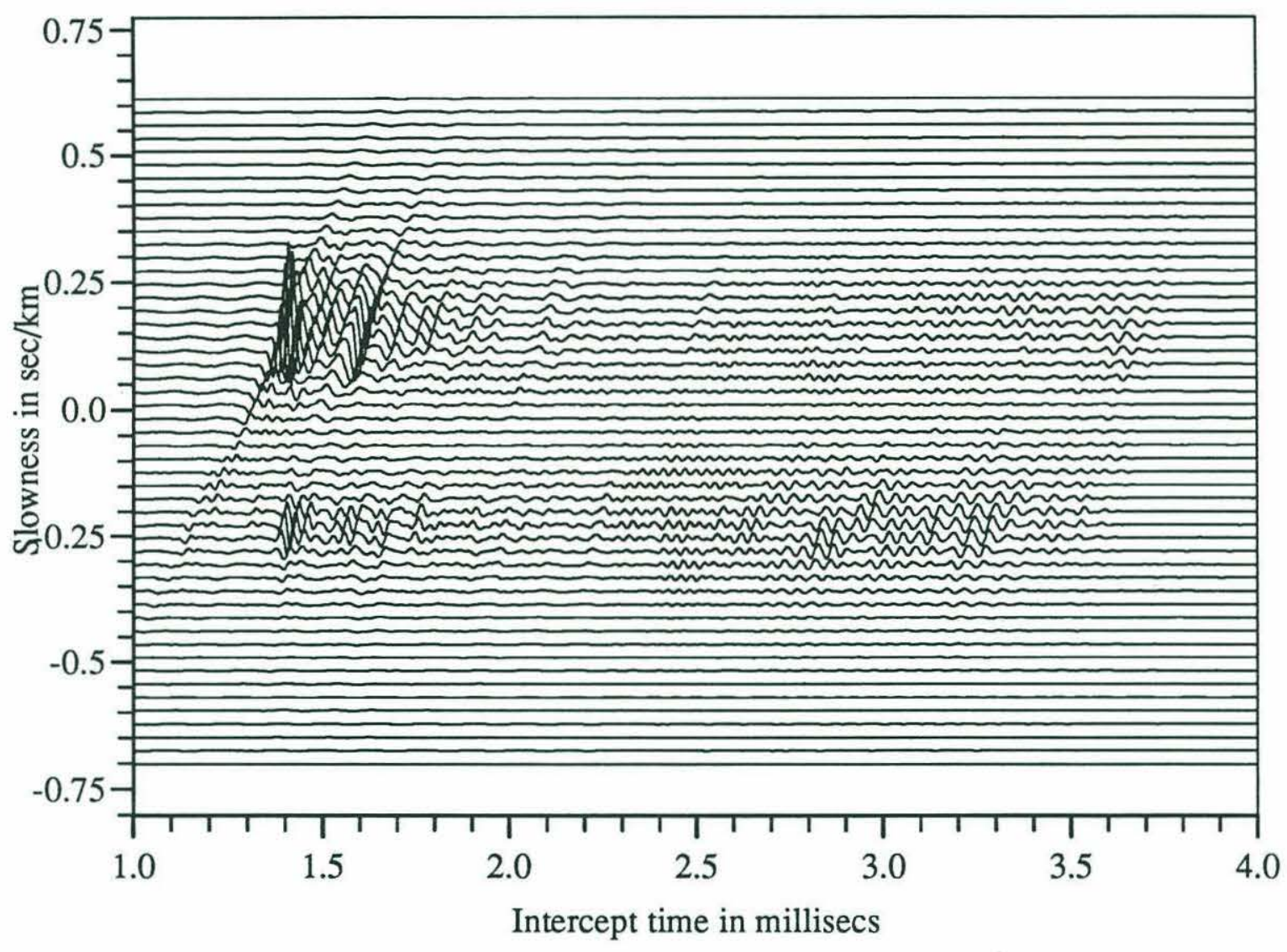

Figure 3-45: Radon transform of waves traveling on the Empty shell using $0^{\circ}$ incidence data. 
There are two peaks of these transforms, located at $p \approx \pm 0.19 \mathrm{sec} / \mathrm{km}$, which is consistent with Corrado's [5] compressional wavespeed estimate, $c_{p}=5270 \mathrm{~m} / \mathrm{sec}$. The width of this peak is controlled by the spatial extent of the data, in this case, the length of the shell. The peak traces are shown isolated in Fig. 3-46 with the forward and backward propagating compressional waves in the upper and lower traces respectively. The first compressional wave occupies times $1.35 \mathrm{msec}$ to $1.45 \mathrm{msec}$ in



Figure 3-46: Radon transform of waves traveling on the Empty shell using $0^{\circ}$ incidence data. Isolated compressional slownesses.

the top trace of Fig. 3-46, and its reflection occupies the same time in the bottom trace. The magnitude of the spectra of the forward and backward waves are shown in Fig. 3-47. Dividing these two complex spectra provides a frequency estimate of the reflection coefficient of compressional waves at the stern endcap. Its magnitude, shown in Fig. 3-48, is fairly constant over frequency and has an average of approximately 0.19 . 


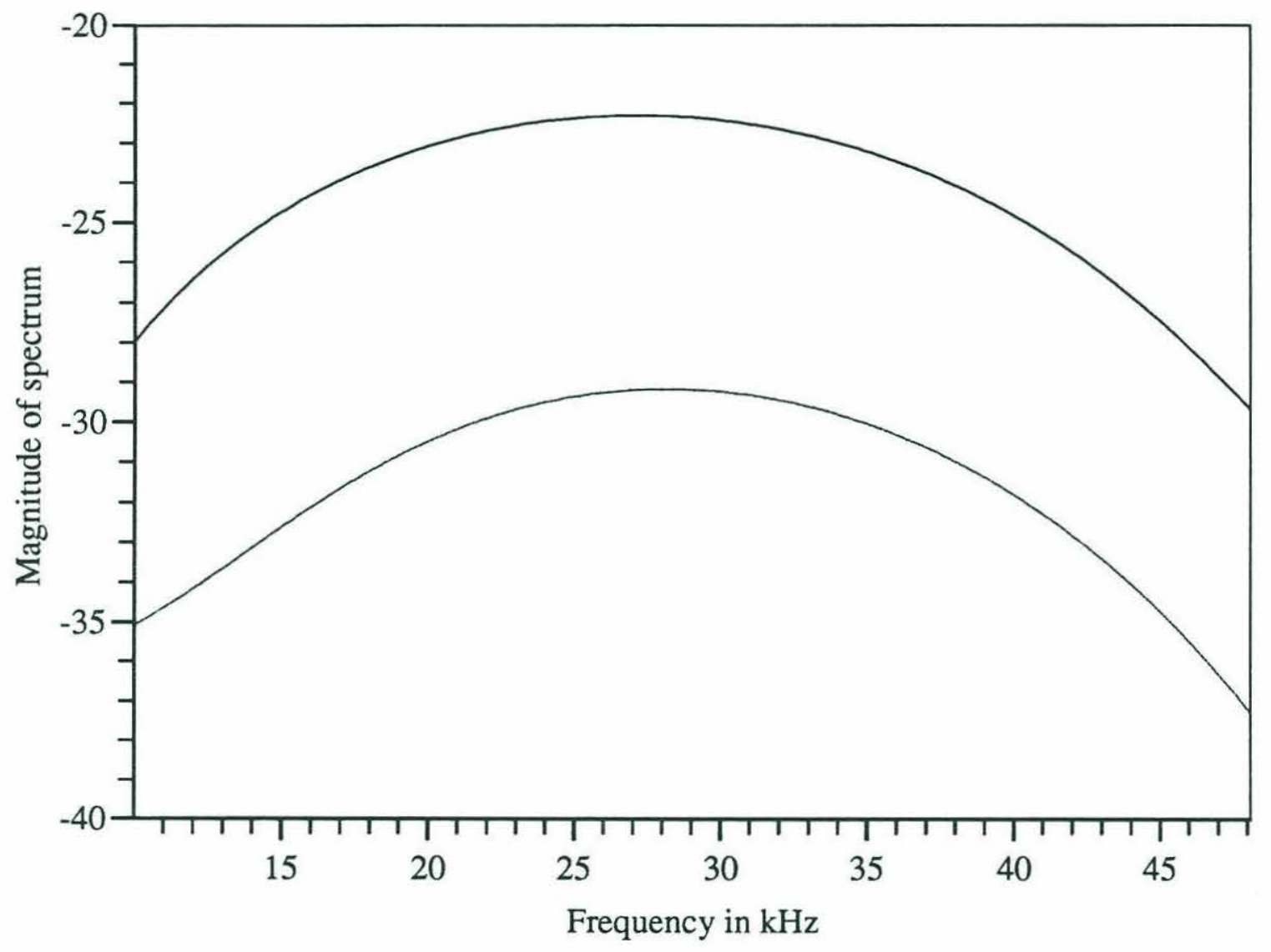

Figure 3-47: Magnitude of spectra of first forward and backward compressional waves. 


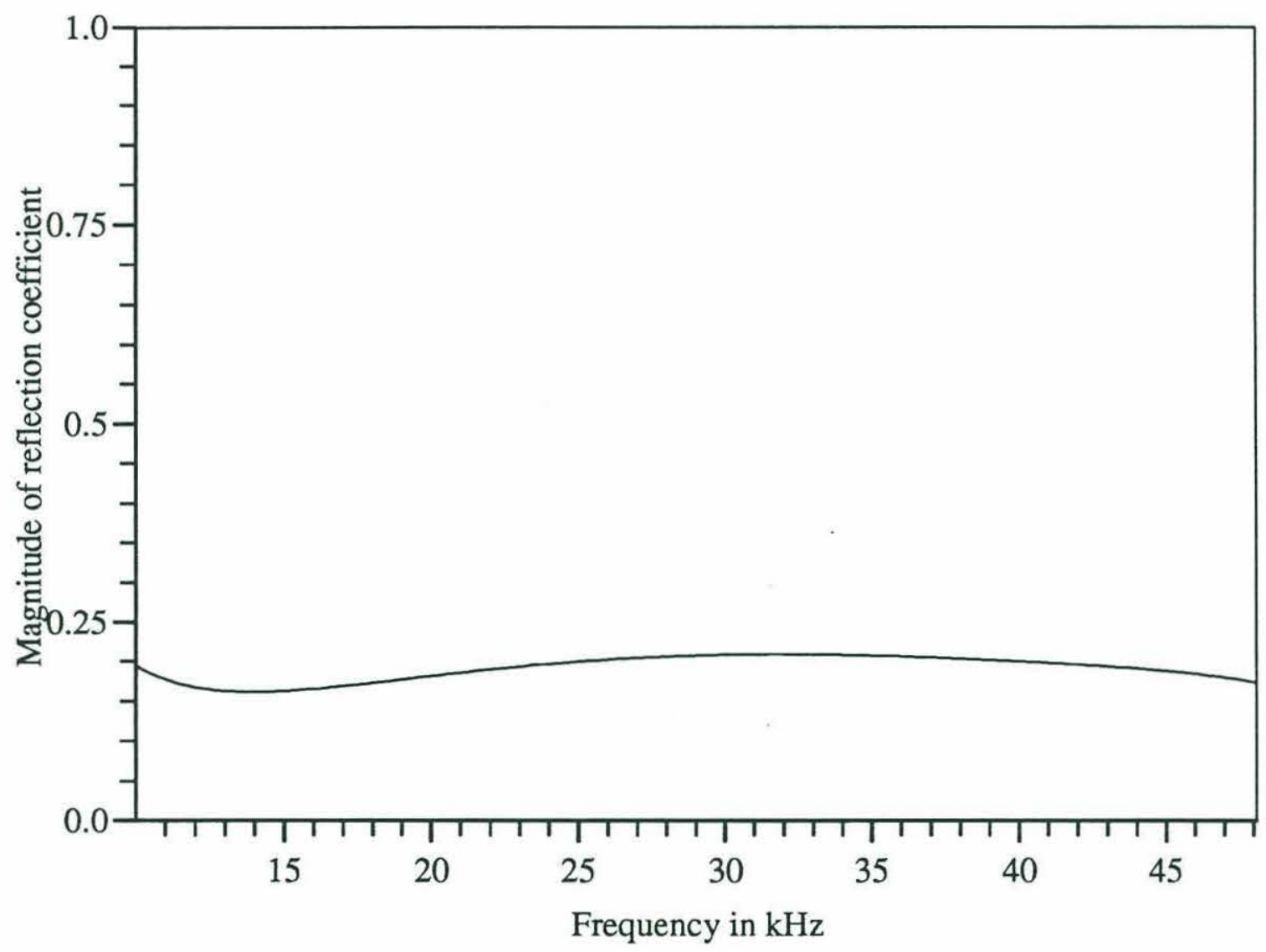

Figure 3-48: Estimate of magnitude of compressional Reflection coefficient at stern endcap of Empty shell from $0^{\circ}$ incidence data. 
Since this direct analysis technique is useful for the Empty shell, it should be useful for estimating the reflection coefficients at the rib stiffeners in the Ribbed or Complex shells. The Radon Transform of the Ribbed shell data from $0^{\circ}$ incidence is shown in Fig. 3-49. The pivot line is selected at the stern endcap once again. In this case, the transform is not useful, because there are multiple stiffeners present, each of which represents a potential pivot location. Notice that the center of the transform space has been filled in, due to the stiffeners physically aliasing the forward and backward traveling waves. This aliasing could be removed by considering only a single bay between discontinuities as the transform space. This would remove the aliasing, but at the cost of increasing the width of the peaks in the slowness coordinate to an unacceptable degree. This is due to the fact that slowness resolution is tied to the spatial extent of the transformed data. For this reason, estimation of reflection, transmission and coupling coefficients at the ring stiffeners cannot be solved by direct analysis. The desire for additional quantitative measurements of this type were the primary motivation for moving on to forward modeling methods.

Finally, the Radon Transform of the focused Complex shell data for the case of $90^{\circ}$ incidence is shown in Fig. 3-50. This time, the pivot line of the transform is chosen at the center of the shell. Since most of the waves move circumferencially around the shell, they have no moveout over the $x$ coordinate. This fills the area of $p=0 \mathrm{sec} / \mathrm{km}$ slowness. Notice, however, that there is some energy at both the compressional slowness of $p \approx 0.2 \mathrm{sec} / \mathrm{km}$ and shear slowness of $p \approx 0.3 \mathrm{sec} / \mathrm{km}$. This provides proof that the endcaps or ring stiffeners are inducing waves with axial components on the shell. 


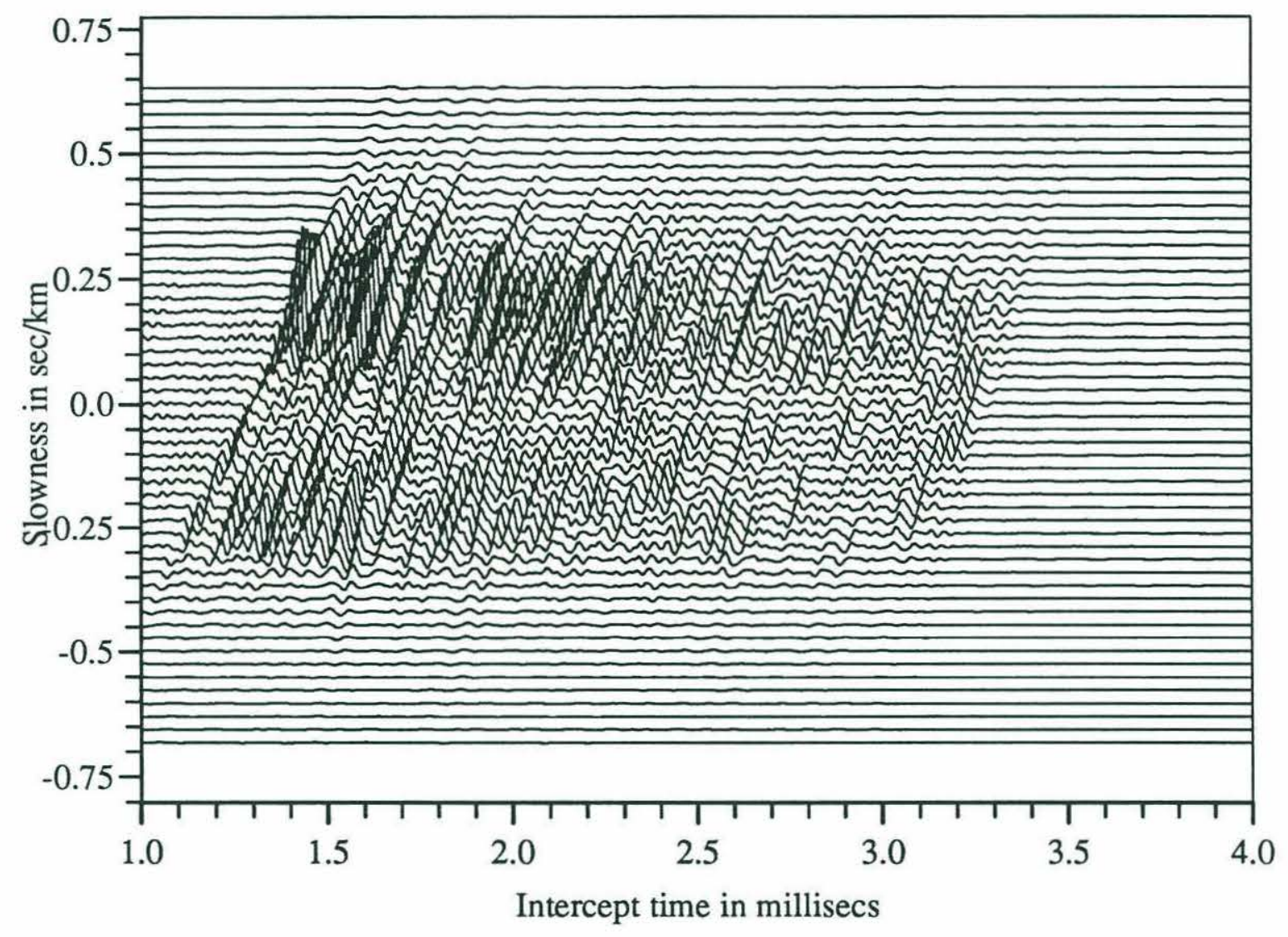

Figure 3-49: Radon transform of waves traveling on the Ribbed shell using $0^{\circ}$ incidence data. 




Figure 3-50: Radon transform of waves traveling on the Complex shell using $90^{\circ}$ incidence data. 


\section{Chapter 4}

\section{Forward Modeling}

\subsection{Motivation}

In Chapter 3, a great deal of processing was performed on the various raw datasets to transform them to a representation which showed the waves traveling on the surface of the three shells. The models used in this process were standard but are an approximation to the physics of the problem. Also, the time and spatial resolution of the direct analysis are limited, even in the case of optimal focusing, by the parameters of the data collection.

The direct analysis leg of the Hybrid Processing Structure would not be necessary if measurements were available at the shell surface. Then there would be no resolution degradation of the data due to array lengths and focusing models and no sidelobe artifacts. There is precedent for such fluid-loaded shell measurements at NRL using a different measurement system $[7,8]$, but not at the frequencies of interest and not for incident plane wave scattering. The physical constraints of the measurement equipment used for MIT's experiments prevented measurements close to the shell. Thus, there is no experimental way to get around the limitations of the direct analysis.

These limitations prevent estimation of the physical variables of interest. The waves which are bouncing around on the shell are overlapped in time and space. This overlapping coupled with poor spatial resolution and sidelobes contamination prevents the effective isolation of single waves traversing the shells. Without this 
ability, it is practically impossible to extract decay rates, the dispersion relation for flexural waves or the coupling coefficients between wavetypes. For example, as shown in Section 3.8, the Radon transform can only extract slowness information under the constraint of the available aperture length. For the Empty Shell, the aperture is the entire shell length without the endcaps. Here the Radon Transform was able to extract an estimate of the compressional reflection coefficient at the stern shell/endcap discontinuity. However, the effective apertures for the Ribbed and Complex Shells are the lengths of the bays between the ring stiffeners. These are far too short to allow the Radon transform to extract meaningful slowness estimates.

Finally, even if the resolution were improved, certain datasets are not complete enough for further quantitative analysis. Any incidence angles which induce waves with a circumferencially traveling component cannot be captured fully by this measurement geometry. The collection array has no resolution in the circumferencial direction as it has no extent in the direction perpendicular to the shell axis. Thus, for example, the helical waves of the $75^{\circ}$ incidence case can only be described qualitatively. In fact, the only datasets which can be analyzed further are those datasets which induce symmetric responses on the shell surfaces. This would include the $0^{\circ}$ incidence datasets only. Even these datasets suffer from the fact that at this measurement radius, the properties of the flexural waves traveling on the shells can only be indirectly inferred when they couple into radiating wavetypes.

Since there are limits to the applicability of direct analysis, other techniques must be applied to this dataset. Thus, this chapter describes the forward modeling techniques used to implement the second leg of the Hybrid Processing Structure. Forward modeling has been used successfully in many cases where the data is too complex to understand completely [34, 35]. Simple models described by a reduced number of parameters are often able to match major features of data and provide at least partial understanding of the underlying phenomenology. A block diagram of the forward modeling structure used to match the processed experimental data in this thesis is shown in Fig. 4-1. Notice that this approach is in the form of a loop, it is in fact iterative in nature. This form dictates that the processing time through the loop 


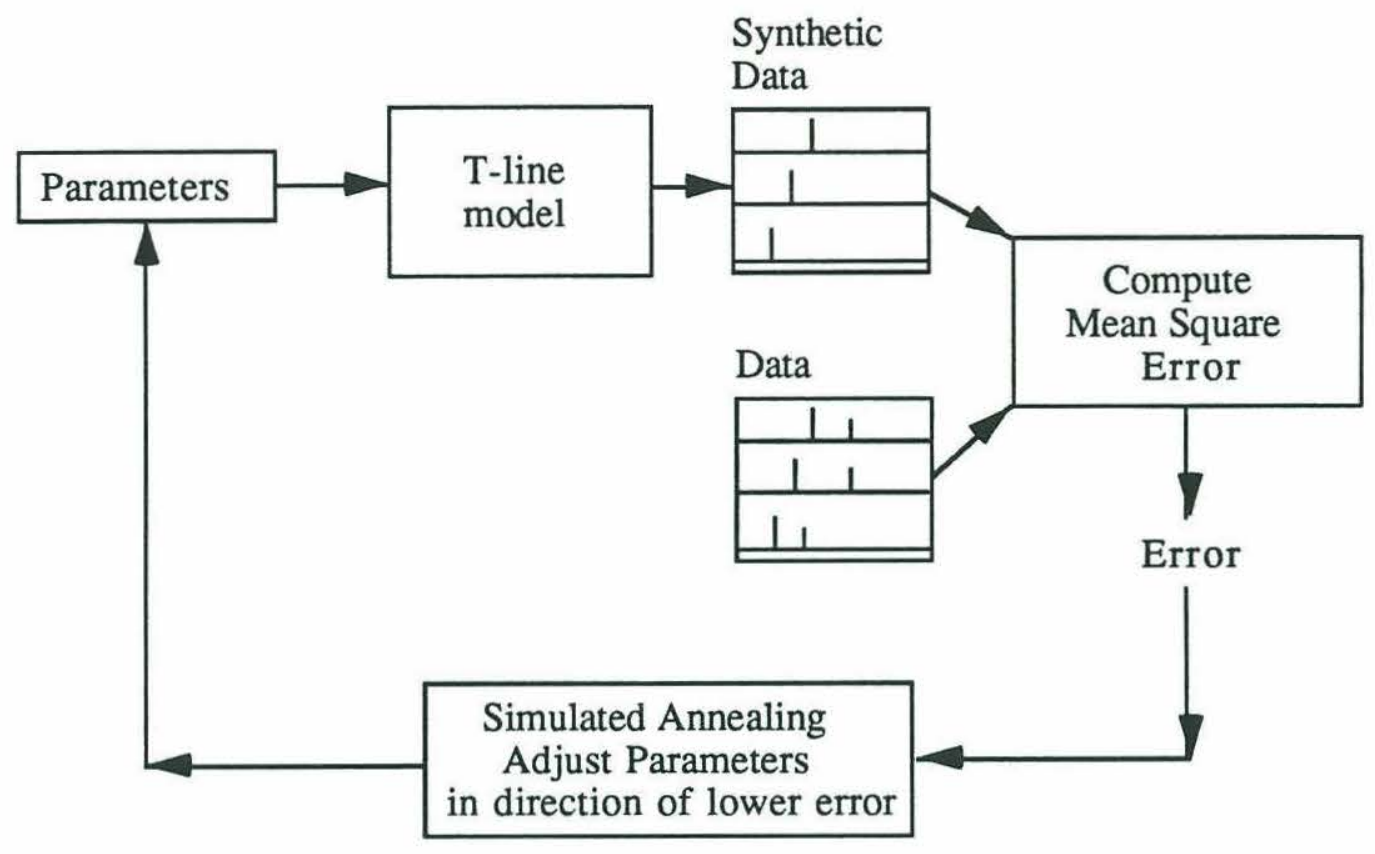

Figure 4-1: Forward modeling structure.

must be short if the number of iterations is expected to be high. The model should be designed so that the input parameters which direct it have some physical significance to the problem at hand. The initial values of these parameters can then be chosen within physically realizable ranges. These values are fed into the model which generates synthetic data of the same dimensions as the data to be matched. Next, an error is generated. In this case, the synthetic data is compared to the real data by computing the total mean square error between the two. This error is fed to a module which adjusts all parameters such that the next error should be lower and begins the loop again. This iteration continues until the error changes by less than some tolerance chosen by the user. At that point, the simulated data represents the best fit to the data for the given model and the parameters represent the optimal parameters for that model. The final parameter values are also a good check on the validity of the model. If the parameters represent physically meaningful variables, their final values should fall within physically meaningful ranges.

This is the same type of structure used in most forward modeling applications. The difference in this method lies in the data to which the model is matched. Rather than trying to match the data collected at the array, the model is used to match 
the processed dataset which represent only the waves traveling on the shells. The result of this simple change is an enormous reduction in the complexity and computational requirements of the model. This reduction in complexity comes at a price, however. The resolution and sidelobe issues discussed above will affect the modeling and degrade its performance. Some of this degradation is partially made up by the effectively "infinite" resolution and accuracy of the modeling approach.

The structure shown in Fig. 4-1 requires the definition of two modules, a model to generate synthetic data and an algorithm to modify the parameters of that model in the direction of lower error. Section 4.2 describes the transmission line model I chose to approximate the behavior of a cylindrical shell at axial incidence. Adjusting the parameters of this model to minimize error puts this into the class of problems known as parameter estimation problems. This can be done analytically for functions that have closed forms or by hand for problems with a small number of parameters whose values are very constrained. However, as the number of parameters grows, such problems are most often solved numerically by computer. Chapter 4.3 describes a probabilistic method, simulated annealing, which shows great promise in being able to solve such problems with computational efficiency.

\subsection{Transmission Line Model}

\subsubsection{Motivation}

These cylindrical shells could be modeled in many ways. In general, cylindrical geometries are modeled by Finite Difference, Finite Element or Boundary Element approximations to the Donnel thin shell equations [2] or full elastic [11] equations of motion for an infinite shell. These methods can then incorporate models for com-

plicated endcap geometries. Although such methods are excellent for synthesis of data, they are almost useless for analysis for several reasons. First, these methods all involve a large computational burden to model every point on the shell, often requiring months of supercomputer time for a single run. This is acceptable for a single 
data synthesis, but not if the model is to be placed in an iterative loop such as that shown in Fig. 4-1. Second, understanding the response at every point on the shell is overkill for the $0^{\circ}$ incidence datasets where the response is axially symmetric. Finally, these models provide no parameterization of the physics present in the dataset they synthesize. They generally compute a time response at a number of locations which is no better than conducting a physical experiment in terms of understanding. The underlying phenomena can only be understood by then performing some direct analysis on the synthetic dataset.

The problem at hand is an axially excited cylindrical shell. Although the shell can support compressional, flexural and shear wave motions, with this excitation, shear waves cannot be generated. Only symmetric wave motions can occur. This restricts the shell to its 0 th order compressional and flexural modes. That is, for each axial location, the shell behaves identically at each circumferencial point. It radiates symmetrically, so the in-plane field measured by this particular set of experiments represents the motion of the entire shell completely. No helical waves can occur, no other circumferencial motion, such as shear torsional waves can occur. In this case, the shell essentially reduces to a finite "beam" with two wavetypes bouncing back and forth over its length. These waves are governed by certain wavespeeds and decay rates and are reflected, transmitted and coupled into each other at discontinuities along the "beam."

This problem is very similar to the normal mode propagation of electro-magnetic waves on a transmission line. A simple transmission line model for the Empty shell is shown in Fig. 4-2. The shell is broken down into sections. The shell and endcap sections are modeled as electrical transmission lines capable of propagating waves. They are connected by parallel impedances. These impedances are used to represent discontinuities like the endcap/shell connection and the ring stiffeners. The simple electro-dynamic transmission line model has been extended to include other physical propagation properties that will be used to model the focused shell data. These extensions include multiple wavetypes which can propagate at different speeds, dispersion to model the flexural waves in the shell, coupling between wavetypes at 


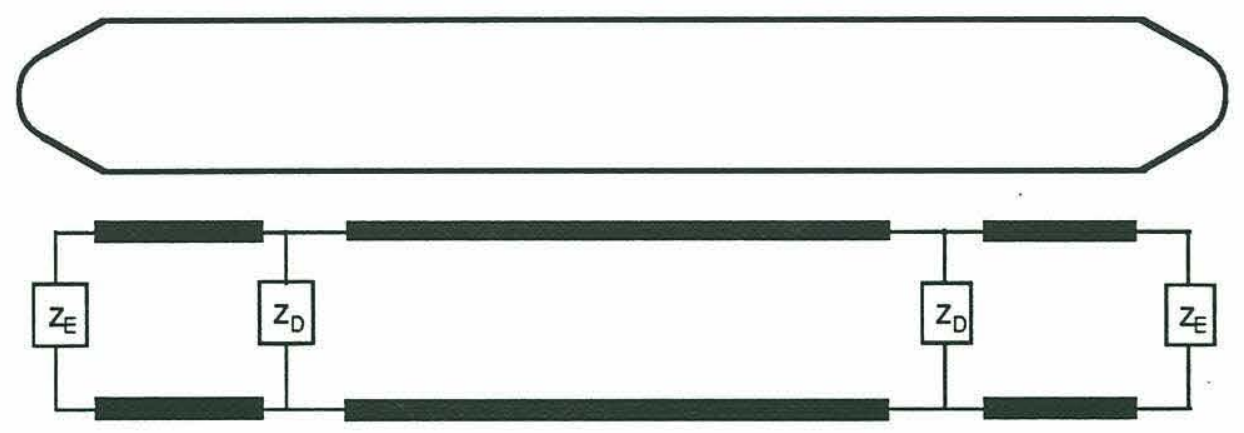

Figure 4-2: Example of a transmission line model for the Empty shell.

discontinuities, decay in time and the option to not show certain wavetypes. This last modification allows propagation of the flexural waves along the transmission line without allowing them "radiate," i.e. be seen in the output. Details of this model are described in the next section.

This model is attractive for several reasons. It has a very intuitive interpretation, so the physics of the shell propagation problem can readily be converted into the model. The data generated by the model can be decomposed into individual propagating waves which can be attributed to easily identifiable sources. Finally and most significantly, this model can be implemented in computationally efficient manner. This last quality makes the model superior to any of the complex models in terms of the analysis problem.

\subsubsection{Model details}

This transmission line model is a very simple implementation of a $1 \mathrm{D}$ wave propagation system. The model has been enhanced beyond the simple electro-dynamic transmission line to emulate the wave propagation properties of the elastic shell. This section describes the inputs and implementation details of this model. An example input file and its description is included in Appendix A. Refer to this for detailed information on how parameters described below are actually specified. In the following description, the term "user" indicates either a human operator or an automatic parameter generation algorithm such as simulated annealing. 
The simulation is defined over a time and spatial extent. Time traces are generated at $N$ positions equally spaced on a transmission line between an $X_{\min }$ and $X_{\max }$ specified by the user. Each time trace is represented by $T$ time samples at intervals $d t=1 / f_{s}$, where $f_{s}$ is the sampling frequency, $T$ and $f_{s}$ are user specified. The user also specifies an upper and lower bandedge, $f_{0}$ and $f_{1}$, for the simulation. All waves on the line are constructed in the frequency domain. The various propagated waves are added together and a single inverse Fourier transform is performed to create time domain data. Only frequencies $f_{0}<f<f_{1}$ are simulated by the model. This reduces the required computation for each wave from $T$ frequency samples to $\left(f_{1}-f_{0}\right) T / f_{s}$ frequency samples, which increases the computational efficiency by roughly a factor of 5 for this particular problem. A floating point value is generated at each of $N \times T$ time-space locations.

The transmission line model operates as a wave object simulation. Each wave is propagated individually according to the rules of its wavetype, the time and spatial extent and the impedances. The user specifies any number of sources, each with its own location, $x_{0}$, starting time, $t_{0}$, and peak amplitude, $a_{0}$. All source pulses in this simulation are Hamming pulses, described in the frequency domain as:

$$
S_{n}(f)= \begin{cases}0.54-0.46 \cos \left(2 \pi \frac{\left(f-f 0_{n}\right)}{f_{b}}\right) & \text { if } f 0_{n}<f<f 1_{n} \\ 0 & \text { otherwise }\end{cases}
$$

where $f_{b}=f 1_{n}-f 0_{n}$ and $f 0_{n}$ and $f 1_{n}$ are the bandedges of the particular source specified by the user. These edges should lie within the overall simulation frequency bandwidth. The source is normalized so the peak amplitude in the time domain is $a_{0}$ and offset so that the left edge of the main beam corresponds to $t_{0}$. This source time pulse is then placed at the spatial location $x_{0}$.

Each source is associated with one of $M$ wavetypes specified by the user. Any number of wavetypes can propagate on the transmission line at once. Coupling between the $M$ wavetypes can not occur on the transmission line during propagation. Coupling can only take place at defined impedance discontinuities which will be described later. 
A wavetype has several different properties. The first is a definition specifying how the wavetype propagates. For each wavetype on the transmission line, a frequency dependence is specified for $c_{g}$, the group delay of the wavetype. The user specifies the group delay value at $P$ frequencies equally spaced over the simulation bandwidth. The model interpolates these points with a $P$ th order polynomial for each required frequency sample. Next the phase delay is calculated from the group delay via integration.

$$
c_{p}(\omega)=\frac{\omega}{\int_{\omega_{0}}^{\omega} \frac{1}{c_{g}(\omega)}+I_{0}}
$$

where $I_{0}$ is the integration constant, which I have simply set to $\omega_{0} / c_{g}\left(\omega_{0}\right)$ where $\omega_{0}=2 \pi f_{0}$. To propagate a wave starting at location $x_{0}$ to location $x$, the frequency response of that wave is multiplied by a complex exponential.

$$
S(\omega, x)=S(\omega) e^{\left(-j \omega t_{0}(\omega, x)\right)}
$$

where $t_{0}(\omega, x)=a b s\left(x_{0}-x\right) / c_{p}(\omega)$. This delays the source pulse to the appropriate time for that spatial location. If $c_{g}$ is constant over the frequency band, the wave packet will retain its Hamming shape and simply be offset by $t_{0}=a b s\left(x_{0}-x\right) / c_{g}$, i.e. the wave will arrive at later time as it travels over space. This is a good model for compressional waves on the shell which are non-dispersive. If $c_{g}$ is a function of frequency, the wave packet will smear out over time as it travels spatially. This is a good model for the flexural waves on the shell which are dispersive.

This transmission line model includes a simple temporal decay as well. The user specifies a decay rate, $\delta$, in $d B / m s e c$. At each spatial location the delayed source is scaled by $10^{-t_{0}\left(\omega_{0}\right) \delta / 20}$. This model provides a linear decay with time.

The user can also specify whether or not the propagating wavetype is added to the output. This allows modeling of flexural waves which travel on the shell but themselves do not radiate. Their influence is seen only when they couple to radiating wavetypes. This ability of the program allows flexural wavetypes to propagate on the shell, interact with impedances and couple to all wavetypes but not themselves added to the output. 
Recognizing that there are parts of the shell such as the endcap sections that propagate wavetypes with different group delays than the main shell section, an allowance was made for this in the transmission line model. The user can specify a spatial range of the transmission line, known as a "slow zone," where each wavetype will propagate via a $c_{g}^{\prime}(\omega)$ curve different from the $c_{g}(\omega)$ originally specified. A slow zone group delay curve is specified by the user for each wavetype in each slow zone. Propagation in these zones is implemented by the same algorithm used for normal propagation described above.

The stiffeners and discontinuities on the shell are modeled by simple parallel impedances on the transmission line. Each impedance has a location and a set of reflection and transmission coefficients which defines the coupling for each incoming $M$ wavetypes to all $M$ outgoing wavetypes. This is an $M \times M$ matrix of reflection coefficients and the same for transmission coefficients. These are specified in amplitude only for this simple model. I felt that phase would be preserved in the endcaps for this problem if distances were preserved. The transmission line segments which represent the endcap are as long as the actual distances along the surface of the cone and sphere. The slope discontinuities should have no phase shifts associated with them, other than -1 which is supported, as there are no material changes here. The ring stiffeners have appreciable mass and here a phase coefficient may indeed be appropriate. However, it is my opinion that the resolution and sidelobe artifacts for the Ribbed and Complex shells will make small phase variations unmeasurable.

Each coefficient can vary over frequency, however. As before the user specifies $P$ amplitude values equally spaced over the frequency bandwidth and the model interpolates using a $P$ th order polynomial. There is also an energy normalization applied so that the energy of the wave going into an impedance at any frequency is equal to the sum of the energy contained in all outgoing waves at that frequency. The interaction of a wave with an impedance is shown in Figs. 4-3 and 4-4. This is similar to some multi-layered media approaches [36].

There are several limits to how long and how many waves propagate on the transmission line. These limits keep the simulation from running for an infinite amount 


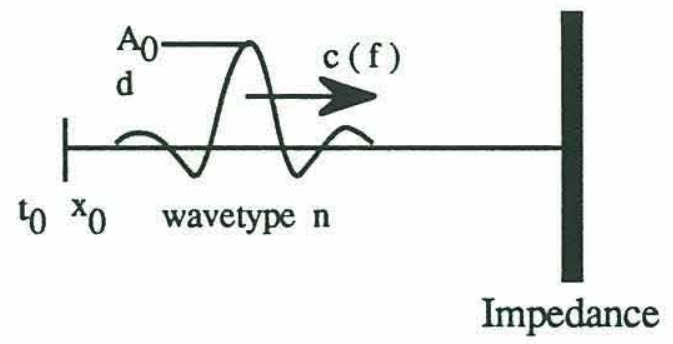

Figure 4-3: Incident wave approaching an impedance.

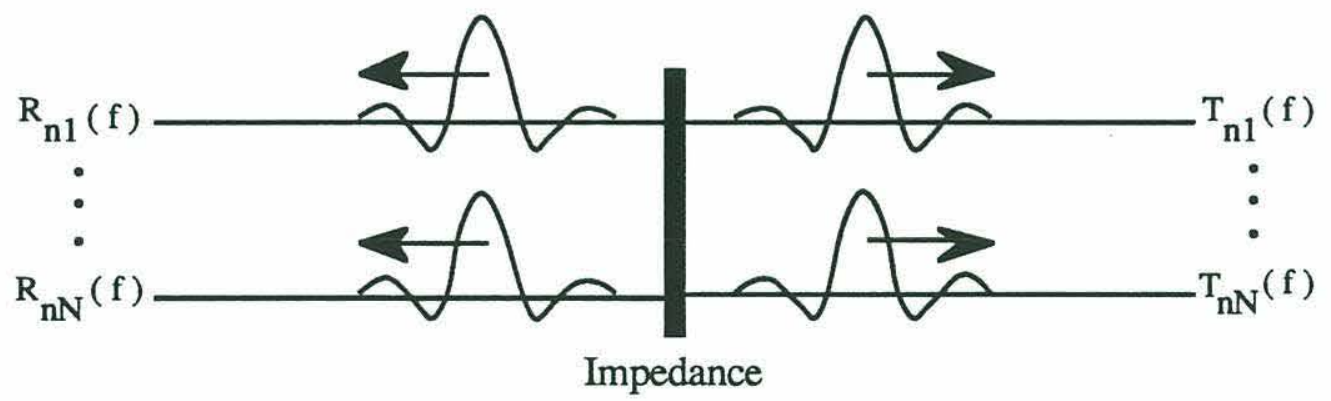

Figure 4-4: Reflected, transmitted and coupled waves leaving an impedance.

of time. Waves continue to propagate in the simulated space until they are terminated by one of three methods. A wave is terminated: (1) When it advances beyond the latest time or space simulated. (2) When its energy has diminished to $X$ of its original value, where $X$ is a model parameter. This allows truncation of very small amplitude waves and has a major effect on computation. (3) When the wave hits a discontinuity. In this last case, reflected, transmitted and coupled waves take the place of the terminated wave. Waves can also fail to be generated at an impedance. A wave is not generated: (1) When more than a user specified number of waves have been generated. (2) When the wave bounces more than a user prescribed number of times. Again these have a major effect on computation.

To clarify the capabilities of this model, several examples which illustrate various features are now presented. All examples are calculated for 51 spatial locations equally spaced over a $1 \mathrm{~m}$ transmission line and 1024 points of time at a sampling rate of $1 \mathrm{kHz}$ for a total of $1.024 \mathrm{sec}$. The simulation is defined over a bandwidth of $50-200 \mathrm{~Hz}$.

Example 1, shown in Fig. 4-5, depicts the propagation of two different wavetypes. Two sources, both of unit amplitude and defined over the entire simulation bandwidth, 
are located at $(0 \mathrm{~m}, 0 \mathrm{sec})$. The first has a wavetype which has a constant group delay over frequency, $c_{g}=4 \mathrm{~m} / \mathrm{sec}$. It also has an $80 \mathrm{~dB} / \mathrm{sec}$ decay rate. This wave arrives at the other end of the transmission line at $0.25 \mathrm{sec}$ and is $20 \mathrm{~dB}$ reduced in amplitude. This non-dispersive, decaying type of wave is a good model for the compressional waves which travel on the shells. The second source has a wavetype which has no decay, however its group delay is a function of frequency. In this case, the group delay varies linearly from $1 \mathrm{~m} / \mathrm{sec}$ at $50 \mathrm{~Hz}$ to $2 \mathrm{~m} / \mathrm{sec}$ at $200 \mathrm{~Hz}$. This dispersion causes the wave to smear out and arrive at the other end of the line between 0.5 and 1.0secs. This dispersive type of wave is a good model for the flexural waves on the shell. Notice that both waves run off the line without artifact, this is a sore point for most Finite Difference models.

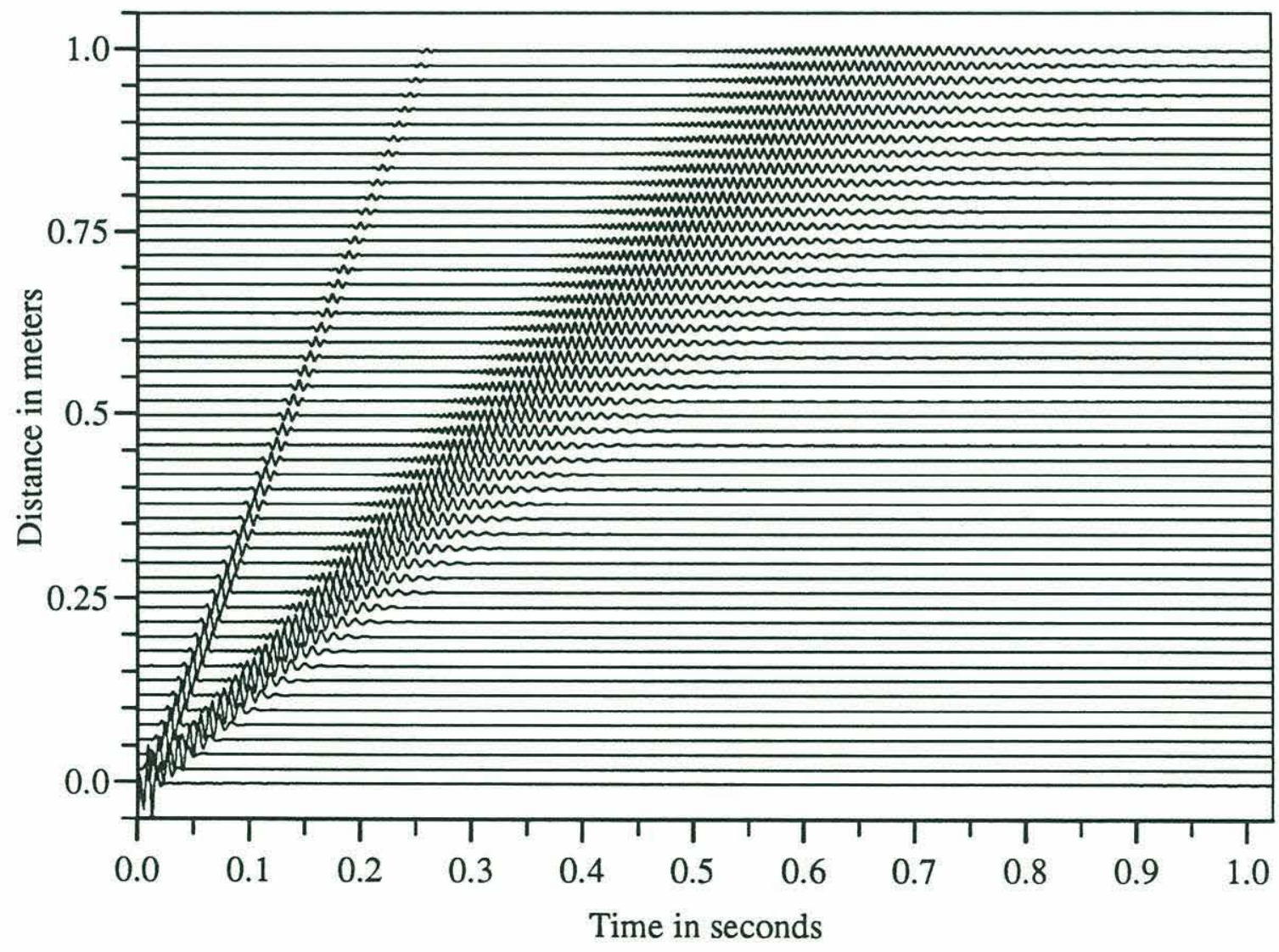

Figure 4-5: Example 1 of transmission line model.

Example 2, shown in Fig. 4-6, shows how an impedance is handled by the trans- 
mission line model. Two sources are located as in example 1. In this case, the second wavetype also has a constant group delay over frequency of $c_{g}=1 \mathrm{~m} / \mathrm{sec}$. There is no decay for either wavetype. A single impedance is located at a location of $0.5 \mathrm{~m}$ on the transmission line. For this impedance, all coefficients are set equal, $R_{00}=0.5$, $R_{01}=0.5, \ldots, T_{11}=0.5$. All waves run off the transmission line without artifact.

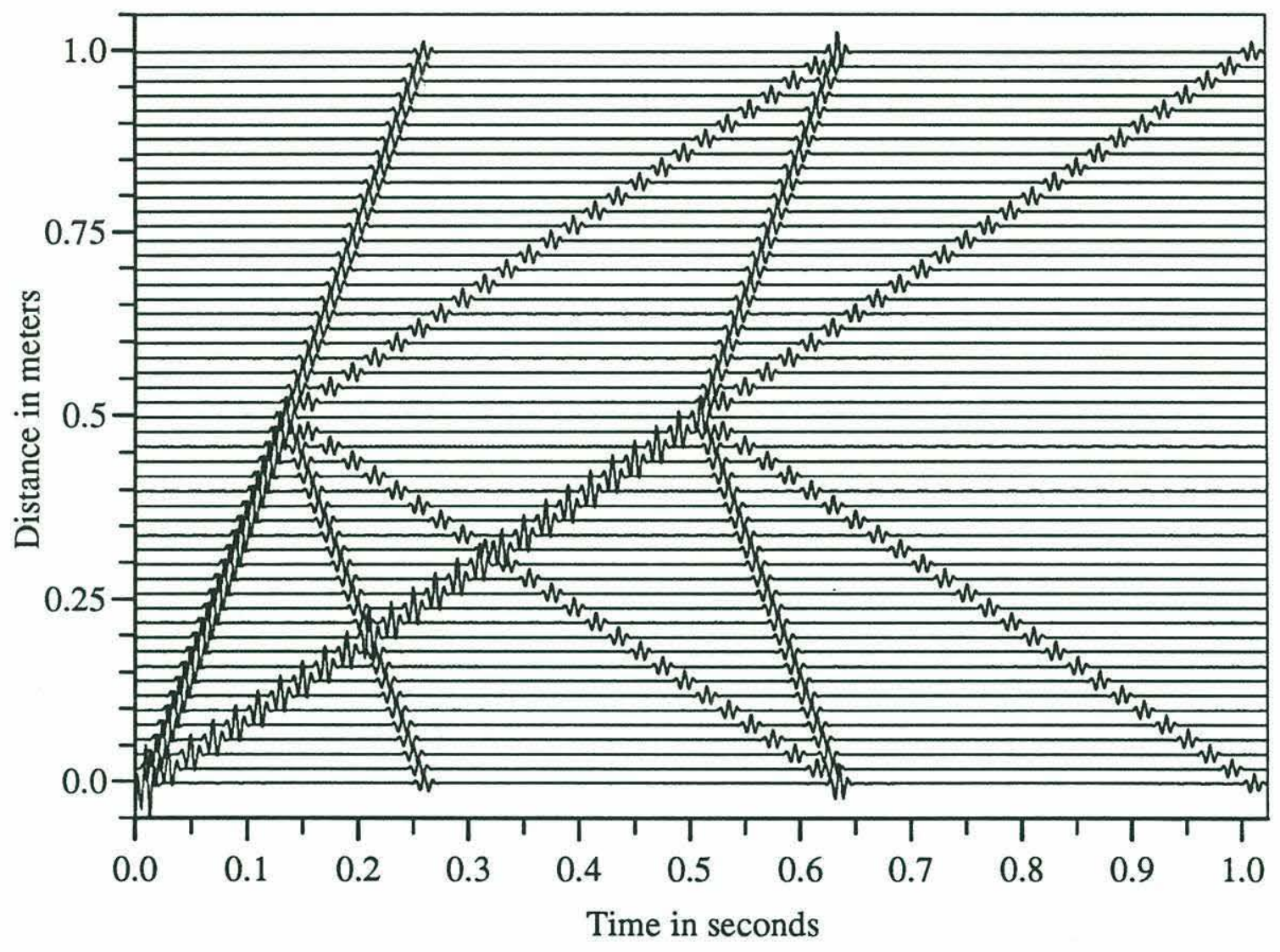

Figure 4-6: Example 2 of transmission line model.

Example 3, shown in Fig. 4-7, is identical to example 2 except the second wavetype is not allowed to "radiate." The wave is propagated, but not added to the output. Notice that when this wave couples to the first wavetype, this shows up in the output.

Example 4, shown in Fig. 4-8, illustrates the effect of slow zones on the transmission line. These are places on the line that affect the speed of traveling wavetypes. Two sources are fired as before, both wavetypes have a constant group delay over 


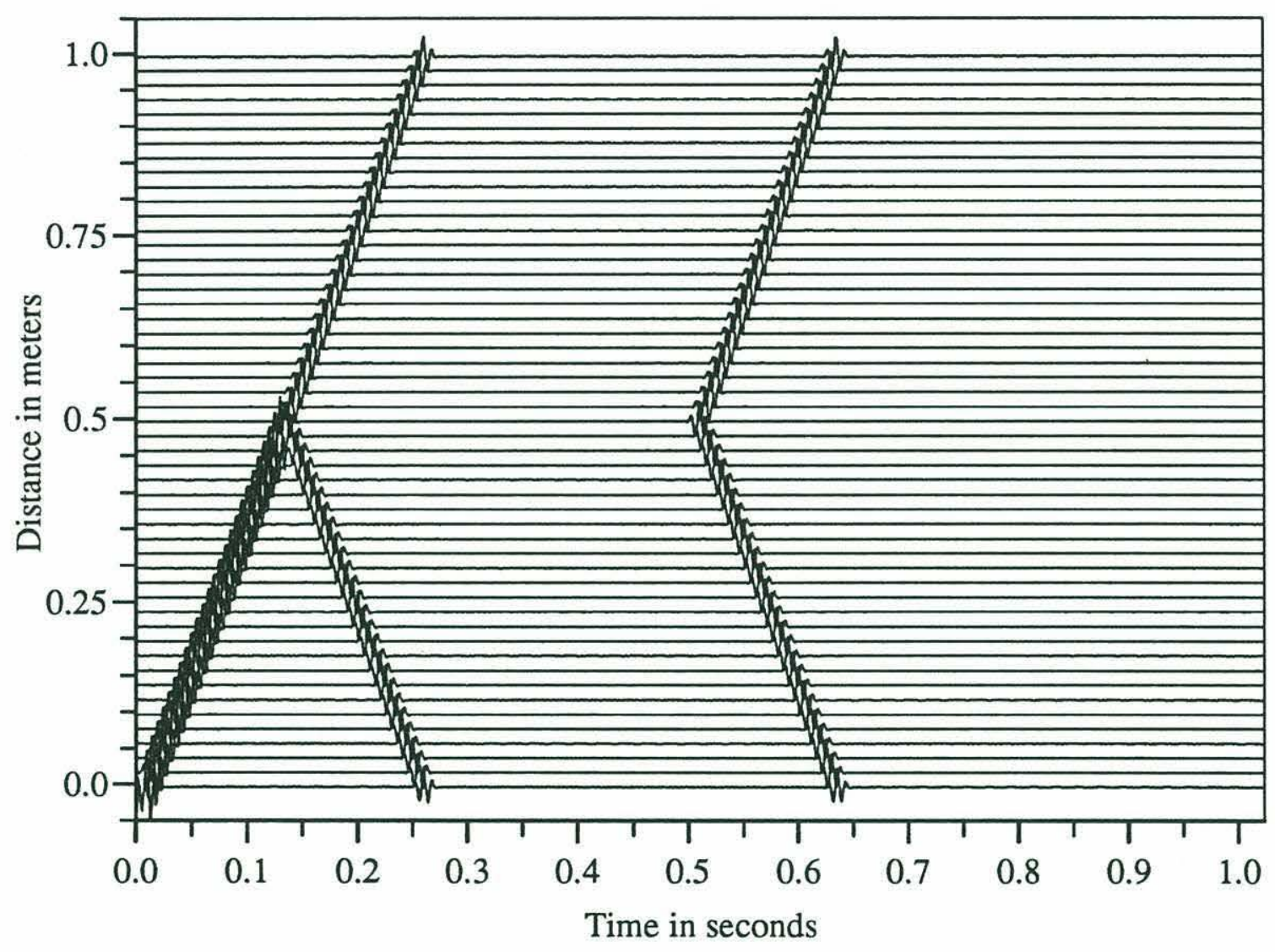

Figure 4-7: Example 3 of transmission line model. 
frequency, the first at $c_{g}=4 \mathrm{~m} / \mathrm{sec}$ and the second at $c_{g}=2 \mathrm{~m} / \mathrm{sec}$. There is no decay for either wavetype, but the second wavetype occupies only half the bandwidth of the simulation. This is manifested in a wider pulse for the second wavetype. There is a slow zone located between $0.4 \mathrm{~m}$ and $0.6 \mathrm{~m}$ on the transmission line. This zone is such that the first wavetype is dispersive with a linear group delay from to $4 \mathrm{~m} / \mathrm{sec}$ to $1 \mathrm{~m} / \mathrm{sec}$ and the second wave is non-dispersive with a group delay of $0.4 \mathrm{~m} / \mathrm{sec}$. This capability is useful for the endcap areas of the shell where the flexural wavespeeds vary from those on the cylindrical part of the shell.



Figure 4-8: Example 4 of transmission line model.

This section has served as a description of the transmission line model used to simulate the waves traveling on the shells and a general introduction to its capabilities. The particular models to match the experimental data will be described in Section 5. 


\subsection{Parameter Estimation}

The transmission line models which attempt to match the complicated characteristics of the waves traveling on each of these shells have many parameters, wavespeeds at many frequencies for two wavetypes, decay rates, reflection, transmission and coupling coefficients at several frequencies for several types of discontinuity etc. The total number of parameters can be quite large, of order 20 , each with a different range of possible values. Finding the best match to the focused experimental data has been formulated into the problem of identifying those parameters which minimize the total mean square error between the model output and the focused dataset. This is equivalent to a function minimization problem.

There are several practical issues that make solution of this problem difficult. The first issue that arises is the fact that the computation required to perform an exhaustive search over the entire parameter space to find the global minimum increases geometrically with the number of parameters. Consider a discrete function of one variable, $f(x)$, where $x$ can take on any one of $N$ discrete values. If $x$ becomes a parameter vector of length $P$ and each parameter can take on $N$ discrete values, then there are a total of $N^{P}$ values to search. However, parameter spaces are not usually discrete but instead lie within some continuous range. This means that some kind of continuous search is required. Golden mean bisectioning searches [37] can find a minimum in some small number of steps depending on the accuracy required, which is on the order of the above parameter $N$ in one parameter. Even for reasonably small values this implies that exhaustive search is computationally prohibitive, $P=20, N=10 \rightarrow N^{P}=10^{20}$, at 1 computation per millisecond this still requires $3 \times 10^{6}$ years.

There are other methods that try to solve this problem using intelligent search patterns [37]. Multi-dimensional conjugate gradient methods attempt to follow the path of steepest descent to a minimum. Unfortunately, these methods require the function derivatives which must be approximated for functions not in closed form. Simplex methods create a multi-dimensional sampling structure, called a simplex. 
The simplex is $P+1$ dimensional, in $2 D$ for example it is a triangle, and samples the function at its vertices. The simplex is then expanded, contracted and reflected around in the function space, by trying new parameters for the function at the vertex which is associated with the largest function value. This eventually contracts around a minimum value of the function.

Unfortunately, these intelligent search methods suffer from the same problem. They are often captured by local rather than global minima. For this reason, I chose to implement the parameter estimation part of the problem using a method known as simulated annealing. The method gets its name from metallurgy where liquids which are slowly cooled form more stable crystal structures. According to statistical mechanics, a thermodynamic system in thermal equilibrium has its energy distributed according to a Boltzman probability distribution:

$$
P(E)=e^{(-E / k T)}
$$

where $k$ is Boltzman's constant and $T$ is the absolute temperature. Therefore, these systems have a finite probability of moving from a lower energy state to a higher one which is dependent on the system temperature.

Simulated annealing is really descriptive of a general class of algorithms. These methods are based on the traditional methods mentioned above which are altered by allowing either the parameters or the functional values to be modified by a random variable proportional to a "temperature." This allows the basic intelligent search method to proceed generally "downhill" towards a minimum but always allow an "uphill" move proportional to temperature. If the temperature is lowered "slowly enough," it has been shown analytically that the method will arrive at the global minima. Imagine placing a ball on a tray resembling a special "egg carton" where the various depressions are of different depths with one being the deepest. The ball will roll into the closest depression, not necessarily the deepest one. This is how most methods work, they are very dependent on starting location. But now imagine shaking the tray vigorously so that the ball bounces from hole to hole. It has been 
shown analytically that if you gradually decrease the shaking, the ball will end up in the deepest hole.

Simulated annealing has been billed as the best solution to any multi-parameter estimation problem. Like any tool, it has its limitations. Each algorithm has problems some of which are unique to it and some of which are general to the method. I will outline some of the problems I encountered in solving the shell problem to indicate just where the boundaries of the method lie.

I began by using a downhill simplex based method [37] which is very widely used. I quickly discovered that one cannot use the method blindly as some might suggest. There are some caveats to this process. First, if you shake the tray too vigorously, the ball will bounce off of it completely. The parameters of your model will convergence to infinity if the initial temperature is "too high." In general, it should be chosen on the order of the error resulting from the initial parameters. Also, how slowly one decreases the shaking and by how much, i.e. the annealing schedule, is a critical component in the success of these algorithms. Selecting this schedule is more of an art than a science and is extremely problem dependent. In fact, I found that this algorithm converged prohibitively slowly for my problem. Finally, this algorithm is perfectly willing to wander around in parts of the parameter space which are not physically realizable. For instance, the flexural wavespeeds are all subsonic with respect to the water wavespeed, they do not radiate. However, when trying to solve for this parameter the algorithm often came up with estimates of the flexural wavespeed which were greater than the water wavespeed. There are no constraints which can be placed on the parameter space for this particular algorithm.

These were all motivations to move to an algorithm by Ingber. [38], known as Very Fast Simulated Re-Annealing. In this algorithm, parameter ranges are required by the program, which then performs a random walk through them. This solves the problem of physically unrealizable parameters. The algorithm samples the allowed parameter space and chooses an initial temperature for each, $T_{0 i}$, based on the values it encounters. This algorithm keeps track of a temperature independently for each parameter and uses an exponential annealing schedule. For parameter $i, T_{i}(k)=$ 
$T_{0 i} \exp \left(-c_{i} k^{1 / D}\right)$, where $D$ is the dimensionality, $k$ is the annealing time and $c_{i}$ is a constant for each parameter. Thus, the annealing schedule is automatically set and is consistent over all annealings. This algorithm converges much faster because less sensitive parameters are cooled quickly.

Moving to this algorithm was not entirely beneficial. As stated above, the program maintains temperatures for each parameter and quickly cools or fixes the values of those parameters which do not cause appreciable changes in the function to be minimized. Unfortunately, this sensitivity is often not constant over iteration. It is often dependent on starting location. For example, consider the problem of matching the temporal offset and speed of the compressional wave on the Empty shell. Until the time offset is identified, the sensitivity of the error to varying wavespeed would be fairly low. Then as the time offset estimate came close to its true value, the sensitivity of the error to wavespeed would increase dramatically. Unfortunately, the program would identify wavespeed as an insensitive parameter at the beginning of the annealing and fix it to an incorrect value. As the time offset estimate improved, enough variability of wavespeed parameter would not be available to reach its correct value and achieve the global minimum of the function. Studies by Schmidt and Baggeroer [39] have shown that these algorithms can fail even for simple problems due to adaptive use of local parameter sensitivity to adjust the search space. The most troublesome part of this particular issue is that in a large parameter space one cannot tell when this occurs, making it difficult to correct.

Another problem specific to my application is that more energetic features tend to capture the annealing. For example, in the Empty shell case, the algorithm tried to match the waveform of early arrivals very exactly by using high wavespeeds and many reflections. This is due to the fact that their energy is $10 \mathrm{~dB}$ higher than the levels later in time as shown in Fig. 3-36. As will be shown in Chapter 5, this problem could be circumvented by pre-weighting the focused data and the transmission line estimate by the inverse of the energy content in time before calculating the mean square error between them. This prevented any particular area of the data from driving the annealing. 
Finally, one must be very careful not to include periodicities in parameter spaces. The simulated annealing algorithms converge very slowly if there are multiple global minima.

All these problems led to a general suspicion of the algorithm. Early experiments, where the model was parameterized by 100 or so variables and the algorithm was allowed to search over generous spaces, did not converge to physically meaningful answers. Instead the step by step approach outlined in Chapter 5 became necessary. By parameterizing the problem tightly for just a few parameters and solving one part of the problem at a time, I was able to make progress with confidence. This allowed physically meaningful model and parameter choices at each step of the solution. In the later steps of modeling, the annealing approach had to be abandoned entirely for the global search and used only for fine tuning of parameters. Their coarse values were found through searching the space by hand, using knowledge of the problem physics as a guide. This procedure is best illustrated by the process described in of Chapter 5. It is my suggestion that anyone using any simulated annealing algorithm follow a similar procedure and keep the physics of the solution in mind. 


\section{Chapter 5}

\section{Forward Modeling Results}

This chapter shows the step-by-step process used to match the transmission line model described in Section 4.2 to the focused data for the $0^{\circ}$ incidence case. Parameters for this model vary, but are always physically meaningful. The complexity of the transmission line model is chosen to account for some part of the phenomena. Once that part of the problem is understood, its model and estimated parameters are frozen and carried along as constants to the next stage of the model complexity. This modeling effort begins with the Empty shell. Section 5.1 describes the model used to find the characteristics of the compressional waves on the Empty shell. Section 5.2 is devoted to the parameters of phenomenon induced by flexural waves in its endcaps. The flexural waves which travel on the shell itself are the subject of Section 5.3. Finally, Section 5.4 moves on to the Ribbed shell and describes the model used to find the reflection, transmission and coupling coefficients of compressional and flexural waves at the ring stiffeners. This section also includes an explanation of the resolution issue raised in Section 3.7. For the first two models, the values of the model parameters were found using the Very-Fast Simulated Re-Annealing [38] described in Section 4.3. Due to the difficulties listed in the last chapter, the more complex models had to be found by hand. 


\subsection{Compressional Waves on the Empty Shell}

The first model will attempt to match the first compressional wave that couples onto the bow endcap and propagates to the stern of the Empty shell. The real data to which the model is matched is somewhat different than that shown in Chapter 3 . The algorithms of Chapter 2 and procedures of Chapter 3 are used to create focused data at 61 samples of the shell taken from $-0.4588 m$ to $0.4588 m$ which is the length the shell would be if the cone and spherical portions of the endcap were straightened out. The middle 45 samples of this dataset represent the non-endcap region of the shell. The transmission line models the entire length to compute the timing of waves correctly. However, only these middle 45 time traces are used in the error calculation. This is done to avoid processing artifacts which arise from approximating the shell endcaps with a straight line in the focusing and endcap radiation due to diffraction.

The early time arrivals are of a very different nature than the late time arrivals on the Empty shell. The early arrivals clearly show wave packets bouncing up and down the shell. The late arrivals are comprised of slow, dispersed flexural waves recoupling into compressional waves at the endcaps after traveling the shell. Therefore, only the first $1.2 \mathrm{msecs}$ of data are used to match these first few models.

Finally, the data decays rapidly in this region. If this data were used as is, the small anomalies of the early arrivals would dominate the annealing since they are more energetic than the major features of the later arrivals. Therefore, the data is first de-weighted by the energy function integrated over space such the one shown in Fig. 3-36. The result of the data sectioning and de-weighting is shown in Fig 5-1. Notice that this section of the data looks like it should be well approximated by a transmission line. However, it is also clear that the endcap is causing some very complicated phenomena. The transmission line model cannot match all the bumps and wiggles of every process. It will however attempt to match the major features in this plot and provide a fundamental understanding of the energy traveling in the shell Note that the response before time $1.25 \mathrm{msec}$ are small processing artifacts enhanced by the de-weighting. These are not matched by the model. 


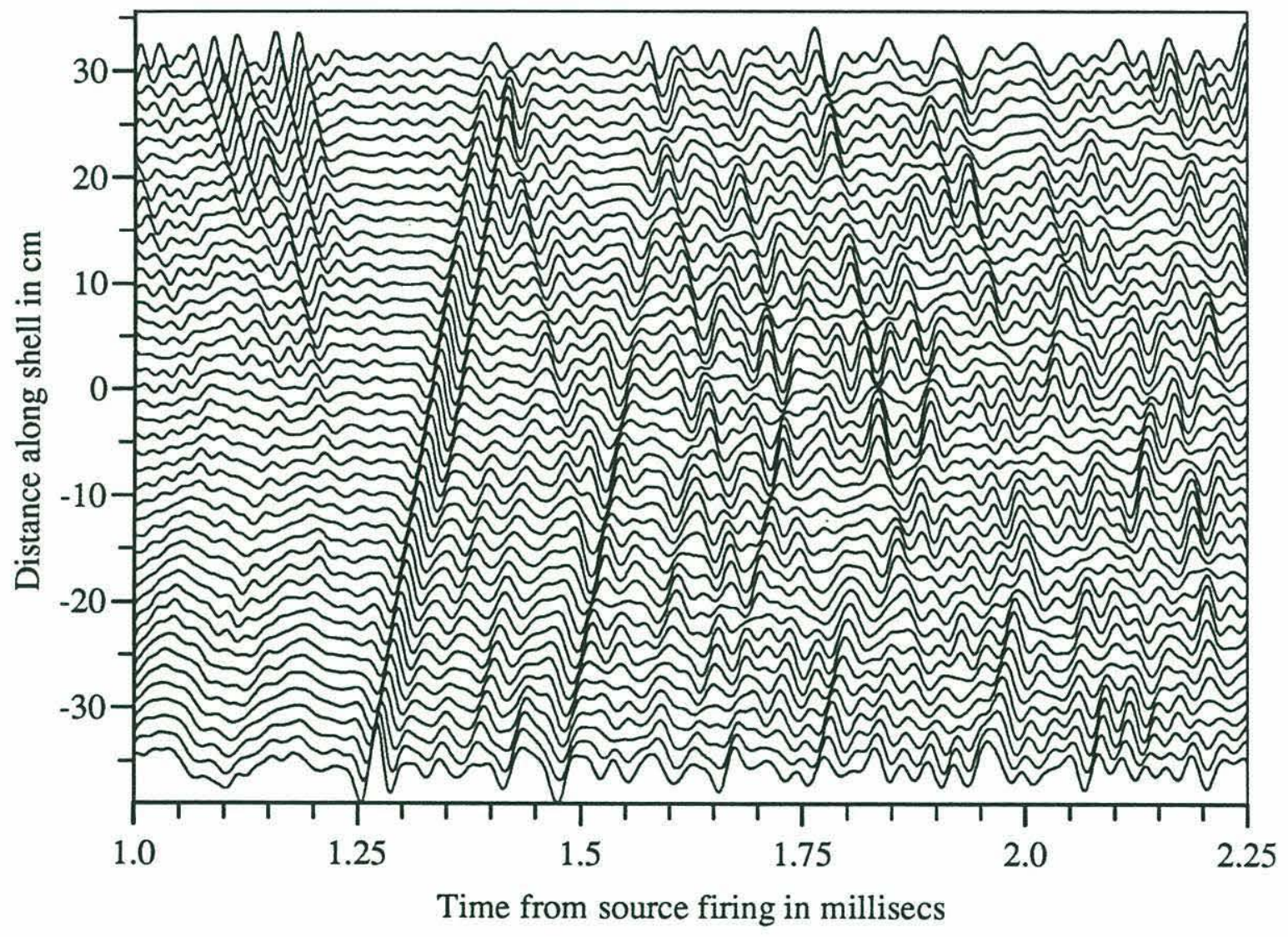

Figure 5-1: Focused Empty shell data used for match to Transmission line. Center of shell only, time traces de-weighted by average energy. 
The transmission line for this part of the Empty shell problem is shown in Fig. 5-2. The line is sampled in space at 61 points equally spaced from $-0.4588 m$ to $0.4588 m$

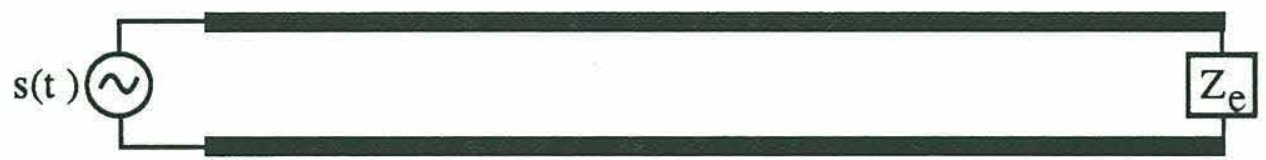

Figure 5-2: Transmission line model for first compressional wave on Empty shell.

and in time at 2048 points from $1 \mathrm{msec}$ at a sampling frequency, $f_{s}=500 \mathrm{kHz}$. The active bandwidth is from $10 \mathrm{kHz}$ to $48 \mathrm{kHz}$. As stated above the middle 45 time traces each of length 625 time samples are used to match the real data. The source $s(t)$ is a radiating wave of speed $c_{p} m / s e c$, introduced at the bow of the Empty shell transmission line model at time $t_{0} s e c$ with amplitude $a_{0} P a$ and decay rate of $\delta d B /$ msec. For this first experiment, the stern endcap is modeled by a single simple impedances, $Z_{e}$, which is a pure Transmission, $R_{c c}=0, T_{c c}=1$. This allows only one wave to propagate on the shell, the first compressional wave.

The results of this first model match to the Empty shell data is shown in Figure 53. The annealing algorithm estimates a compressional wavespeed, $c_{p} \approx 5284 \mathrm{~m} / \mathrm{s}$, a temporal offset, $t_{0} \approx 1.210$ msecs, an amplitude of $a_{0} \approx 1.528 \times 10^{-3} \mathrm{~Pa}$ and a decay rate, $\delta \approx 48.78 \mathrm{~dB} / \mathrm{msec}$. The compressional wavespeed estimate matches Corrado's[5] estimate of $5270 \mathrm{~m} / \mathrm{s}$ within a single time bin ambiguity.

The next step was to match the compressional reflection coefficient at the endcap. This is the only wavetype and coefficient that is clean enough on the shell to isolate individually, much as was the case for the Radon Transform. The model was now modified as shown in Fig. 5-4. The matched load, $Z_{e}$, was duplicated at the bow end. This is a good model for the compressional waves as it is expected that any compressional waves which propagate on the spherical portion of the endcap radiate strongly. A second kind of impedance, $Z_{c}$, was added to represent the shell-endcap discontinuities at $\pm 36.94 \mathrm{~cm}$. The source pulse was put onto the shell as before with the compressional wavespeed, decay rate, amplitude and time offset found in the last 




Figure 5-3: Transmission line model of first compressional wave on Empty shell.

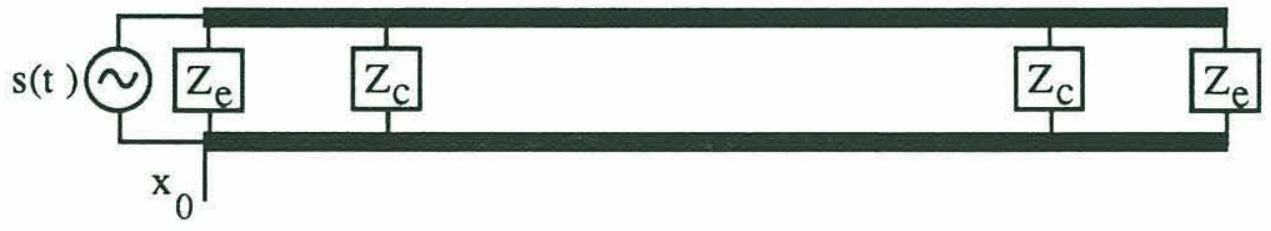

Figure 5-4: Transmission line model for reflected compressional waves on Empty shell. 
experiment set as constant. The impedances of the added discontinuities, $Z_{c}$, were identical at both ends of the shell. The compressional reflection coefficient, $R_{c c}$, at an impedance $Z_{c}$ was allowed to vary and the corresponding compressional transmission coefficient was calculated $T_{c c}=\sqrt{1-R_{c c}^{2}}$. The amplitude was modified so that the amplitude on the line after the discontinuity would match that found in the first experiment, $a_{0}^{\prime}=a_{0} / T_{c c}$.

This led to annealed values of $R_{c c}$ on the order of $R_{c c} \approx 0.01$, much lower than expected. The model was modified to allow the discontinuity location to vary. The reasoning here was that the resolution ambiguity needed to be included. The annealing found a stern discontinuity location of $39.79 \mathrm{~cm}$. This was found consistent with the actual pivot location used in the Radon Transform of Section 3.8, 39.43cm. This pivot was found by computing the location of the discontinuity required to line up the time of the first compressional wave with its reflection in the Radon Transform. Thus the shell/endcap discontinuities were located at \pm 39.79 for subsequent experiments. Using this location caused many problems in later modeling efforts. Several months of computation were wasted since the effective length of the cylinder was now longer in the model.

This assumption was re-examined. It was postulated this time that the entire shell was actually offset from center by a few centimeters. Another annealing was performed this time adding a variable offset, $x_{0}$ to all shell locations as shown in Fig. 5-4. The annealing estimated the offset to be $x_{0} \approx 2.4 \mathrm{~cm}$. This implies the stern discontinuity location of $39.35 \mathrm{~cm}$, which is consistent with the previous estimates. Comparison of the forward and backscattered arrival times show that the shell is centered in the space correctly. It is thought that the $2.4 \mathrm{~cm}$ offset is due to a $0.5^{\circ}$ bias in the receiver locations due to mechanical hysteresis in the positioning equipment.

Using this variable offset, the reflection coefficient at the discontinuity was reestimated at $R_{c c} \approx-0.34$. This is higher than the Radon Transform estimate derived in Section 3.8. However, the Radon transform estimates of the forward and backward traveling waves are really averages over the shell. These averages are susceptible to bias error if the waves decay as they travel. The decay rate of $49 \mathrm{~dB} / \mathrm{msec}$ that exists 
here due to radiation causes a decay of about half the amplitude of the wave by the time it travels to the other end of the shell. Taking this into account, a Radon Transform estimate of a reflection coefficient, $R_{c c}=-0.34$, would be underestimated at $R_{c c}^{\prime}=0.16$ which is consistent with the results of Section 3.8. The results of the spatially offset transmission line model of the bouncing compressional waves on the shell is shown in Fig. 5-5.

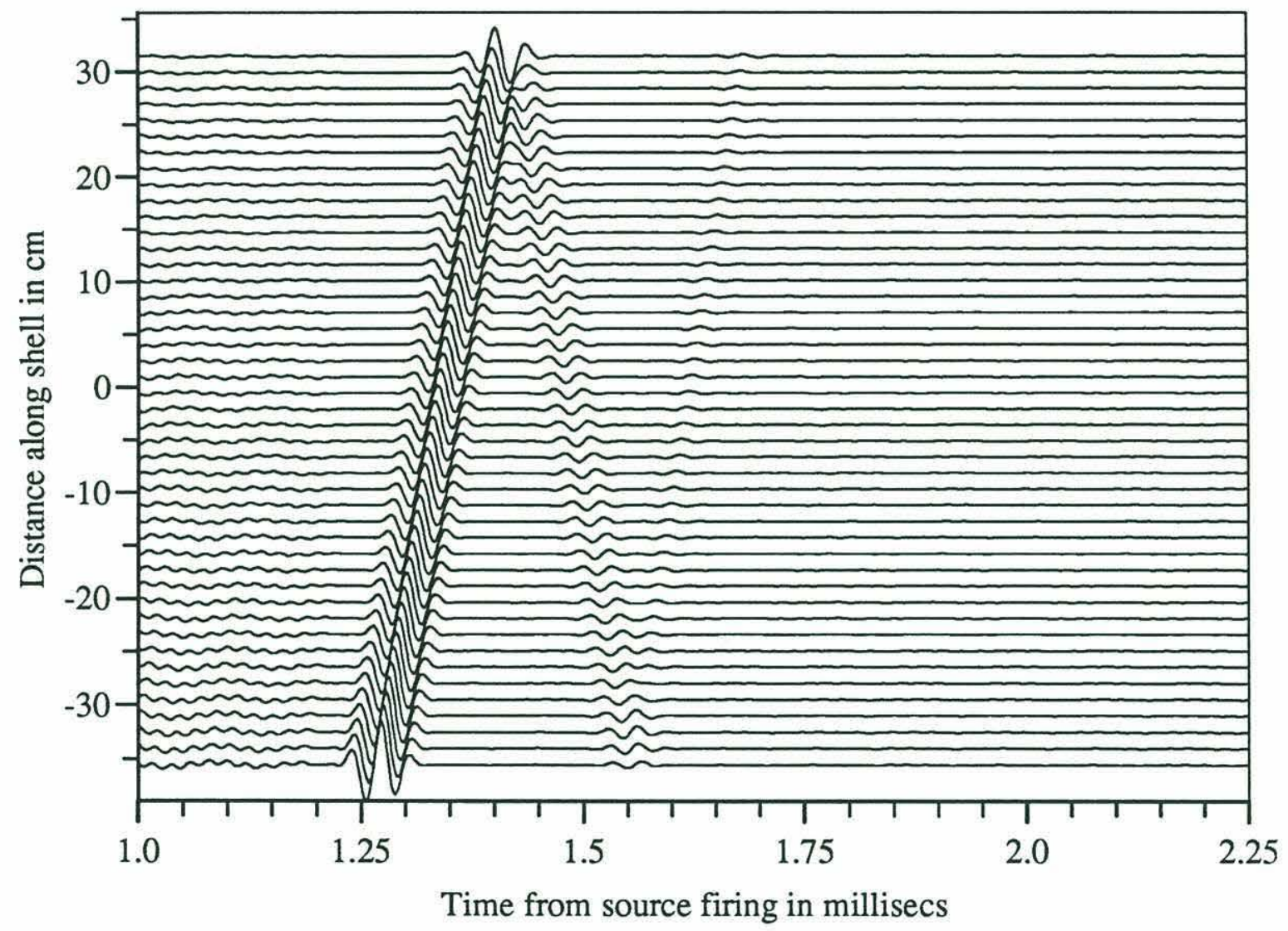

Figure 5-5: Transmission line model of reflected compressional waves on the Empty shell.

Several efforts were made to account for the second compressional return, seen in the focused data $200 \mu \mathrm{sec}$ after the first, using compressional waves only and allowing reflection at $Z_{e}$. The decay rate due to radiation for this wavetype is too high and its wavespeed too fast to allow another return of such high magnitude this long after the first return. It is not possible to construct a model which can produce the second return using compressional waves only. Slower flexural waves are required for this 
return to exist on the shell.

\subsection{Flexural Waves on the Endcaps}

In order to model the flexurally-induced components on the Empty shell, a more complicated model had to be introduced. The model of the last section was augmented by allowing another wavetype to propagate. The model flexural wavetype was not allowed to radiate directly, but was allowed to couple into compressional wavetypes at impedances. This flexural wavetype is modeled without decay, since flexural waves do not decay directly through radiation and structural damping is expected to be small.

A flexural source was placed identically in space and time with the compressional source, but its amplitude, $a_{f}$ was allowed to vary as a parameter. The bandwidth of this flexural source was defined from $10 \mathrm{kHz}$ to a parameter $f 1<48 \mathrm{kHz}$. This smaller bandwidth was allowed since some direct analysis of the second return indicates it has much lower frequency content than the first. The admittance of the shell to flexural waves seems to be higher at lower frequencies [28]. The energy in the second compressional wave seems to be contained in a fairly coherent packet, so the group speed parameter on the endcap was modeled as constant over frequency for this flexural wavetype. This is of course an approximation for what is probably a weakly dispersive wave, but seemed to work well.

The addition of a second wavetype on the transmission line meant that the reflection and transmission coefficients at each impedance became matrices defined as follows:

$$
\mathbf{R}=\left[\begin{array}{ll}
R_{c c} & R_{c f} \\
R_{f c} & R_{f f}
\end{array}\right], \mathbf{T}=\left[\begin{array}{cc}
T_{c c} & T_{c f} \\
T_{f c} & T_{f f}
\end{array}\right]
$$

where the first subscript indicates the incident wavetype, the second indicates the outgoing wavetype and $c$ and $f$ indicate compressional and flexural respectively.

The physical shell model was also augmented as shown in Fig. 5-6. The model which seemed to capture all the phenomenology was an endcap consisting of three 


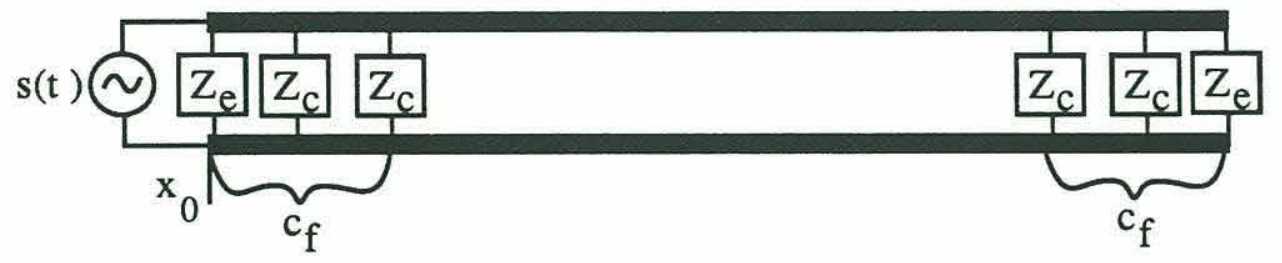

Figure 5-6: Transmission line model for early-time flexural-induced compressional waves on the Empty shell.

impedances. The end of the shell was modeled by a pure transmittance for compressional waves, due to their high radiation at the spherical section as discussed in the last section. Flexural waves were assumed to travel around the sphere and back thus modifying their amplitude by a -1 factor. Thus, for the endcaps impedance $Z_{e}$, located at $\pm 45.88 \mathrm{~cm}$, the impedance matrices are:

$$
Z_{e}: \mathbf{R}=\left[\begin{array}{cc}
0 & 0 \\
0 & -1
\end{array}\right], \mathbf{T}=\left[\begin{array}{ll}
1 & 0 \\
0 & 0
\end{array}\right]
$$

The sphere-cone and cone-cylinder discontinuities were modeled as identical impedances, since both intersections have similar angles. The model included an impedance, $Z_{c}$, at the location of each slope discontinuity, $\pm 36.94 \mathrm{~cm}$ and $\pm 42.23 \mathrm{~cm}$. All coefficients of this impedance type were allowed to vary. All locations on this model were offset by the nominal $2.4 \mathrm{~cm}$ as discussed in the last section.

The result of the hand matching of this model to the data in Fig. 5-1 is shown in Fig. 5-7. The parameters which cause this fairly good match are as follows. The flexural waves coupled onto the shell from the water and modeled by the flexural source occupy about $3 / 4$ the bandwidth of the coupled compressional wave, $f 1 \approx$ $38 \mathrm{kHz}$. Again, this is due to endcap's higher admittance to low frequency flexural waves. The group speed of the flexural waves in the endcap is $c_{f} \approx 450 \mathrm{~m} / \mathrm{sec}$, an approximate model for a weakly dispersive system, probably an apparent effect due to the reduced bandwidth. The amplitudes of the compressional and flexural waves which couple onto the shell are comparable, $a_{c} \approx 2.9 \times 10^{-3} \mathrm{~Pa}$ and $a_{f} \approx 2.2 \times 10^{-3} \mathrm{~Pa}$. Recall that this only accounts for radiated energy. Finally the coupling matrices at 


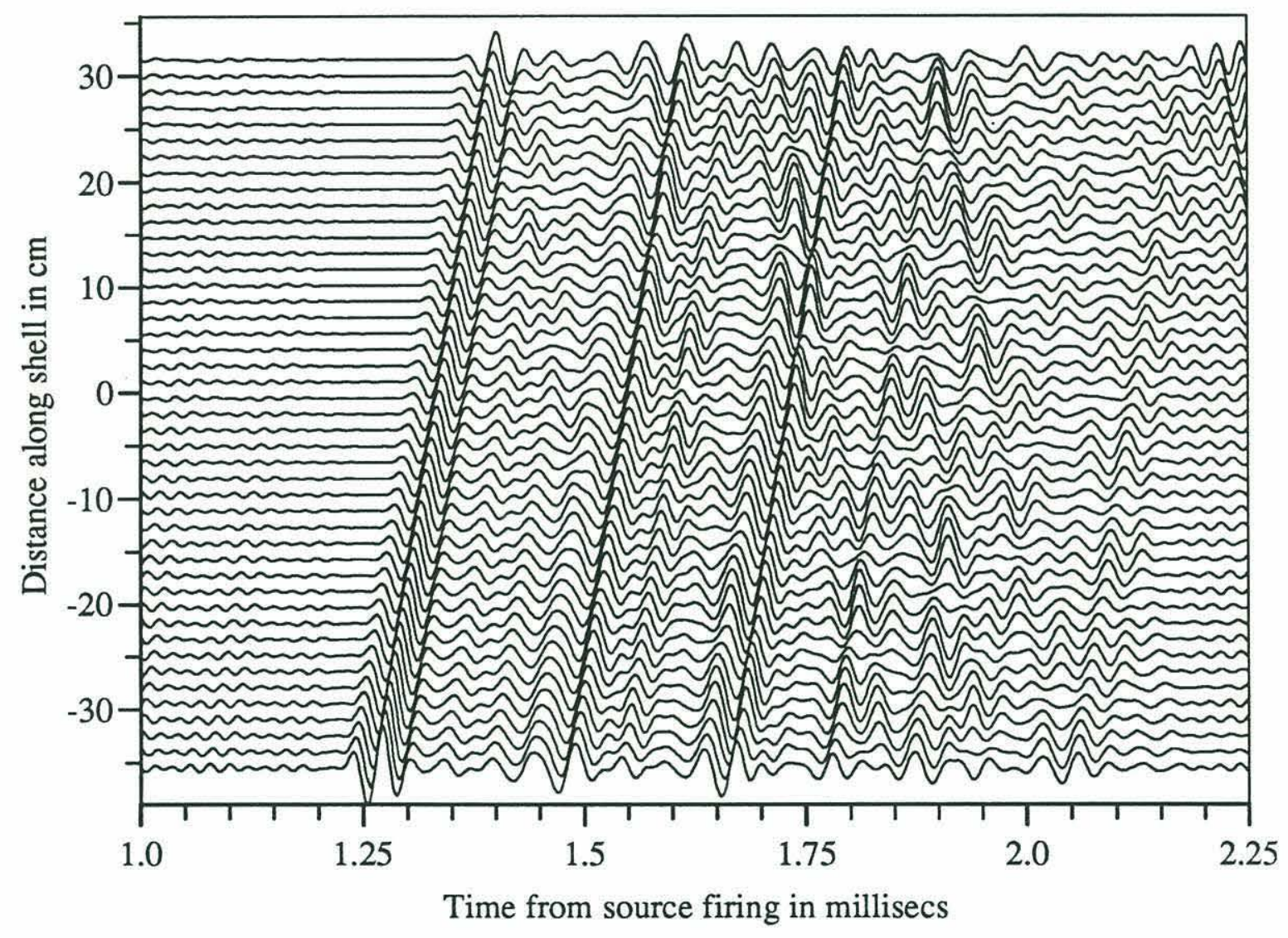

Figure 5-7: Transmission line model of early time returns on Empty shell. 
the slope discontinuities are found:

$$
Z_{c}: \mathbf{R} \approx\left[\begin{array}{cc}
-0.34 & -0.4 \\
0.2 & 0.2
\end{array}\right], \mathbf{T} \approx\left[\begin{array}{cc}
0.75 & -0.4 \\
0.4 & 0.87
\end{array}\right]
$$

Thus, waves which encounter a slope discontinuity are mainly transmitted. However, the small cross terms do lead to the complicated structure shown in Fig. 5-1 and are the main reason for continued radiation as time progresses.

This model ignored the wavespeeds of the flexural waves that travel on the cylinder section since they are expected to leave the time window considered in this part of the problem. Now that the energy in the early time arrivals has been accounted for, the energy content of the late time arrivals can be addressed.

\subsection{Flexural Waves on the Empty Shell}

The late time arrivals on the Empty shell are caused by flexural waves which have traveled the length of the shell from one endcap and coupled at the other into radiating compressional waves. The previous section showed that the slope discontinuities in the endcaps have non-zero cross-coupling terms. Thus, the flexural waves on the cylinder could have come from either flexural or compressional waves in the endcap coupling into flexural at the discontinuities. The flexural waves on the shell are modeled as dispersive waves since the late time arrivals seem to be smeared out over time. The model used for this data matching is the model found in the last section with all its parameters set to their best values.

The most satisfying aspect of this process is that no further sources are required. The energy that the last experiment allocated to flexural waves on the shell simply has to be appropriately group-delayed. This is illustrated in Fig. 5-8, where the only parameter which varies is the flexural group delay on the cylindrical portion of the shell.

The results of matching this model by hand are shown in Fig. 5-9. The group delay is linear over the simulation bandwidth from $300 \mathrm{~m} / \mathrm{sec}$ to $1000 \mathrm{~m} / \mathrm{sec}$. Compare this 


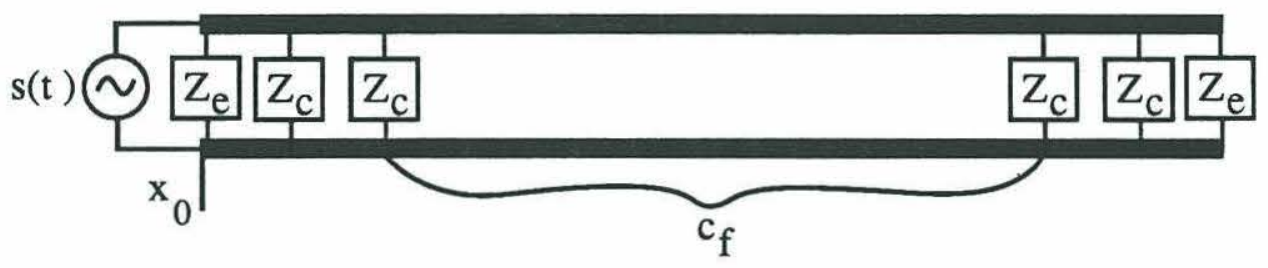

Figure 5-8: Transmission line model for late-time flexural-induced compressional waves on the Empty shell.

to Fig. 5-10, which is the corresponding focused data. Again the center 45 traces, this time 1300 time samples long to include all the late-time returns, were used for error calculation.

This representation of the late-time flexural waves created by the direct analysis was misinterpreted until the forward modeling results became available. It was assumed that the late-time energy came from a single flexural wave that had traveled the length of the shell very dispersively and coupled at the stern endcap. The transmission line model shows that even though the wave is very dispersive, it does not smear very much because most of the energy is concentrated at the center of the band for this Hamming pulse. The spreading of energy over time region $2.4-3.6 \mathrm{msec}$ is actually due to multiple arrivals of many flexural waves that have bounced around the bow endcap and then traveled the shell length. There is a great deal of flexural energy available to bounce around since the flexural waves do not decay by radiation and because the coupling coefficients to compressional waves are small. It is probably true that these waves cause the shell to ring beyond the time window that is available in these experiments.

Thus, it has been shown that the late time arrivals are due to the flexural wave energy on the shell. Even though these waves do not themselves radiate and have small coupling coefficients at discontinuities, their energy cannot be neglected as it often is for infinite cylinder analysis. They serve as an energy storage mechanism on the shell which is significant because they couple to radiating compressional wavetypes at all discontinuities. This is even more significantly proved on the Ribbed shell. 




Figure 5-9: Transmission line model of early and late time returns on the Empty shell. 


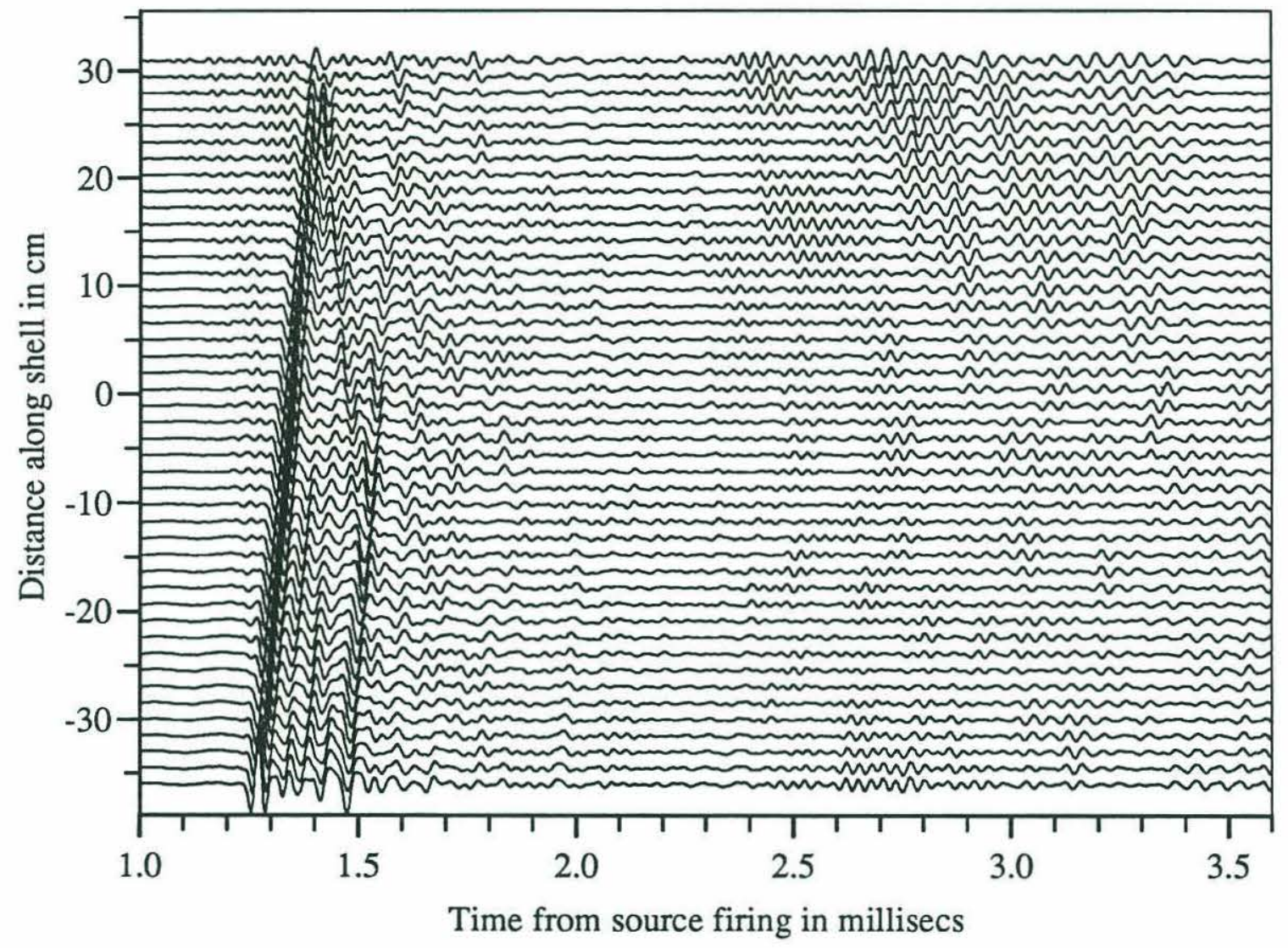

Figure 5-10: Focused Empty shell data used for match to Transmission line. Center of shell only. 


\subsection{Ring Stiffeners on the Ribbed Shell}

The Ribbed Shell presents an apparent problem for the transmission line modeling. Each rib diffracts some structural energy into the acoustic field. This is not modeled at all by the transmission line and was originally thought to be an extreme weakness of the model given the energy plots of Section 3.7 which show the large peaks in the vicinity of the rib stiffeners. However, it will be shown that these peaks are actually well accounted for by the transmission line model described here.

The Ribbed shell is to first order based on the Empty shell. The transmission line model for this shell will therefore use the transmission line model for the Empty shell and all the parameters calculated up to this point. The same model used in Section 5.3 , complete with dispersive flexural waves on the shell, is augmented by impedances representing the ring stiffeners. Although, it is thought that the coefficients for these are frequency dependent, a first order model will assume them constant over frequency and identical for all stiffeners. This model is shown in Fig. 5-11.

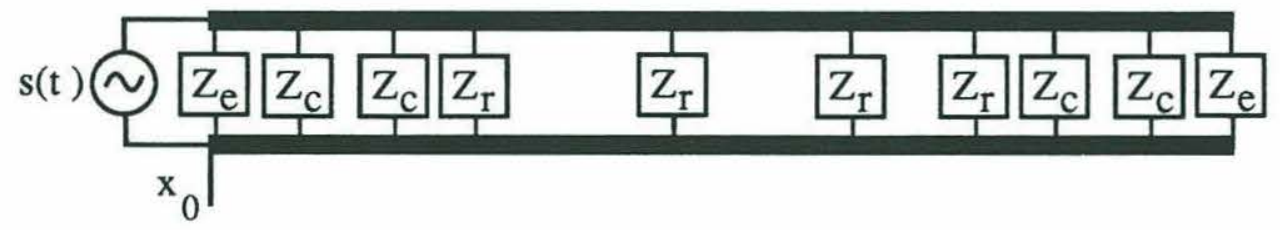

Figure 5-11: Transmission line model for the Ribbed shell.

The same sources and offsets are used for this model as for the Empty shell model. Since the reference pulses have been deconvolved from the data, no source adjustment should be necessary. The data to which the model must match is shown in Fig. 5-12. Again only the middle 45 of 61 spatial locations are used to exclude endcap diffraction effects. The time window used to clean the target-dependent tank returns for this dataset was somewhat shorter than that for the Empty shell, so only 1100 time bins can be used.

As before, the beauty of this experiment is that the hard work is already done by the previous experiments. The only variables in this experiment are those having to 


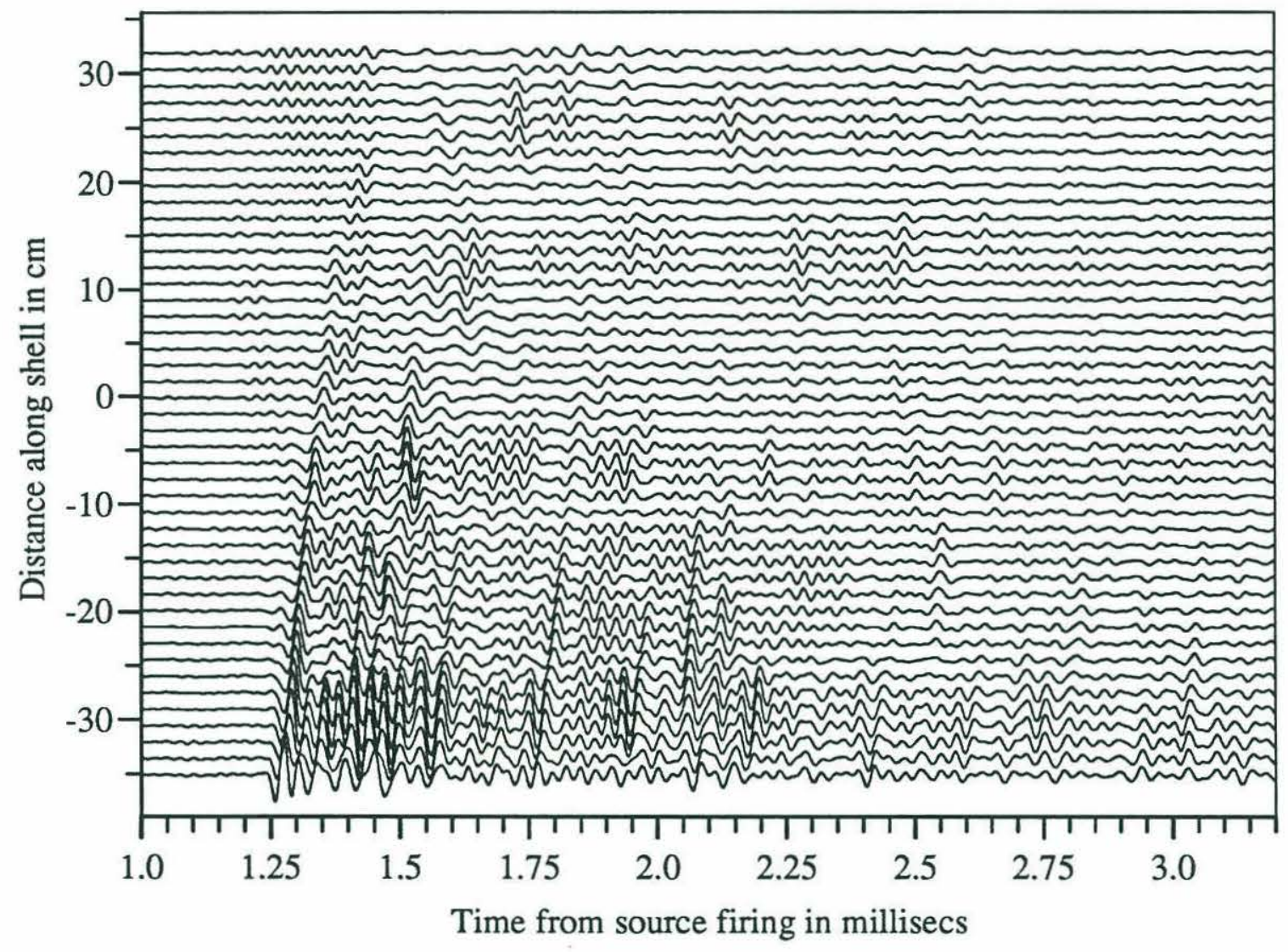

Figure 5-12: Focused Ribbed shell data used for match to Transmission line. Center of shell only. 
do with wave coupling, reflection and transmission at the ring stiffeners. The result of hand modeling is shown in Fig. 5-13. The coupling matrices at all stiffeners are

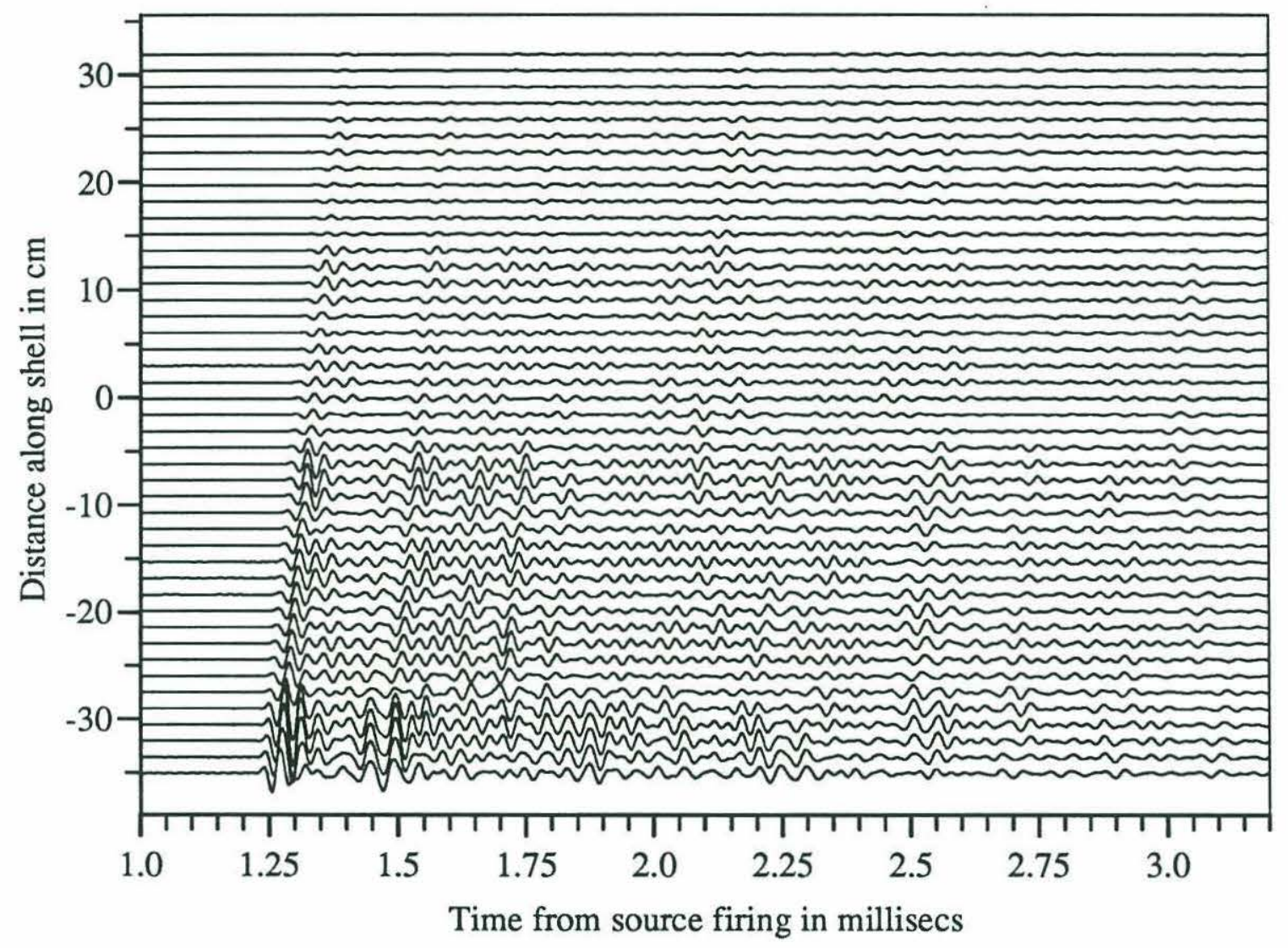

Figure 5-13: Transmission line model of the Ribbed shell.

identical in this model and are found to have the values:

$$
Z_{r}: \mathbf{R} \approx\left[\begin{array}{cc}
-0.75 & -0.3 \\
-0.3 & 0.75
\end{array}\right], \mathbf{T} \approx\left[\begin{array}{cc}
0.51 & 0.3 \\
0.3 & 0.51
\end{array}\right]
$$

These coefficients indicate that most of the energy impinging on a ring stiffener in a certain wavetype is turned back, a lesser amount is transmitted. These coefficients cause the energy containment in the bays. There is also some sharing between the compressional and flexural waves. These small coupling coefficients account for the increased radiation levels over time as seen in Fig 5-13.

This transmission line model was finally able to shed some light on the resolution 
mystery encountered in Section 3.7. The standard energy integrations of Section 3.6 were performed for the transmission line model data to determine if this model accounted for all the energy on the shell. Fig. 5-14 shows the energy integration over the shell for the actual data from Fig. 5-12 as the bold line and the integration for the transmission line model from Fig. 5-13 as the plain line. The lines do not exactly overlay, but the energy levels are generally comparable. This indicates that the modeling approach to this point has done a fair job accounting for the energy on the shell through fundamental physically-based explanations.

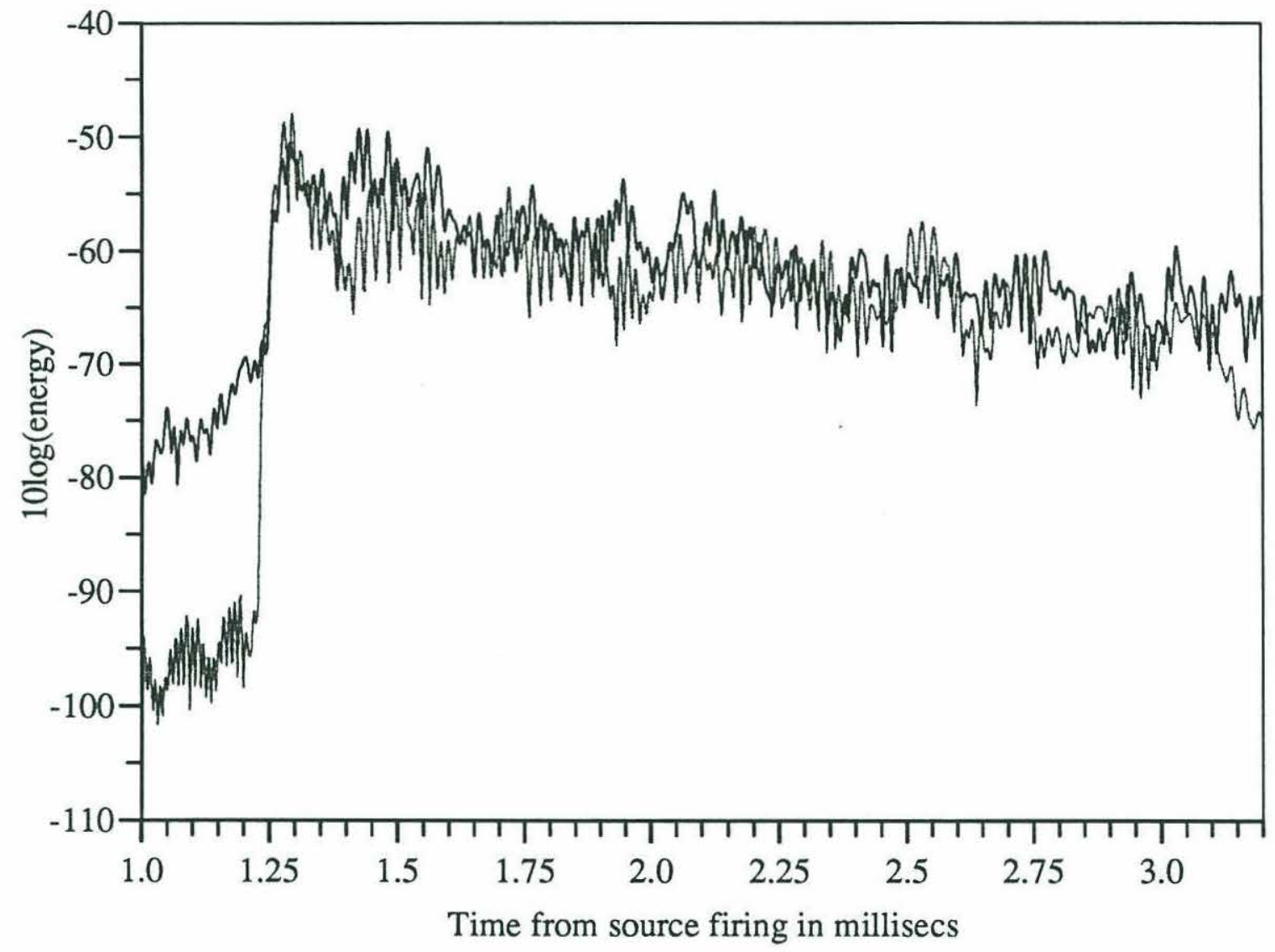

Figure 5-14: response of shell surface, energy integrated over space. Ribbed shell for $0^{\circ}$ incidence case, conventional (bold) and transmission line model (plain).

The big surprise came from the energy integration over time. Fig. 5-15 shows the energy integration over time for the actual data from Fig. 5-12 as the bold line and the integration for the transmission line model from Fig. 5-13 as the plain line. Notice that these curves line up fairly well, including the peaks near the stiffener locations. 
However, recall that the transmission line model does not include diffraction from any discontinuities. These peaks are therefore not due to that process. On further investigation, it is found that these peaks are due to pressure doubling at very rigid impedances. The reflected wave is constructively interfering with the incoming wave. This peak is therefore not a good estimate for the resolution of the processing as its width is only a function of the pulse width and compressional wavespeed. The CRB derived in Section 3.7 is probably a much better resolution estimate. This result is also consistent with the expectation that diffraction from the ring stiffener should be a weak process.

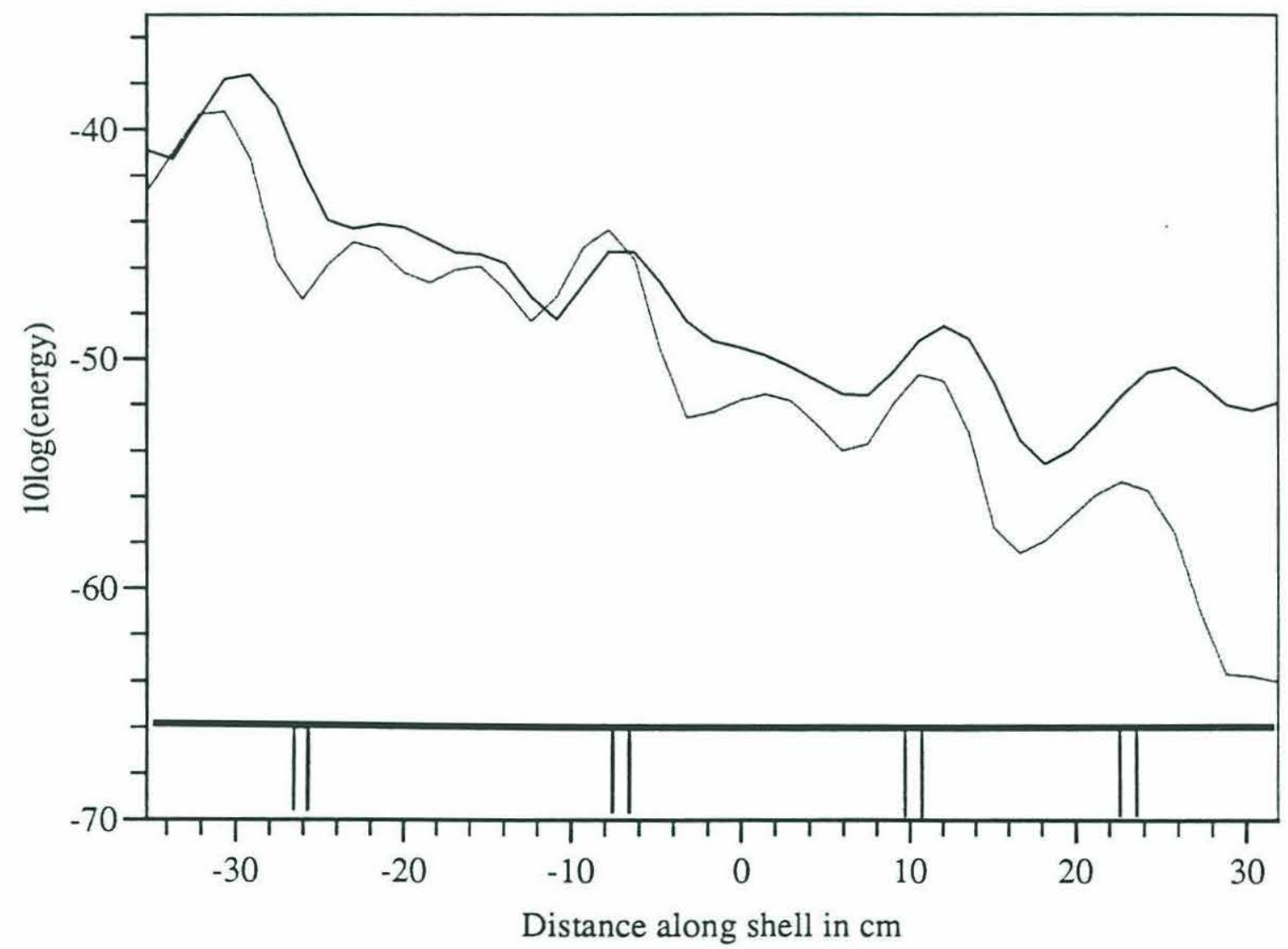

Figure 5-15: response of shell surface, energy integrated over time. Ribbed shell for $0^{\circ}$ incidence case, conventional (bold) and transmission line model (plain).

This concludes the forward modeling effort for this set of experimental data. It has been shown that direct analysis alone did not provide a sufficiently understandable picture of the fundamental physical processes at work on the shell. Only the 
understanding gained by varying the parameters of the transmission line model by hand was able to clear up several misunderstandings caused by naive interpretation of the results of the direct analysis. This shows the true value of the forward modeling approach. 


\section{Chapter 6}

\section{Conclusion}

\subsection{Discussion of Results}

This thesis has investigated the various acoustic phenomenon associated with structural wave propagation on three cylindrical shells of various internal complexity. These shells are model representations of the primary structural components of submarine hulls and were designed to investigate the mid-frequency acoustic behavior of such structures. The transient, in-plane, bistatic scattering responses of these shells to wideband pulses of spatially plane waves from various angles of incidence were collected at NRL by a synthetic array. This series of data motivated all the processing contained in this thesis.

The raw data resulting from those experiments was indecipherable due to the collection geometry, the excitation pulse used and complexity of the shells. This thesis approached the problem of understanding this data by using a Hybrid Processing Structure which included both a direct analysis process and a forward modeling process. Both of these methodologies compute a data representation in which it is more intuitive to think about the fundamental physical processes at work on the shells. This space shows the waves traveling on the shells which make up that part of the transient, dynamic structural response of the shell surface which is capable of radiating to the far field.

The direct analysis portion of the processing transformed the collected data to the 
new representation via a long processing chain of fairly standard processing blocks. First, the geometrically scattered return was estimated from the data. This process provided a beampattern for this return which turned out to be almost identical for all three shells at any particular incident angle. This return was considered to be clutter and removed so it would not obscure the waves of interest in subsequent processing. This is a new interpretation and treatment of this geometric return. Next, deconvolution of the source pulse and an array taper over the involved section of the array were used to improve time resolution and spatial sidelobe behavior respectively. Finally, a conventional focusing technique was used to backpropagate the data to the surface of the shell.

This new representation on the shell surface reveled the differences among the different shells. It became obvious that the internal ribs tend to compartmentalize energy in the bays between stiffeners, but that the energy on the Empty shell propagates the entire length unimpeded. At $0^{\circ}$ incidence, it was shown through energy integration that the Empty shell displays an initial temporal decay of $30 \mathrm{~dB} / \mathrm{msec}$ that gives way to energy increase due to re-coupling of flexural energy at the endcaps late in time, but that the Ribbed and Complex shells show a $10 \mathrm{~dB} / \mathrm{msec}$ decay throughout mainly due to flexural re-coupling by the dense stiffener distribution. Evidence that the wave-bearing internals influence energy distribution was also present. This internal system seems to reduce the response of the shell by $3 d B$ at near-beam incidence.

Unfortunately, this direct processing is limited by the parameters of the data collection. First, the in-plane geometry of the experiment does not collect the complete acoustic field for any incident angles which induce circumferencially propagating waves on the shells. This restricts quantitative analysis to the $0^{\circ}$ incidence case only. For this case, the Cramer-Rao bounds of the geometry and processing shows that better than half-centimeter spatial resolution is possible. However, the time resolution, high compressional wavespeeds and small rib separation cause sufficient aliasing in the case of the stiffened shells to make extraction of reflection coefficients by such methods as the Radon Transform impossible. Such issues required the development 
of a forward modeling approach to complete the quantitative analysis.

The representation of the waves traveling on the shell allowed a simple transmission line model of the shell to be used to approximate wave behavior for the $0^{\circ}$ incidence case. This model was created specifically for this problem and thus has been augmented to model the multiple wavespeeds, dispersion and non-radiating wavetypes which are present on the elastic shell. This model provides an excellent physical parameterization for the physics of the shell and is computationally efficient enough to allow the many iterations required to match it to the real data. The determination of model parameters was done partially by simulated annealing and partially by hand. Both were guided by the physical constraints of wave propagation on the shell.

This transmission line model was precise enough to detect a $0.5^{\circ}$ offset in receiver position and match a previous estimate of the compressional wavespeed to within one time ambiguity bin. It was also able to identify the coupling matrices at the slope discontinuities of the shell. It was found that while waves mostly transmitted through them, they have small but important compressional-flexural cross-coupling terms which are in fact responsible for all late-time energy at this incident angle. Similarly, the model was able to show that most of the energy hitting a massive rib in a stiffened shell is reflected.

The transmission line model was extremely useful for this investigation. Through modeling it was discovered that pressure doubling in the bays between stiffeners is responsible for peaks in the time energy integration for the $0^{\circ}$ incidence case. This was erroneously attributed to diffraction from the ribs themselves by naive interpretation of direct analysis results alone. This incorrect interpretation implied a serious spatial resolution reduction which in turn led to a complete discrepancy with the Cramer-Rao Bound resolution estimate. Also, the late-time energy in the Empty shell was attributed to a single highly dispersive flexural wave. The modeling reveled that multiple, moderately dispersed waves were actually responsible. Without the modeling effort, there would have been no way to test these hypotheses and prove them one way or the other. This increase in understanding shows the true value and power of the Hybrid Processing Structure. 


\subsection{Suggestions for Further Research}

The shells investigated in this thesis are very complicated. The set of measurements described in this thesis was inadequate to fully understand all the phenomena present. The following are suggestions I have for future experimentation of this type. Some measurements should be made close enough to the shell or directly on the shell in order to resolve the ambiguities created by the "acoustically invisible" flexural waves. This data need not be as extensive as the acoustic data, but it should be sufficiently accurate to infer the coupling between wavetypes at discontinuities. My data is particularly poor at incident angles which induce helical waves on the shell, since the collection array has no circumferencial extent. This prohibits examination of higher order modes on the shell. A collection at locations around the shell circumference would have been extremely valuable for these cases. Finally, the complex endcaps introduced some particularly intractable features into the response. I would recommend that future shells be built with a simpler geometry such as a hemisphere or a flat endcap to reduce the complexity of its contribution to the scattered field.

There are some improvements that could be made to both legs of the Hybrid Processing Structure. In the direct analysis leg, the model used for focusing could be improved. In particular, the compressional waves on the shell radiate with some beampattern at very particular angles. The mainlobe of this beampattern is apparently wide, which allowed my simple, omni-directional model to work as well as it did. I suspect that the array gain could be improved if an array weighting tailored to this beampattern were used in the focusing model.

In the forward modeling leg, the simple models used for the flexural waves seemed to capture most of the important phenomenology. However, these were limited by the computation time available. The next step would be to run a more complicated model, including flexural dispersion in the endcap and independent coupling matrices for the two different slope discontinuities, to improve the amplitude of compressional waves which are induced at the stern endcap. Also, the wavespeed estimates for the late-time flexural arrivals are coarse even though the energy levels for the model 
match the data fairly well. These too could be improved with more computation.

Finally, the models matched to the Ribbed shell were cursory at best. The simple reflection coefficient model used here should at least include frequency dependence. This would allow frequency filtering by the rings which is expected and has been seen in some narrowband studies by Conti [28]. It is also my belief that there is a non-negligible phase component to the coefficients at these stiffeners due to the large mass of the rib. The model could also be augmented by an estimate which would allow modeling of the diffracted energy at the ring stiffeners. If all this were done, then a qualitative comparison to the Complex shell could be made. This comparison would have to be very simple. It is my belief that the waves traveling internal to the shell cannot be analyzed with this method because there are too many coupling coefficients which cannot be infered at all, such as the coefficients between the flexural waves and internal waves.

The transmission line model itself should be improved computationally and coupled with a graphic user interface to allow a user to vary parameters interactively and understand how they affect the fundamental physical processes he is trying to model. A simplified version of this process allowed me to finally understand some of the mysteries that the direct analysis had presented to me.

Finally, these techniques could be applied directly to the new experimental series being conducted at NRL. The new model shell includes two structural layers sandwiching a visco-elastic inner layer to dampen shear waves. It would probably be valuable to compare that data to the data presented in this thesis an any or all of the various processing stages describes here to discern the effect of the multi-layer treatment on various wave processes. 


\section{Appendix A}

\section{T-line Program Input File}

This appendix contains an example input data file for the transmission line model and an explanation of the various parameters. A typical input file for the T-line program looks like the following, all lines beginning with \# are comments:

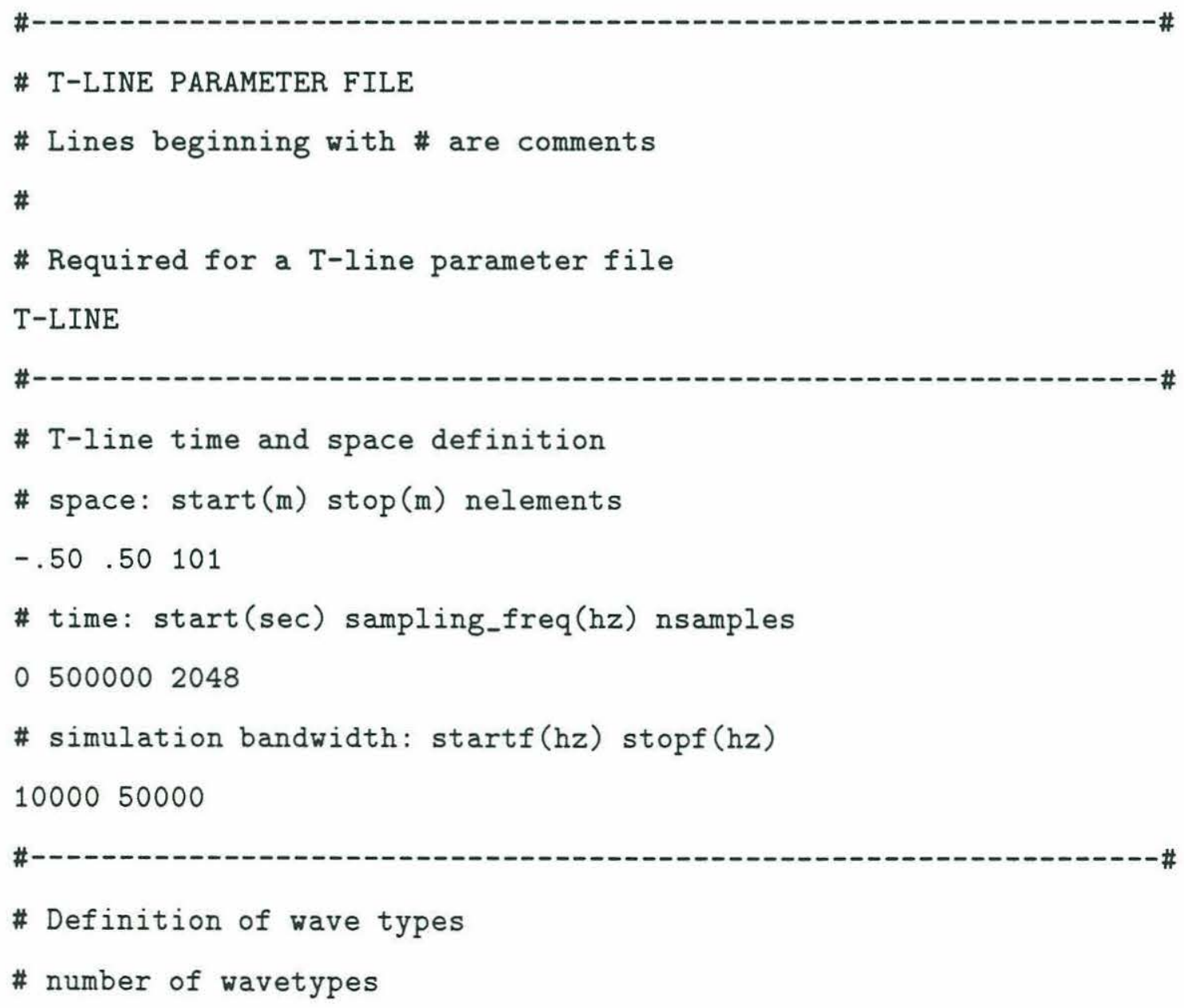


\# $\mathrm{N} \subset 1 \mathrm{c2} \ldots \mathrm{cN}$ del disp fo f1

\# $\mathrm{N}$ is order of polynomial to fit to wavespeed

\# c1-cN are wavespeeds equally spaced from startf to stopf

\# del is time decay in $\mathrm{dB} / \mathrm{sec}$

\# disp $=1$ to show wavetype $=0$ not to show wavetype

$1,5000,40000,1$

$4,200,300,400,500,1000,0$

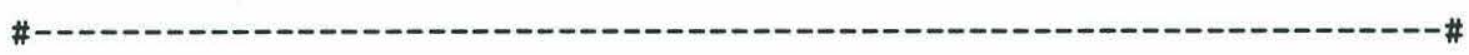

\# Source definition

\# Number of sources

2

\# Wave type location of source $x(m)$ start time $t(\mathrm{sec})$ amplitude fo f1

\# input source Hamming pulses over bandwidth

\# fO is lower bandedge of Hamming frequency window in $\mathrm{hz}$

\# $\mathrm{f} 1$ is upper bandedge of Hamming frequency window in hz

$0-0.500 .1 \quad 001 \quad 0000050000$

$\begin{array}{llllll}1 & -0.50 & 0.1 & .002 & 1000025000\end{array}$

\#---

\# Definition of discontinuity types

\# number of discontinuity types polynomial order over freq

21

\# Type 0

\# Reflection coefs ( $M \times M$ matrix where $M$ is \# of wave types)

0,0

1,0

\# Transmiss coefs

1,0

0,0

\# Type 1 
\# Reflection coefs

$0.5,0.5$

$0.5,0.5$

\# Transmiss coefs

$0.5,0.5$

$0.5,0.5$

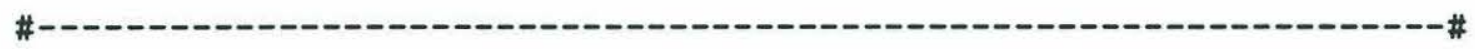

\# Discontinuity Definition

\# Number of Discontinuities

4

\# location(m) type

$-0.50$

0.50

$-0.251$

0.251

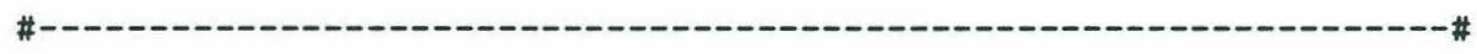

\# Optional slow zones included only if -s or - S flag is used

\# number of slow zones

2

\# $\mathrm{x} 0 \mathrm{x} 1 \quad$ ( $\mathrm{x} 0$ is where slow speed is defined.)

$-0.5-0.25$

\#slow speed: $\mathrm{N} \subset 1$ c2 $\ldots$ cN

1,4000

$4,100,200,300,400$

0.50 .25

1,4000

$4,100,200,300,400$

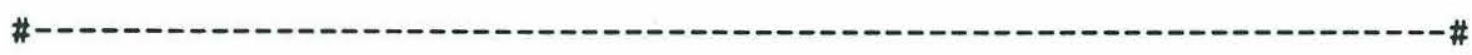

A valid input file begins with the keyword T-LINE. First, the spatial and time extent of the simulation are defined. The start and stop locations in 1D are specified, 
followed by the number of traces to be simulated. Time is specified by a start time, sampling rate and a number of samples. Finally the bandwidth of the simulation is specified in by giving the lower and upper bandedges specified in Hertz. Only frequency samples within this bandwidth will be computed.

Next, the wave types that can exist on the transmission line are defined. The number of wavetypes, $M$, is given, followed by a definition line for each wavetype. The first number, $P$, of this line is the order of the polynomial which defines the group delay over frequency, followed by samples of that curve at $P$ points equally spaced in frequency. Then the time decay parameter specified in $d B / m s e c$. Finally, either a 0 or 1 to indicate that the wavetype should not be added to the final output or that it should be added respectively.

Any number of sources can be added to the Transmission line. The number of sources is given followed by the source definition for each source. The source definition line gives the wavetype as defined in the previous section, the spatial location of the source, the time of the source firing and its peak amplitude. The source pulses are Hamming window over a frequency band which is defined for each pulse and should be contained within the frequency band of the overall simulation.

The discontinuity types are specified next. First the number of types, along with the order of the polynomial, $P$, to model the frequency response of the coefficients is provided. Next for each type is an $M \times M$ matrix for the Reflection coefficients and another for the Transmission coefficients. For each coefficient, $P$ equally spaces frequency samples must be given.

Once the discontinuity types are specified, the discontinuity locations are given. The number of discontinuities is followed by a line for each giving its location and type as defined in the previous section.

The final section is for the optional "slow zones." Any place on the transmission line can have the wavetypes travel at a different speed. The number of slow zones is given. This is followed by the extent and then a "slow speed" for each wavetype. The speeds are specified as before. 


\section{Bibliography}

[1] N. Friedman. Submarine Design and Development. Naval Institute Press, Annapolis, MD, 1984.

[2] M.C. Junger and D. Feit. Sound and Structural Interaction. MIT Press, Cambridge, MA, 1986.

[3] P. Ugincius and H. Uberall. "Creeping-wave analysis of acoustic scattering by elastic cylindrical shells." J. Acoust. Soc. Am., 43:1025-1035, 1968.

[4] P.L. Marston. "GTD for backscattering from elastic spheres and clylinders in water and the coupling of surface elastic waves with the acoustic field." J. Acoust. Soc. Am., 83:25-37, 1988.

[5] C.N. Corrado. Mid-frequency Acoustic Backscattering from Finite Cylindrical Shells and the Influence of Helical Membrane Waves. PhD thesis, Massachusetts Institute of Technology, January 1993.

[6] D.P. Mackovjac. Array Measurements of Backscattered Data from Internally Loaded Cylindrical Shells. Master's thesis, Massachusetts Institute of Technology, February 1993.

[7] E.G. Williams, H.D. Dardy, and K.B. Washburn. "Generalized nearfield acoustical holography for cylindrical geometry: Theory and experiment." J. Acoust. Soc. Am., 81:389-407, 1987.

[8] E.G. Williams, B.H. Houston, and J.A. Bucaro. "Broadband nearfield acoustical holography for vibrating cylinders." J. Acoust. Soc. Am., 86:674-679, 1989. 
[9] J. Bjarnason, J.D. Achenbach, and T. Igusa. "Acoustic radiation from a cylindrical shell with an internal plate." Wave Motion, 15:23-41, 1992.

[10] Y.P. Guo. "Normal modes propagation on a conical shell." J. Acoust. Soc. Am., submitted, 1992.

[11] D.C. Ricks and H. Schmidt. "A numerically stable global matrix method for cylindrically layered shells excited by ring forces." J. Acoust. Soc. Am., submitted, 1994.

[12] D.C. Ricks. Globally Stable Infinite Cylindrical Shell Model. PhD thesis, Massachusetts Institute of Technology, May 1994.

[13] A.V. Oppenheim and R.W. Schafer. Digital Signal Processing. Prentice-Hall, New Jersey, 1975.

[14] M. Conti and I. Dyer. "Scale model experiments on an internally loaded cylinder." Internal MIT Memorandum, June 1991.

[15] J. Capon. "High-resolution frequency-wavenumber spectrum analysis." Proc. of the IEEE, 57:1408-1418, 1969.

[16] G.V. Borgiotti and L.J. Kaplan. "Superresolution of uncorrelated interference sources by using adaptive array techniques." IEEE Trans. on Antennas and Prop., AP-27:842-845, 1979.

[17] W.F. Gabriel. "Spectral analysis and adaptive array superresolution techniques." Proc. of the IEEE, 68:654-666, 1980.

[18] A. Cantoni and L.C. Godara. "Resolving the directions of sources in a correlated field incident on an array." J. Acoust. Soc. Am., 67:1247-1255, 1980.

[19] A. Papoulis. Probability, Random Variables, and Stochastic Processes. McGrawHill Inc., New York, 1991. 
[20] D.L. Duckworth. Processing and Inversion of Arctic Ocean Refraction Data. $\mathrm{PhD}$ thesis, Joint Program in Ocean Engineering, Massachusetts Institute of Technology and Woods Hole Oceanographic Institution, September 1983.

[21] K. Hsu and A.B. Baggeroer. "Application of the maximum-likelihood method (MLM) for sonic velocity logging." Geophysics, 51(3):780-787, 1986.

[22] S.R. Deans. The Radon Transform and some of its Applications. McGraw-Hill, 1983.

[23] C.H. Chapman. "Generalized radon transforms and slant stacks." Geophys. J. R. Astr. Soc., 66:445-453, 1981.

[24] G. Turner. "Aliasing in the $\tau-p$ transform and the removal of spatially aliased coherent noise." Geophysics, 11:1496-1503, 1990.

[25] M.E. Kappus, A.J. Harding, and J.A. Orcutt. "A comparison of $\tau-p$ transform methods." Geophysics, 55:1202-1215, 1990.

[26] J. Illingworth and J. Kittler. "A survey of the hough transform." Computer Vision, Graphics and Image Processing, 44:87-116, 1988.

[27] M. Conti, Y.P. Guo, and I. Dyer. "Influence of endcaps on mid-frequency scattering from finite cylindricl shells at axial incidence." Presented at 125th Conference of the Acoust. Soc. Amer., May 1993.

[28] M. Conti and I. Dyer. "Experimental investigation of finite cylindrical shell scattering near axial incidence.: J. Acoust. Soc. Am., submitted 1994.

[29] A.B. Baggeroer, W.A. Kuperman, and H. Schmidt. "Matched field processing: Source localization in correlated noise as and optimum parameter estimation problem." J. Acoust. Soc. Am., 83(2):571-587, 1988.

[30] H. Schmidt, A.B. Baggeroer, W.A. Kuperman, and E.K. Scheer. "Environmentally tolerant beamforming for high-resolution matched field processing: Deterministic mismatch." J. Acoust. Soc. Am., 88(4):1851-1862, 1990. 
[31] H. Cox. "Sensitivity considerations in adaptive beamforming." NATO ASI on Signal Processing, pages 619-645, 1972.

[32] H. Cox. "Resolving power and sensitivity to mismatch of optimum array processors." J. Acoust. Soc. Am., 54:771-785, 1973.

[33] J.R. Fricke and A.B. Baggeroer. "Modal-slowness analysis of plate vibrations." J. Acoust. Soc. Am., 92:3228-3238, 1992.

[34] M.D. Collins, W.A. Kuperman, and H. Schmidt. "Nonlinear inversion for oceanbottom properties." J. Acoust. Soc. Am., 92(5):2770-2783, 1992.

[35] M. Dietrich. "Modeling of marine seismic profiles in $t-x$ and $\tau-p$." Geophysics, 53(4):453-465, 1988.

[36] B.L. Kennett. "Reflections, rays and reverberations." Bul. of the Seismolog. Soc. of Amer., 64:1685-1696, 1974.

[37] W.H. Press, S.A. Teukolsky, W.T. Vetterling, and B.P. Flannery. Numerical Recipies in C. Cambridge University Press, Cambridge, 1992.

[38] A.L. Ingber. "Very fast simulated re-annealing." J. Mathl. Comput. Modeling, 12:967-973, 1989.

[39] H. Schmidt and A.B. Baggeroer. "Physics-imposed resolution and robustness issues in seismo-acoustic parameter inversion." J. Acoust Soc. Am., submitted, 1994. 